\title{
Inference of Field-Sensitive Reachability and Cyclicity
}

DAMIANO ZANARDINI, Technical University of Madrid (UPM), Spain

and SAMIR GENAIM, Complutense University of Madrid (UCM), Spain

In heap-based languages, knowing that a variable $\mathrm{x}$ points to an acyclic data structure is useful for analyzing termination: this information guarantees that the depth of the data structure to which $\mathrm{x}$ points is greater than the depth of the structure pointed to by $\mathrm{x} . \mathrm{fld}$, and allows bounding the number of iterations of a loop which traverses the data structure on fld.

In general, proving termination needs acyclicity, unless program-specific or non-automated reasoning is performed. However, recent work could prove that certain loops terminate even without inferring acyclicity, because they traverse data structures "acyclically". Consider a double-linked list: if it is possible to demonstrate that every cycle involves both the "next" and the "prev" field, then a traversal on "next" terminates since no cycle will be traversed completely.

This paper develops a static analysis inferring field-sensitive reachability and cyclicity information, which is more general than existing approaches. Propositional formulæ are computed, which describe which fields may or may not be traversed by paths in the heap. Consider a tree with edges "left" and "right" to the left and right sub-trees, and "parent" to the parent node: termination of a loop traversing leaf-up cannot be guaranteed by state-of-the-art analyses. Instead, propositional formulæ computed by this analysis indicate that cycles must traverse "parent" and at least one between "left" and "right": termination is guaranteed as no cycle is traversed completely.

This paper defines the necessary abstract domains and builds an abstract semantics on them. A prototypical implementation provides the expected result on relevant examples.

Categories and Subject Descriptors: D.2.4 [Software/Program Verification]: Formal methods; F.3.1 [Specifying and Verifying and Reasoning about Programs]: Logics of programs; Mechanical verification; F.3.2 [Semantics of Programming Languages]: Program analysis; F.4.1 [Mathematical Logic]: Computational logic; I.2.2 [Automatic Programming]: Program verification

General Terms: Theory, Analysis, Verification

Additional Key Words and Phrases: Heap manipulation, Cyclicity analysis, Termination analysis, Pointer analysis, Shape analysis, Static analysis, Abstract Interpretation, Data Structures

ACM Reference Format:

Damiano Zanardini and Samir Genaim. 2014. Inference of Field-Sensitive Reachability and Cyclicity. ACM Trans. Comput. Logic V, N, Article A (January YYYY), 39 pages.

DOI :http://dx.doi.org/10.1145/0000000.0000000

\section{INTRODUCTION}

Programming languages with dynamic memory allocation, such as Java, allow creating and manipulating linked data structures in the heap. The presence of cyclic data structures in the heap is a challenging issue in the context of termination analy-

Authors' addresses: Damiano Zanardini, Departamento de Inteligencia Artificial, Escuela Técnica Superior de Ingenieros Informáticos, Campus de Montegancedo, Boadilla del Monte, 28660 Madrid, Spain; Samir Genaim, Departamento de Sistemas Informáticos y Computación, Facultad de Informática, Universidad Complutense de Madrid, C/ Profesor José García Santesmases s/n, 28040, Madrid, Spain.

Permission to make digital or hard copies of part or all of this work for personal or classroom use is granted without fee provided that copies are not made or distributed for profit or commercial advantage and that copies show this notice on the first page or initial screen of a display along with the full citation. Copyrights for components of this work owned by others than ACM must be honored. Abstracting with credit is permitted. To copy otherwise, to republish, to post on servers, to redistribute to lists, or to use any component of this work in other works requires prior specific permission and/or a fee. Permissions may be requested from Publications Dept., ACM, Inc., 2 Penn Plaza, Suite 701, New York, NY 10121-0701 USA, fax +1 (212) 869-0481, or permissions@acm.org.

(C) YYYY ACM 1529-3785/YYYY/01-ARTA $\$ 15.00$

DOI: http://dx.doi.org/10.1145/0000000.0000000 
sis [Berdine et al. 2006, Cook et al. 2006; Albert et al. 2008: Spoto et al. 2010], resource usage analysis [Wegbreit 1975; Debray and Lin 1993; Albert et al. 2012: Albert et al. 2013], garbage collection |Jones and Lins 1996], etc. Consider the loop "while (x!=null) do $x:=x . f$ ": if $x$ points to an acyclic data structure before the loop, then the depth of the data structure to which $x$ points strictly decreases after each iteration; therefore, the number of iterations is bounded by the initial depth of the structure. On the other hand, in general, nothing can be said about such a decrement if acyclicity cannot be demonstrated, unless more complex, program-specific or non-automated reasoning is performed. This makes acyclicity information essential in order to bound loop iterations and, by extension, prove termination.

In mainstream Object-Oriented programming languages, data structures are usually modified by means of field updates. Consider $\mathrm{x} . \mathrm{f}:=\mathrm{y}$ : if $\mathrm{x}$ and $\mathrm{y}$ are guaranteed to point to disjoint parts of the heap before the command, then there is no possibility to create a cycle. On the other hand, if they are not disjoint, i.e., they share a common part of the heap, then a cyclic structure might be created. This simple mechanism, denoted in the following as $\mathcal{Y}_{\text {sh }}$, has been used in previous work [Rossignoli and Spoto 2006]: $x$ and $y$ are declared as possibly cyclic whenever they share before the update. Refinements of $\boldsymbol{Y}_{\text {sh }}$ have been proposed [Ghiya and Hendren 1996; Genaim and Zanardini 2010; Genaim and Zanardini 2013: Nikolic and Spoto 2014], which also consider the reachability between program variables. In this example, the acyclicity information can be more precise if it is possible to know how $\mathrm{x}$ and $\mathrm{y}$ share: in general, it can be the case that (1) $\mathrm{x}$ and $\mathrm{y}$ alias, i.e., point directly to the same location; (2) $\mathrm{x}$ reaches the location pointed to by $\mathrm{y}$; (3) y reaches the location pointed to by $x$; or (4) they both indirectly reach a common location (here, this case is referred to as deep sharing, see Section 4.1.1). The field update $\mathrm{x} . \mathrm{f}:=\mathrm{y}$ might create a cycle only in cases (1) or (3). The latter approach is able to prove acyclicity in cases like "y:=x.next.next; $x$.next:=y;" (which typically removes an element from a linked list), where the former fails. For simplicity, this technique will be denoted by $\boldsymbol{y}_{r c}$, ignoring discrepancies between the different works implementing such a reachability-based analysis. $\boldsymbol{y}_{r c}$ improves on $\boldsymbol{y}_{s h}$ in that the class of data structures which can be proved to be acyclic is larger.

However, recent research [Scapin 2012: Brockschmidt et al. 2012] went one step ahead by proving, in some cases, the termination of programs even if the data structures they traverse 1 are cyclic. In fact, cycles often enjoy certain properties which allow to guarantee that loops never traverse them completely. Suppose that $x$ points to a cyclic data structure, and the loop while ( $x !=$ null) do $x:=x . f$ is supposed to traverse it. Recent works were able to prove termination if either (a) no cycle can involve f [Scapin 2012]; or (b) cycles have to involve a set $X$ of fields which contains fields different from $f$ [Brockschmidt et al. 2012]2].

The present cyclicity analysis, denoted in the following by $\mathcal{Y}_{f l d}$, is more general and more precise than the above approaches, and allows inferring field-sensitive reachability and cyclicity information which can be used to prove termination of a wider class of programs. The information inferred by $\boldsymbol{y}_{\text {fld }}$ takes the form of propositional formulx which indicate which are the fields involved (1) in paths between two variables; or (2) in cycles reachable from a variable. A propositional formula can tell that a field never occurs in cycle, or that it always occur, or that its presence in cycles is conditional. Consider the case of a Tree class implementing trees where each node has a left and a right field pointing to its left and right sub-trees, respectively, and a parent field pointing to the parent node. Suppose also that two loops traverse the tree (1) from the root to a leaf, by following a certain path; and (2) from this leaf, back to the root. The first loop traverses left and right a certain number of times, while the second only traverses parent. This kind of tree is a

\footnotetext{
${ }^{1}$ The idea of traversing fields will be defined precisely later, but can be understood as dereference.

${ }^{2}$ This paper also deals with other cases of algorithms on cyclic data structures, which are beyond the scope of this discussion.
} 
cyclic data structure; however, it enjoys the property that every cycle has to traverse parent and at least one between left and right. Condition (a) above does not hold for any of the loops, since they traverse fields which are actually involved in cycles. Condition (b) does not hold either, since the only field which is involved in all possible loops is parent, but the second loop actually traverses it, so that termination cannot be proved. On the other hand, the propositional formulæ computed by the present analysis represent the desired cyclicity information which allows proving termination of both loops, since it is possible to prove that they will never traverse a cycle completely. Another example of cyclic structure where cycles can traverse several different sets of fields is a cyclic grid, i.e., some kind of bidimensional double-linked list where each node has left, right, up, and down links to neighbour nodes. This data structure has cycles which traverse left and right, or up and down, but also longer cycles traversing all fields.

Following the well-known theory of Abstract Interpretation [Cousot and Cousot 1977], the paper introduces abstract domains representing the properties of interest, and discusses their relation with existing work. A sound abstract semantics is built on these domains, which computes the desired reachability/cyclicity information. An intra-procedural subset of the abstract semantics has been implemented, and gives the expected result on the examples discussed in this introduction.

Main contributions. The main contributions of the present paper are as follows:

- The paper defines abstract domains which capture field-sensitive reachability and cyclicity information in form of propositional formulæ.

- The domains are compared to related work and proved to be more precise.

- A sound abstract semantics is built on the abstract domains.

- The approach is partially implemented (only a subset of the intra-procedural component), and the expected result is obtained on relevant examples.

\subsection{Related work}

The present paper is very related to research in the area of Pointer analysis [Hind 2001], which considers properties of the heap and builds static analyses to enforce them. Clearly, techniques which directly deal with the reachability and cyclicity originated by paths in the heap represent the closest work in this area. Apart from that, Aliasing, Sharing, Points-to and Shape analysis are the most related pointer analyses which can be found in the literature.

Termination analysis is a well-established research area which overlaps with Pointer analysis when heap-manipulating programming languages are considered; it also has to be discussed as related work. Finally, Resource-usage analysis is also related because the same results which are useful in order to prove termination can also help in estimating the resource consumption of a program.

Pointer Analysis. A well-known technique in Pointer analysis, Aliasing analysis [Hind 2001] investigates the program variables which might point to the same heap location at runtime. Sharing analysis [Secci and Spoto 2005] is more general in that it determines if two variables $v_{1}$ and $v_{2}$ can reach a common location in the heap, i.e., if the portions of the heap which are reachable from $v_{1}$ and $v_{2}$ are not disjoint. Aliasing between two variables implies that they also share. Points-to analysis computes the set of objects which might be referred to by a pointer variable.

Research on Shape Analysis [Wilhelm et al. 2000] basically reasons about heapmanipulating programs in order to prove program properties. In most cases, safety properties are dealt with [Bardin et al. 2004: Sagiv et al. 2002: Rinetzky et al. 2005]. On the other hand, termination is a liveness property, and is, typically, the final property to be proved when analyzing cyclicity; therefore, work on liveness [Reynolds 2002: Balaban et al. 2005; Berdine et al. 2006: Cook et al. 2006; Brotherston et al. 2008 is closer to the present ap- 
proach. Most papers use techniques based on Model Checking [Müller-Olm et al. 1999], Predicate Abstraction [Graf and Saïdi 1997], Separation Logic [Reynolds 2002] or Cyclic proofs [Brotherston et al. 2008] in order to prove properties of programs manipulating the heap. Typically, shape analyses capture aliasing and points-to information, and build a representation of the heap from which reachability information can be obtained. Such analyses are very precise, sometimes at the cost of (i) limiting the shape of the data structures which can be analyzed; (ii) simplifying the programming language to be dealt with; or (iii) reducing scalability.

Reachability and Cyclicity analysis. The oldest notion of reachability dates back to [Nelson 1983]: his reachability predicate is supposed to tell if a heap location reaches another one in a linear list. A reachability-based acyclicity analysis for $\mathrm{C}$ programs was developed by [Ghiya and Hendren 1996. That analysis was presented as a data-flow analysis, and the terms "direction" and "interference", were used for, respectively, reachability and sharing. Analyses which compute basically the same information were presented in more recent work. [Genaim and Zanardini 2010: Genaim and Zanardini 2013] describe a formalization of the analysis proposed by [Ghiya and Hendren 1996] in the framework of Abstract Interpretation, based on a Java-like Object-Oriented language and provided with soundness proofs. The same analysis has been also formalized by means of Abstract Interpretation by [Nikolic and Spoto 2014], which efficiently implement it in the Julia analyzer for Java (bytecode) and Android 3 . As already discussed in the introduction, the analysis proposed by [Rossignoli and Spoto 2006] is less precise since it does not consider reachability in order to detect cycles. The present work also builds upon the results presented in [Scapin 2012; Brockschmidt et al. 2012. The relation with such works was explained in the introduction, and will be made even more clear in the rest of the paper, especially in Section 3.6

Termination and Resource-usage Analysis. The main goal of most approaches to reachability and cyclicity analysis is to help Termination analysis proving the termination of loops traversing data structures in the heap. This is the case of practically all the papers discussed in the previous paragraph. In particular, [Scapin 2012] and [Brockschmidt et al. 2012] are able to prove termination even when some kinds of cyclic data structures are traversed. Proving termination of a given loop is typically done by finding a ranking function that decreases in every iteration. For loops traversing acyclic data structures, the bound is interpreted in terms of the depth of the data structure [Spoto et al. 2010] (e.g, the length of a list, the depth of a tree, etc.). On the other hand, for cyclic data structures which are traversed in an acyclic way, the bound can be interpreted in terms of the acyclic depth, i.e., the maximal length of acyclic paths.

The abstract domains defined by [Scapin 2012] can assess that a data structure, although possibly cyclic, might only contain cycles with certain characteristics; namely, that the fields traversed by the cycle do not belong to a given set. This way, it is possible to prove that traversing a cyclic data structure will terminate, provided the traversal only concerns fields which are guaranteed not to appear in cycles. Importantly, this abstract domain is not able to deal with the examples of the cyclic tree and the double-linked list (Section 1.2 and 1.3), since the field traversed by the loop are involved in cycles. As a matter of fact, the abstract domain $\overline{\mathcal{I}}_{r c}$ used by $\mathcal{Y}_{f l d}$ and presented in Section 3 is strictly more expressive than the one used by [Scapin 2012], as proved in Section 3.6.4.

[Brockschmidt et al. 2012] address a similar problem from a similar point of view: termination can be proved in cases where it is guaranteed that any cycle must traverse some set of fields. Their work can prove the termination of a loop traversing a double-linked list by building a Termination Graph and proving properties which entail program termi-

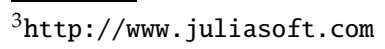


termination analyzers reject the second loop as potentially diverging, since acyclicity of the data structure pointed to by $x$ cannot be proved (indeed, it is cyclic). On the other hand, the presented approach analyzes the loop in lines 3-9 and infers that any cyclic path must traverse both $\mathrm{n}$ and $\mathrm{p}$, thus making possible to prove that the loop in lines 10-12 terminates. A similar piece of information is obtained by [Brockschmidt et al. 2012]. This example will be further discussed in Section 4.7

\section{A SIMPLE OBJECT-ORIENTED LANGUAGE}

This section defines the syntax and the denotational semantics of a simplified version of Java. Class, method, field, and variable names are taken from a set $\mathcal{X}$ of valid identifiers. A program consists of a set of classes $\mathcal{K} \subseteq \mathcal{X}$ partially ordered by the subclass relation $<$. Following Java, a class declaration takes the form "class $\kappa_{1}$ [extends $\left.\kappa_{2}\right]\left\{t_{1} f l d_{1} ; \ldots t_{n} f l d_{n} ; M_{1}\right.$ $\left.\ldots M_{k}\right\}$ " where each " $t_{i} f l d_{i}$ " declares the field $f l d_{i}$ to have type $t_{i} \in \mathcal{K} \cup\{$ int $\}$, and each $M_{j}$ is a method definition. The optional statement "extends $\kappa_{2}$ " declares $\kappa_{1}$ to be a subclass of $\kappa_{2}$. A method definition takes the form " $t m t h\left(t_{1} w_{1}, \ldots, t_{n} w_{n}\right)\left\{t_{n+1} w_{n+1} ; \ldots . t_{n+p} w_{n+p} ;\right.$ com $\}$ " where: $m t h \in \mathcal{X}$ is the method name; $t \in \mathcal{K} \cup\{$ int $\}$ is the type of the return value; $w_{1}, \ldots, w_{n} \in \mathcal{X}$ are the formal parameters; $w_{n+1}, \ldots, w_{n+p} \in \mathcal{X}$ are local variables; $t_{n+k}$ is the declared type of $w_{n+k}$, hereafter denoted by $\delta\left(w_{n+k}\right)$; and the command com follows this grammar:

$$
\begin{aligned}
\exp ::= & n \mid \text { null }|v| v \cdot f l d\left|\exp _{1} \oplus \exp _{2}\right| \text { new } \kappa \mid v \cdot m t h(\bar{v}) \\
\operatorname{com}::= & \text { skip }|v:=\exp | v \cdot f l d:=\exp \left|\operatorname{com}_{1} ; \operatorname{com}_{2}\right| \\
& \text { if } \left.\exp \text { then } \operatorname{com}_{1} \text { [else } \operatorname{com}_{2}\right] \mid \text { while } \exp \text { do com | return } \exp
\end{aligned}
$$

where $v, m t h, f l d \in \mathcal{X} ; \bar{v} \in \mathcal{X}^{*} ; n \in \mathbb{Z} ; \mathcal{K} \in \mathcal{K}$; and $\oplus$ is a binary operator on int. For simplicity, and without loss of generality, conditions in if and while statements are assumed not to have side effects. A method signature $\kappa . m t h\left(t_{1}, \ldots, t_{n}\right): t$ refers to a method $m$ th defined in class $\kappa$, taking $n$ parameters of type $t_{1}, \ldots, t_{n} \in \mathcal{K} \cup\{$ int $\}$, and returning a value of type $t$. Given a signature $\mathrm{mth}$, let $\mathrm{mth}^{b}$ be its code com (i.e., the command appearing in its definition); $\mathrm{mth}^{i}$ be its set of input variables $\left\{t h i s, w_{1}, \ldots, w_{n}\right\}$, where this refers to the object receiving the call; $\mathrm{mth}^{l}$ be its set of local variables $\left\{w_{n+1}, \ldots, w_{n+m}\right\}$; and $\mathrm{mth}^{s}=\mathrm{mth}^{i} \cup \mathrm{mth}^{l}$. Given a program, $\mathcal{F}$ denotes the set of fields declared in itf.

A type environment $\tau$ is a partial map from $\mathcal{X}$ to $\mathcal{K} \cup\{$ int $\}$ which associates types to variables at a given program point. Abusing notation, when it is clear from the context, type environments will be confused with sets of variables when types are not important; i.e., $v \in \tau$ will stand for $v \in \operatorname{dom}(\tau)$. A state over $\tau$ is a pair consisting of a frame and a heap. A heap $\mu$ is a partial mapping from an infinite and totally ordered set $\mathcal{L}$ of memory locations to objects; $\mu(\ell)$ is the object bound to $\ell \in \mathcal{L}$ in the heap $\mu$. An object $o \in O$ is a pair consisting of a class tag o.tag $\in \mathcal{K}$, and a frame o.frm which maps its fields into $\mathcal{V}=\mathbb{Z} \cup \mathcal{L} \cup\{$ null $\}$. For simplicity, it is assumed that no two fields $\kappa$.fld and $\kappa^{\prime}$.fld with the same field name can be declared in a program, so that fld will be usually a shorthand for $\kappa$.fld; this is not a significant restriction w.r.t. Java since the actual field to which a Java expression $v$.fld may refer to can be (and actually is) known statically. Shorthands are used: $o$.fld for $o$.frm(fld); $\mu[\ell \mapsto o]$ to modify the heap $\mu$ such that a location $\ell$ contains the object $o$; and $\mu[\ell$.fld $\mapsto$ val $]$ to modify the value of the field fld of the object $\mu(\ell)$ to $v a l \in \mathcal{V}$. A frame $\phi$ maps variables in $\operatorname{dom}(\tau)$ to $\mathcal{V}$. For $v \in \operatorname{dom}(\tau), \phi(v)$ refers to the value of $v$, and $\phi[v \mapsto v a l]$ is the frame where $v$ has been set to $v a l$, or defined to be val if $v \notin \operatorname{dom}(\phi)$. The set of states over $\tau$ is

$$
\Sigma_{\tau}=\left\{\begin{array}{l|l}
\langle\phi, \mu\rangle & \begin{array}{l}
\text { 1. } \phi \text { is a frame over } \tau, \mu \text { is a heap, and both are well-typed } \\
\text { 2. } \operatorname{rng}(\phi) \cap \mathcal{L} \subseteq \operatorname{dom}(\mu) \\
\text { 3. } \forall \ell \in \operatorname{dom}(\mu) . \operatorname{rng}(\mu(\ell) . \operatorname{frm}) \cap \mathcal{L} \subseteq \operatorname{dom}(\mu)
\end{array}
\end{array}\right\}
$$

\footnotetext{
${ }^{4}$ For simplicity, int fields will be often ignored since they have no impact on the heap.
} 


$$
\begin{aligned}
& E_{\tau}^{\iota} \llbracket n \rrbracket(\sigma)=\left\langle\sigma^{f}[\rho \mapsto n], \sigma^{h}\right\rangle \\
& E_{\tau}^{\iota} \llbracket \text { null } \rrbracket(\sigma)=\left\langle\sigma^{f}[\rho \mapsto n u l l], \sigma^{h}\right\rangle \\
& E_{\tau}^{\iota} \llbracket \text { new } \kappa \rrbracket(\sigma)=\left\langle\sigma^{f}[\rho \mapsto \ell], \sigma^{h}[\ell \mapsto n e w o b j(\kappa)]\right\rangle \text { where } \ell \notin \operatorname{dom}\left(\sigma^{h}\right) \\
& E_{\tau}^{\iota} \llbracket v \rrbracket(\sigma)=\left\langle\sigma^{f}\left[\rho \mapsto \sigma^{f}(v)\right], \sigma^{h}\right\rangle \\
& E_{\tau}^{\iota} \llbracket v \cdot f l d \rrbracket(\sigma)=\left\langle\sigma^{f}\left[\rho \mapsto \sigma^{h}\left(\sigma^{f}(v)\right) . f l d\right], \sigma^{h}\right\rangle \\
& E_{\tau}^{\iota} \llbracket \llbracket \exp _{1} \oplus \exp _{2} \rrbracket(\sigma)=\left\langle\sigma^{f}\left[\rho \mapsto \sigma_{1}^{f}(\rho) \oplus \sigma_{2}^{f}(\rho)\right], \sigma_{2}^{h}\right\rangle \text { where } \\
& \sigma_{1}=E_{\tau}^{\iota} \llbracket \exp _{1} \rrbracket(\sigma) \text { and } \sigma_{2}=E_{\tau}^{\iota} \llbracket \llbracket \exp _{2} \rrbracket\left(\left\langle\sigma^{f}, \sigma_{1}^{h}\right\rangle\right) \\
& E_{\tau}^{\iota} \llbracket v_{0} \cdot m t h\left(v_{1}, \ldots, v_{n}\right) \rrbracket(\sigma)=\left\langle\sigma^{f}\left[\rho \mapsto \sigma_{2}^{f}(\text { out })\right], \sigma_{2}^{h}\right\rangle \text { where } \sigma_{2}=\iota(\mathrm{mth})\left(\sigma_{1}\right) \text { s.t. } \sigma_{1} \text { is } \\
& \sigma_{1}^{h}=\sigma^{h} ; \sigma_{1}^{f}(\text { this })=\sigma^{f}\left(v_{0}\right) ; \forall 1 \leq i \leq n . \sigma_{1}^{f}\left(w_{i}\right)=\sigma^{f}\left(v_{i}\right) ; \\
& \text { and } \mathrm{mth}=\operatorname{lookup}\left(\sigma, v_{0} \cdot m \operatorname{th}\left(v_{1}, \ldots, v_{n}\right)\right) \text {; } \\
& C_{\tau}^{t} \llbracket \mathbf{s k i p} \rrbracket(\sigma)=\sigma \\
& C_{\tau}^{l} \llbracket v:=\exp \rrbracket(\sigma)=\left\langle\sigma^{f}\left[v \mapsto \sigma_{e}^{f}(\rho)\right], \sigma_{e}^{h}\right\rangle \\
& C_{\tau}^{l} \llbracket\left[v \cdot f l d:=\exp \rrbracket(\sigma)=\left\langle\sigma^{f}, \sigma^{h}\left[\ell . f l d \mapsto \sigma_{e}^{f}(\rho)\right]\right\rangle \text { where } \ell=\sigma^{f}(v)\right. \\
& C_{\tau}^{l} \llbracket \text { if } \exp \text { then } \operatorname{com}_{1} \rrbracket(\sigma)=\text { if } \sigma_{e}^{f}(\rho) \neq 0 \text { then } C_{\tau}^{\iota} \llbracket \operatorname{com}_{1} \rrbracket(\sigma) \text { else } C_{\tau}^{l} \llbracket \operatorname{com}_{2} \rrbracket(\sigma) \\
& C_{\tau}^{L} \llbracket \text { while } \exp \text { do } \operatorname{com} \rrbracket(\sigma)=\delta(\sigma) \text { where } \delta \text { is the least fixpoint of } \\
& \lambda w . \lambda \sigma \text {. if } \sigma_{e}^{f}(\rho) \neq 0 \text { then } w\left(C_{\tau}^{\iota} \llbracket \operatorname{com} \rrbracket(\sigma)\right) \text { else } \sigma \\
& C_{\tau}^{\iota} \llbracket \text { return } \exp \rrbracket(\sigma)=\left\langle\sigma^{f}\left[\text { out } \mapsto \sigma_{e}^{f}(\rho)\right], \sigma_{e}^{h}\right\rangle \\
& C_{\tau}^{l} \llbracket \operatorname{com}_{1} ; \operatorname{com}_{2} \rrbracket(\sigma)=C_{\tau}^{l} \llbracket \operatorname{com}_{2} \rrbracket\left(C_{\tau}^{\iota} \llbracket \operatorname{com}_{1} \rrbracket(\sigma)\right)
\end{aligned}
$$

Fig. 1. Denotations for expressions and commands. The state $\sigma_{e}$ is $E_{\tau}^{\iota} \llbracket \exp \rrbracket(\sigma)$.

Given $\sigma \in \Sigma_{\tau}, \sigma^{f}$ and $\sigma^{h}$ refer to its frame and its heap, respectively. The lattice $\mathcal{I}_{b}^{\tau}=$ $\left\langle\wp\left(\Sigma_{\tau}\right), T, \perp, \cap, \cup\right\rangle$ defines the concrete domain, where $T=\Sigma_{\tau}$ and $\perp=\emptyset$.

A denotation $\delta$ over type environments $\tau_{1}$ and $\tau_{2}$ is a partial map from $\Sigma_{\tau_{1}}$ to $\Sigma_{\tau_{2}}$ : it describes how the state changes when some code is executed. The set of denotations from $\tau_{1}$ to $\tau_{2}$ is $\Delta\left(\tau_{1}, \tau_{2}\right)$. An interpretation $\iota$ is a special denotation which gives a meaning to methods in terms of their input and output variables: it maps methods to denotations, such that $\iota(\mathrm{mth}) \in \Delta\left(\mathrm{mth}^{i},\{\right.$ out $\left.\}\right)$ for each $\mathrm{mth}$. The variable out is a special variable denoting the return value of methods. Let $\Gamma$ be the set of all interpretations.

Denotations for expressions and commands are depicted in Figure 1 An expression denotation $E_{\tau}^{\iota} \llbracket \exp \rrbracket$ maps states from $\Sigma_{\tau}$ to states from $\Sigma_{\tau \cup\{\rho\}}$, where $\rho$ is a special variable for storing the value of exp. A command denotation $C_{\tau}^{l} \llbracket c o m \rrbracket$ maps states to states, in presence of $\iota \in \Gamma$. The function newobj $(\kappa)$ creates a new instance of $\kappa$ with int fields initialized to 0 and reference fields initialized to $n u l l$, while newloc $\left(\sigma^{h}\right)$ returns the first free location, i.e., the first $\ell \notin \operatorname{dom}\left(\sigma^{h}\right)$ according to the total ordering on locations. The function lookup resolves the method call according to the runtime type of the object, and returns the signature of the method to be invoked. The concrete denotational semantics of a program is defined as the least fixpoint (lfp) of the following transformer of interpretations [Bossi et al. 1994].

\section{Definition 2.1}

The denotational semantics of a program $P$ is the lfp of

$$
\begin{aligned}
T_{P}(\iota) & =\left\{\mathrm{mth} \mapsto \lambda \sigma \in \Sigma_{\mathrm{mth}^{i}} \cdot \exists \tau \backslash \text { out. } C_{\mathrm{mth}^{s} \cup\{\text { out }\}} \|\left[\mathrm{mth}^{b} \rrbracket(\operatorname{ext}(\sigma, \mathrm{mth}))\right\}_{\mathrm{mth} \in P}\right. \\
\text { where } \operatorname{ext}(\sigma, \mathrm{mth}) & =\left\langle\sigma^{f}\left[\forall v \in \mathrm{mth}^{l} \cup\{\text { out }\} . v \mapsto 0 / \text { mull }\right], \sigma^{h}\right\rangle .
\end{aligned}
$$

The denotation for a method signature mth $\in P$ is computed by $T_{P}$ as follows: it (1) extends (using $\operatorname{ext}(\sigma, \mathrm{mth}))$ the input state $\sigma \in \Sigma_{\mathrm{mth}^{i}}$ such that local variables are set to 0 or null; 
(2) computes the denotation of the code of mth, using $C_{m_{t h}{ }^{s} \cup\{o u t\}}^{\iota} \llbracket-\rrbracket$; and (3) restricts the resulting denotation to out, using $\exists \tau \backslash$ out.

\section{THE ABSTRACT DOMAINS}

This section formalizes the analysis $\boldsymbol{y}_{\text {fld }}$ by means of Abstract Interpretation [Cousot and Cousot 1977], relying on the notion of abstract domain. The following example shows a class hierarchy which will be used in the rest of this section.

Example 3.1 ((class hierarchy)). Let the class hierarchy $\langle\mathcal{K},<\rangle$ under study be defined as follows. Objects of class Emp model employees, which can be of level 1 (L1) or 2 (L2). An employee has one main device (mD), which is a laptop (LP); level-2 employees also have a tablet (TB) as an accessory device (aD) which is associated (Ink) to a laptop. Devices (Dev) are also associated to their owner (owner).
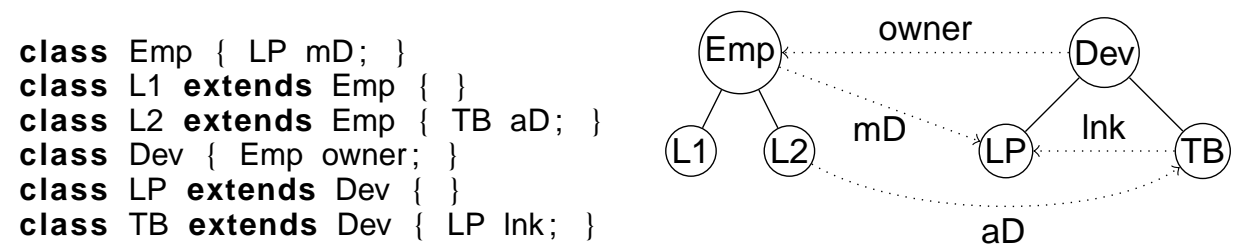

In the figure, solid lines correspond to <; dotted lines represent fields.

\subsection{Background in Logic}

A Boolean function is a function $f:$ Bool $^{n} \mapsto$ Bool with $n \geq 0$, and can be represented as a propositional formula over a set $X$ with cardinality $n$. In this paper, Boolean functions and propositional formulæ will be used interchangeably. Moreover, a truth assignment of Boolean variables will be often represented as the set of variables which are true under that assignment. In this framework, $X$ will be the set $\mathcal{P}=\left\{\right.$ flet $^{\prime} \mid$ fld $\left.\in \mathcal{F}\right\}$ of propositions flet corresponding to program fields. Such propositions are called $f$-propositions. Propositional formulæ over $\mathcal{P}$ are called path-formulæ. As usual, a truth assignment $\omega \subseteq \mathcal{P}$ is a model of a path-formula $F$ if $F$ evaluates to true under $\omega$. The set of models of $F$ is denoted by $\operatorname{modelsp}(F)$.

The path-formula $\langle\omega\rangle$ is defined as $\wedge\left\{\right.$ flet $^{\lambda} \mid$ flet $\left.\in \omega\right\} \wedge \wedge\{\neg$ flet $\mid$ fled $\in \mathcal{P} \backslash \omega\}$, and represents the formula whose only model is $\omega$. An important special case is $\langle\emptyset\rangle=\wedge\{\neg$ flet $\mid$ flet $\in \mathcal{P}\}$; moreover, $\left\langle\right.$ fld $\left.\right|_{1}, .$. fld $\left.d_{k}\right\rangle$ will be a shorthand for $\left\langle\left\{f l d_{1} . . . f l d d_{k}\right\rangle\right\rangle$.

A formula $F$ is monotone if, for every two assignments $\omega$ and $\omega^{\prime}, \omega \subseteq \omega^{\prime}$ and $\omega \in$ models $\mathcal{P}(F)$ imply $\omega^{\prime} \in$ models $_{\mathcal{P}}(F)$. It is positive if $\mathcal{P} \in$ models $_{\mathcal{P}}(F)$. It is definite if, for every two assignments $\omega^{\prime}$ and $\omega^{\prime \prime}, \omega^{\prime} \in$ modelsp $(F)$ and $\omega^{\prime \prime} \in \operatorname{model} s p(F)$ implies $\omega^{\prime} \cap \omega^{\prime \prime} \in \operatorname{model} s p(F)$. Finally, set conjunction $\Lambda X$ and set disjunction $\vee X$ will be, respectively, true and false whenever $X=\emptyset$. $\mathcal{P F}$ denotes the set of all path-formulæ.

\subsection{Paths, cycles, and fields}

The abstract domains used by $\boldsymbol{Y}_{\text {fld }}$ are based on the notion of reachable heap locations, i.e., the part of the heap which can be reached starting from a given location (or the variable pointing to it). Given a heap $\mu$, a path $\pi$ from $\ell^{\prime} \in \operatorname{dom}(\mu)$ to $\ell^{\prime \prime} \in \operatorname{dom}(\mu)$ is a sequence $\left\langle\ell_{0}, . ., \ell_{k}\right\rangle$ of locations such that (1) $k \geq 0$; (2) $\ell_{0}=\ell^{\prime}$; (3) $\ell_{k}=\ell^{\prime \prime}$; and (4) for every $0 \leq i \leq k-1$, it holds that $\ell_{i+1} \in \operatorname{rng}\left(\mu\left(\ell_{i}\right)\right.$.frm $)$, i.e., $\ell_{i+1}$ is the location bounded to a field of the object to which $\ell_{i}$ is bound. The length of a path $\left\langle\ell_{0}, . ., \ell_{k}\right\rangle$ is $k$; empty paths are those with length 0 . A cycle is a path from $\ell$ to $\ell$ itself; it is an empty cycle if its length is 0 . Given $\pi_{1}=\left\langle\ell_{0}, . ., \ell_{k}\right\rangle$ and $\pi_{2}=\left\langle\ell_{k}, . ., \ell_{m}\right\rangle$, the concatenation $\pi_{1} \cdot \pi_{2}$ is the path $\left\langle\ell_{1}, . ., \ell_{k}, . ., \ell_{m}\right\rangle$. 
Definition 3.2 ((reachable locations, similar to [Rossignoli and Spoto 2006])). The set of all reachable locations from $\ell \in \operatorname{dom}(\mu)$ is $R(\mu, \ell)=\cup\left\{R^{i}(\mu, \ell) \mid i \geq 0\right\}$, where $R^{0}(\mu, \ell)=\{\ell\}$, and $R^{i+1}(\mu, \ell)$ is $\cup\left\{\operatorname{rng}\left(\mu\left(\ell^{\prime}\right)\right.\right.$.frm $\left.) \cap \mathcal{L} \mid \ell^{\prime} \in R^{i}(\mu, \ell)\right\}$.

The rest of this section is developed in the context of a type environment $\tau$, which will be often left implicit. $\boldsymbol{Y}_{\text {fld }}$ considers fields or field identifiers when collecting information about paths; to this end, domains introduced in Sections 3.3 and 3.4 are based on the notion of field-reachable heap locations, i.e., the part of the heap which can be reached from a location by traversing (dereferencing) certain fields.

Definition 3.3 ((field traversal)). A path $\pi$ is said to traverse a field $f l d \in \mathcal{F}$ in the state $\sigma$ if (1) it is a path in $\sigma^{h} ;(2) \pi=\left\langle\ell_{0}, . ., \ell_{i}, . ., \ell_{i+1}, . ., \ell_{k}\right\rangle$ with $k>i \geq 0$; (3) an object $o$ of class $\kappa^{\prime} \leq \kappa$ (i.e., o.tag $=\mathcal{K}^{\prime}$ ) is stored in $\ell_{i}$ (i.e., $\sigma^{h}\left(\ell_{i}\right)=o$ ); and (4) o.fld points to the location $\ell_{i+1}$, i.e., o.frm $($ fld $)=\ell_{i+1}$.

Example 3.4 ((field traversal)). The path depicted below is compatible with the class hierarchy of Example 3.1, and traverses fields L2.aD, TB.Ink and Dev.owner.

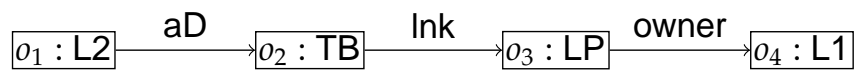

Definition 3.5 ((p-satisfaction)). A path $\pi$ is said to $p$-satisfy an f-proposition flet iff it traverses fld. Given a path-formula $F$, the p-satisfaction of $F$ by $\pi$, written $\pi \rightarrow F$, follows the usual logical rules:

$$
\begin{aligned}
\pi \rightarrow \text { flet iff } \pi \text { traverses fld } & \pi \rightarrow \neg F \text { iff } \pi \rightarrow F \text { does not hold } \\
\pi \rightarrow F^{\prime} \wedge F^{\prime \prime} \text { iff } \pi \rightarrow F^{\prime} \text { and } \pi \rightarrow F^{\prime \prime} & \pi \rightarrow F^{\prime} \vee F^{\prime \prime} \text { iff } \pi \rightarrow F^{\prime} \text { or } \pi \rightarrow F^{\prime \prime}
\end{aligned}
$$

As usual, true stands for a tautology, and false stands for a contradiction. Ordering on pathformulæ is logical implication: $F^{\prime} \leq F^{\prime \prime}$ iff $F^{\prime} \Rightarrow F^{\prime \prime}$ is valid. The meaning is straightforward: for every $\pi$, if $F^{\prime} \leq F^{\prime \prime}$ and $\pi \rightarrow F^{\prime}$, then $\pi \rightarrow F^{\prime \prime}$.

Example 3.6 ((p-satisfaction)). The path of Example 3.4 p-satisfies $\mathrm{aD} \wedge$ owner , and any path-formula which is implied by it, such as $\mathrm{aE}$. On the other hand, it does not p-satisfy ᄀowner $\vee \mathrm{mO}$.

A truth assignment $\omega \subseteq \mathcal{P}$ is said to be viable if there exists some path $\pi$ in some state $\sigma$ which p-satisfies $\langle\omega\rangle$. To rule out non-viable truth assignments allows obtaining a Galois insertion (i.e., without superfluous elements in the abstract domain) rather than a Galois connection in the definition of the abstract domains for reachability and cyclicity.

Lemma 3.7 ((viability)). The viability of a truth assignment $\omega$ is decidables.

Note that viability is not related to an assignment that satisfies a formula, but rather to a property of the assignment itself: that it can represent a "real" path in a "real" heap according to class declarations.

Example 3.8 ((viability of truth assignments)). Given the class hierarchy introduced in Example 3.1 the truth assignment $\{\mathrm{aD}$, Ink, owner\} is viable, as shown by the path of Example 3.4 On the other hand, $\{\mathrm{mD}, \mathrm{Ink}\}$ is not viable. In fact, a path only traversing $\mathrm{mD}$ and Ink should contain at least one TB object $o_{\mathrm{TB}}$ and one LP object $o_{\mathrm{LP}}$ since Ink must be traversed. It must also include one Emp (or a subclass) object $o_{\mathrm{Emp}}$ since $\mathrm{mD}$ must also be traversed. Suppose $o_{\mathrm{TB}}$ is the first object on the path: then the second must be $o_{\mathrm{LP}}$ since owner cannot be traversed, and there is no way to reach $o_{\mathrm{Emp}}$. On the other hand, if the $o_{\mathrm{LP}}$ is the first object, then no other object can be reached without traversing owner. Finally, if $o_{\text {Emp }}$ is the

${ }^{5}$ Proofs are available in Appendix 
first object, then the second one must be $o_{\mathrm{LP}}$, and, again, no other object can be reached from it.

Definition 3.9 ((equivalence)). The set $\mathcal{P F}$ of path-formulæ can be partitioned according to the following equivalence relation: $F$ and $G$ are equivalent unless there is a path in some state which p-satisfies one and only one of them. Note that this relation is "coarser" than (i.e., implied by) standard logical equivalence since the discriminating path must be compatible with the class hierarchy.

Lemma 3.10 ((EQuivalence)). The equivalence of two path-formulæ is decidable.

In the following, $\mathcal{P F} \equiv$ will be $\mathcal{P F}$ with the equivalence relation of Definition 3.9

\subsection{The Field-Reachability domain}

First, the definition of field-reachability between program variables is given.

Definition 3.11 ((field-reachability on variables)). A variable $v$ is said to reach another variable $w$ in $\sigma$ if there exists a path from $\sigma^{f}(v)$ to $\sigma^{f}(w)$. Moreover, given some $F, v$ is said to F-reach $w$ in $\sigma$ if every path from $\sigma^{f}(v)$ to $\sigma^{f}(w)$ p-satisfies $F$. This definition implies that any variable $v$ false-reaches $w$ if and only if there is no path between them.

Example 3.12 ((reachability)). Consider the heap depicted below, based on Example 3.1

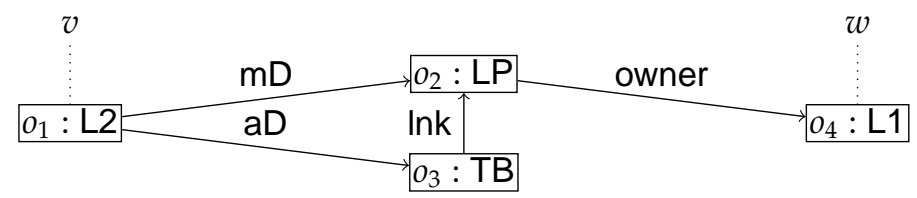

Among the path-formulæ $F$ such that $v F$-reaches $w$, there are:

$\begin{array}{ll}\mathrm{mD} \vee \mathrm{aD} & : \text { all paths traverse either } \mathrm{mD} \text { or } \mathrm{aD} \\ \text { Owher } & : \text { all paths traverse owner } \\ \neg \mathrm{mk} \vee \neg \mathrm{mD} & : \text { at most one between Ink and } \mathrm{mD} \text { is traversed }\end{array}$

An extension of the equivalence relation on path-formulæ is needed here: $\mathcal{P F}_{\equiv}^{2}$ is a function which takes a pair of variables $(v, w)$, and returns the set $\mathcal{P F}$ equipped by the following equivalence relation $\equiv^{v, w}: F \equiv^{v, w} G$ unless there is a path from $\sigma^{f}(v)$ to $\sigma^{f}(w)$ in some state which p-satisfies one and only one between $F$ and $G$. The only difference w.r.t. the original $\equiv$ is that the path must connect $v$ to $w$.

The reachability abstract domain is formalized similarly to $\mathcal{I}_{r}^{\tau}$ [Genaim and Zanardini 2013], and is actually a refinement of it (Section 3.6). The next definition shows the lattice of abstract values representing reachability between variables. In the following, functions are often represented by $\lambda$-notation, and $\tau$ is omitted.

Definition 3.13. The field-reachability abstract domain is the complete lattice

$$
\overline{\mathcal{I}}_{r}=\left\langle\overline{\mathcal{R}}, \overline{\underline{\Xi}}_{r}, \bar{\beth}_{r}, \overline{\mathrm{T}}_{r}, \bar{\Gamma}_{r}, \bar{\sqcup}_{r}\right\rangle
$$

- the set $\overline{\mathcal{R}}$ is the set of functions whose domain is $\mathcal{X} \times \mathcal{X}$, and that return an element of $\mathcal{P F} \mathcal{F}_{\equiv}^{2}(v, w)=\mathcal{P F} \mathcal{F}_{\equiv, v, v}$ for a pair of arguments $(v, w)$;

- $\overline{\underline{E}}_{r}$ is $\leq$ on path-formulæ, applied point-wise;

$-\bar{I}_{r}=\lambda(v, w)$. false and $\overline{\mathrm{T}}_{r}=\lambda(v, w)$.true;

$-f^{\prime} \bar{\Pi}_{r} f^{\prime \prime}=\lambda(v, w) \cdot f^{\prime}(v, w) \wedge f^{\prime \prime}(v, w)$ and $f^{\prime} \square_{r} f^{\prime \prime}=\lambda(v, w) \cdot f^{\prime}(v, w) \vee f^{\prime \prime}(v, w)$. 
The meaning of an abstract value $\bar{I}_{r}$ is the following: it represents all the states where, for every $v$ and $w$ (possibly the same variable), all paths from $v$ to $w$ p-satisfy $F=\bar{I}_{r}(v, w)$. Note that $F \not \equiv$ false does not mean that there is some path from $v$ to $w$ in a concrete state: this a "possible" analysis, so that non-reachability is always a possibility. On the other hand, $\bar{I}_{r}(v, w)=$ false excludes reachability since no path p-satisfies false. The bottom $\bar{I}_{r}$ models the (non-empty) set of all states where all reference variables are null, whereas $\overline{\mathrm{T}}_{r}$ represents $\Sigma_{\tau}$. Note that $\bar{I}_{r}(v, v) \geq\langle\emptyset\rangle$ (recall that $\langle\emptyset\rangle$ is $\bigwedge\{\neg$ flet $\mid$ flet $\in \mathcal{P}\}$ ) whenever $v$ is not null. Unlike $\mathcal{I}_{r}^{\tau}$, this abstract domain can also represent aliasing [Hind 2001] because empty paths are also considered. This explains the different definition of the bottom element in $\overline{\mathcal{I}}_{r}$ and $\mathcal{I}_{r}^{\tau}$.

Example 3.14 ((abstract values)). In a program where $\kappa . f l d_{1}$ and $\kappa . f l d_{2}$ are the only fields, the abstract value $\bar{I}_{r}$ such that

$$
\begin{aligned}
\bar{I}_{r}(v, v) & =\left(\neg f l d_{1} \wedge \neg f l d_{2}\right) \vee\left(\text { fld }_{1} \wedge \text { fld } d_{2}\right) \\
\bar{I}_{r}(v, w)=\bar{I}_{r}\left(v, w^{\prime}\right) & =f \text { fld }_{1} \\
\bar{I}_{r}(w, v)=\bar{I}_{r}\left(w^{\prime}, v\right) & =\text { false } \\
\bar{I}_{r}(w, w)=\bar{I}_{r}\left(w, w^{\prime}\right)=\bar{I}_{r}\left(w^{\prime}, w\right)=\bar{I}_{r}\left(w^{\prime}, w^{\prime}\right) & =\neg \text { fld }_{1} \wedge \neg f l d_{2}
\end{aligned}
$$

represents heaps (a) and (b), but not (c). The last line allows $w$ and $w^{\prime}$ to alias, and this is compatible with all heaps: in the first, $w^{\prime}$ is null, so that there are no paths starting from it, not even empty paths; in the second, they actually alias; in the third, self-aliasing holds for both, but they do not alias with each other. Heap (a) is represented by $\bar{I}_{r}(v, v)$ since $v$ is not cyclic (only self-aliasing), by $\bar{I}_{r}(v, w)$ because the path from $v$ to $w$ actually traverses $f l d_{1}$, and by $\bar{I}_{r}\left(v, w^{\prime}\right)$ since there are no paths between $v$ and $w^{\prime}$. Note that $\bar{I}_{r}(v, w)$ also allows paths to traverse $f l d_{2}$, as in this case. Heap (b) is also represented by $\bar{I}_{r}(v, v)$ because $v$ is self-reachable by a path traversing both fields, and there is no other path only traversing one of them. It is also represented by $\bar{I}_{r}(v, w)$ and $\bar{I}_{r}\left(v, w^{\prime}\right)$ since $v$ does not need to actually reach $w$ or $w^{\prime}$. Heap (c) is not represented by $\bar{I}_{r}(w, v)$ because false means that there can be no reachability from $w$ to $v$.

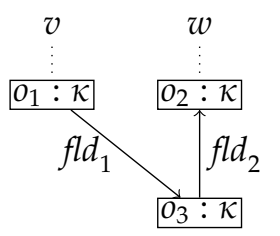

(a)

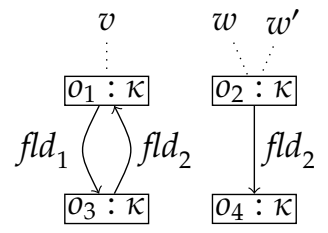

(b)

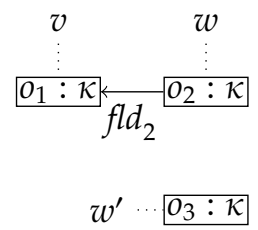

(c)

In general, the representation of most path-formulæ can be simplified by using the \langle\rangle notation: for example, $\bar{I}_{r}(v, v)$ can be written as $\langle\emptyset\rangle \vee\left\langle\right.$ fld $\left._{1}, f l d_{2}\right\rangle$.

Definition 3.15 ((abstraction and concretization)). The abstraction and concretization functions between $\overline{\mathcal{I}}_{r}$ and the concrete domain $\mathcal{I}_{b}^{\tau}$ are:

$$
\begin{aligned}
& \bar{\alpha}_{r}(\sigma)=\lambda(v, w) . \wedge\{F \mid v F \text {-reaches } w \text { in } \sigma\} \\
& \bar{\alpha}_{r}\left(I_{\mathrm{b}}\right)=\lambda(v, w) . \bigvee\left\{\bar{\alpha}_{r}(\sigma)(v, w) \mid \sigma \in I_{\mathrm{b}}\right\} \\
& \bar{\gamma}_{r}\left(\bar{I}_{r}\right)=\left\{\sigma \in \Sigma_{\tau} \mid \forall v, w \in \tau . \exists F \leq \bar{I}_{r}(v, w) . v F \text {-reaches } w \text { in } \sigma\right\}
\end{aligned}
$$

$\bar{\alpha}_{r}$ is computed as follows: for every $\sigma$, the conjunction of all the $F$ s.t. $v F$-reaches $w$ comes to be the strongest condition p-satisfied by all paths from $v$ to $w$ in $\sigma$ (recall that $F$-reachability means that all paths p-satisfy $F$ ). All strongest conditions are combined by disjunction on states. On the other hand, $\bar{\gamma}_{r}$ is the adjoint function required by Lemma 3.16. As expected, given $\bar{I}_{r}$ s.t. $\bar{I}_{r}(v, w)=G$, a state where $v$ does not reach $w$ is still compatible with $\bar{\gamma}_{r}\left(\bar{I}_{r}\right)$ (i.e., 
it belongs to the concretization unless other variables make it incompatible) since false $\leq G$, and $v$ false-reaches $w$.

Lemma 3.16 ((INSERTION)). $\bar{\alpha}_{r}$ and $\bar{\gamma}_{r}$ define a Galois insertion between $\overline{\mathcal{I}}_{r}$ and $\mathcal{I}_{b}^{\tau}$.

\subsection{The Field-Cyclicity domain}

The abstract domain $\bar{I}_{c}$ for cyclicity is similar to $\bar{I}_{r}$, so that most technical details will not be repeated. The following definition is similar to Definition 3.11.

Definition 3.17 ((field-cyclic variables)). A variable $v$ is said to be cyclic in a state $\sigma$ if there exists a path from $\sigma^{f}(v)$ containing a cycle. Given a path-formula $F, v$ is said to be F-cyclic in $\sigma$ if all cycles reachable from $\sigma^{f}(v)$ in $\sigma$ p-satisfy $F$.

Note that the p-satisfaction of $F$ is not required for the whole path starting at $\sigma^{f}(v)$; rather, it must hold when only the cyclic part of the path is considered.

A new extension of the equivalence relation on path-formulæ is needed in order to deal with cyclicity: $\mathcal{P F} \equiv$ will be a function which takes a variable $v$, and returns the set $\mathcal{P F}$ equipped by the equivalence relation $\equiv^{v}: F \equiv^{v} G$ unless there is a path in some state which (1) starts at $\sigma^{f}(v)$; and (2) contains a cycle which p-satisfies one and only one between $F$ and $G$.

Definition 3.18 ((cyclicity abstract domain)). The abstract domain for field-cyclicity is similar to the field-reachability domain: it is the complete lattice

$$
\overline{\mathcal{I}}_{c}=\left\langle\bar{y}, \bar{\Xi}_{c}, \bar{I}_{c}, \overline{\mathrm{T}}_{c}, \bar{\Pi}_{c}, \bar{\sqcup}_{c}\right\rangle
$$

where $\overline{\mathcal{Y}}$ is the set of functions mapping each reference variable $v$ to an element of $\mathcal{P \mathcal { F }}{ }_{\equiv}^{1}(v)$; $\underline{\Xi}_{c}$ is $\leq$, applied point-wise; $\bar{I}_{c}=\lambda v$.false, and $\bar{\top}_{c}=\lambda v$.true; and $\bar{\Pi}_{c}$ and $\bar{\Xi}_{c}$ are, respectively, $\wedge$ and $\vee$, applied point-wise.

Definition 3.19 ((abstraction and concretization)). The functions

$$
\begin{aligned}
& \bar{\alpha}_{c}(\sigma)=\lambda v \cdot \bigwedge\{F \mid v \text { is F-cyclic in } \sigma\} \\
& \bar{\alpha}_{c}\left(I_{b}\right)=\lambda v \cdot \bigvee\left\{\bar{\alpha}_{c}(\sigma)(v) \mid \sigma \in I_{b}\right\} \\
& \bar{\gamma}_{c}\left(\bar{I}_{c}\right)=\left\{\sigma \in \Sigma_{\tau} \mid \forall v \in \tau . \exists F \leq \bar{I}_{c}(v) . v \text { is F-cyclic in } \sigma\right\}
\end{aligned}
$$

are the abstraction and concretization functions between $\bar{I}_{c}$ and $\mathcal{I}_{b}^{\tau}$.

Lemma 3.20 ((InSERTION)). $\quad \bar{\alpha}_{c}$ and $\bar{\gamma}_{c}$ define $a$ Galois insertion between $\overline{\mathcal{I}}_{c}$ and $\mathcal{I}_{b}^{\tau}$.

An abstract value such that $\bar{I}_{c}(v)=F$ represents states where all cyclic sub-paths of paths starting at $\sigma^{f}(v)$, if any, have to p-satisfy $F$. Similarly to reachability, the non-nullity of $v$ implies that $\bar{I}_{c}(v) \geq\langle\emptyset\rangle$ since there always exists an empty path from $\sigma^{f}(v)$ to $\sigma^{f}(v)$.

Example 3.21. Let $\bar{I}_{c}$ be an abstract state, and $\bar{I}_{c}(v)=\langle\emptyset\rangle \vee\left\langle\right.$ fld $_{1}$, fld $\left.\bar{d}_{2}\right\rangle$ be the path-formula whose only models are $\emptyset$ and $\left\{\right.$ fld $_{1}$, fld $\left._{2}\right\}$. Consider the three heaps below.

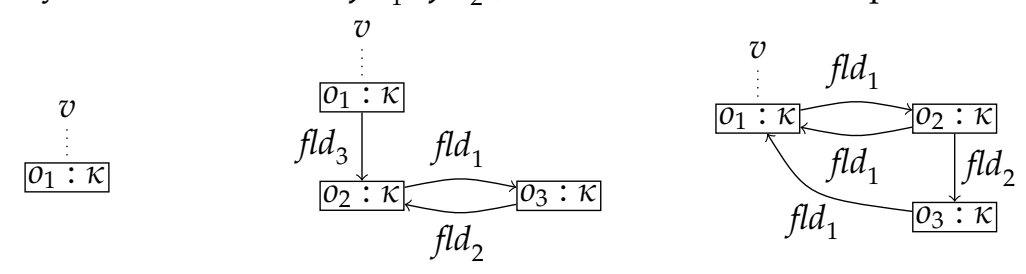


The heap depicted on the left is correctly represented by this abstract value because the empty cycle from $\sigma^{f}(v)$ to $\sigma^{f}(v)$ p-satisfies $\emptyset$ which is a model of $\bar{I}_{c}(v)$. The second heap is also represented because the only non-trivial cycle starts from $o_{2}$ and traverses both fld $_{1}$ and $f l d_{2}$; note that $\bar{I}_{c}(v)$ does not need to account for $f l d_{3}$ since this field is not traversed by the cycle. On the other hand, the heap on the right is not correctly represented because there is a cycle only traversing $\mathrm{fld}_{1}$, and $\left\{\mathrm{fll}_{1}\right\}$ is not a model of $\bar{I}_{c}(v)$.

\subsection{The reduced product}

The (direct) product of the abstract domains presented in this section is the set of pairs $\bar{I}_{r c}=\left(\bar{I}_{r}, \bar{I}_{c}\right)$, and the theory of Abstract Interpretation guarantees that it identifies a Galois connection with $\gamma\left(\bar{I}_{r}, \bar{I}_{c}\right)=\bar{\gamma}_{r}\left(\bar{I}_{r}\right) \cap \bar{\gamma}_{c}\left(\bar{I}_{c}\right)$. In the following, $\bar{I}_{r c}\left(v_{1}, v_{2}\right)$ will be a shorthand for $\bar{I}_{r}\left(v_{1}, v_{2}\right)$, where $\bar{I}_{r}$ is the reachability part of $\bar{I}_{r c}$, and $\bar{I}_{r c}(v)$ will stand for $\bar{I}_{c}(v)$, where $\bar{I}_{c}$ is the cyclicity part of $\bar{I}_{r c}$.

Usually, the reduced product [Cousot and Cousot 1979] is more interesting than the direct product since it happens to generate a Galois insertion. It is obtained by "unifying" (by means of an equivalence relation) abstract values with the same concretization (i.e., representing the same set of concrete states). Two different abstract values are mapped to the same set of states when discrepancies between them do not "include" or "exclude" any state. This happens when $\bar{I}_{r}$ contains information which is not compatible with $\bar{I}_{c}$, similarly to the abstract domains used in the reachability-based analysis $\boldsymbol{y}_{r c}$ described in Section 1 [Genaim and Zanardini 2013, Lemma 4.7].

Example 3.22 ((reachability vs. cyclicity)). Let $\bar{I}_{r c}^{\prime}=\left(\bar{I}_{r}^{\prime}, \bar{I}_{c}\right)$ and $\bar{I}_{r c}^{\prime \prime}=\left(\bar{I}_{r}^{\prime \prime}, \bar{I}_{c}\right)$ only differ in the self-reachability part about $\mathrm{x}$; i.e., the cyclicity part is the same, and $\bar{I}_{c}(\mathrm{x})=\bar{I}_{r}^{\prime}(\mathrm{x}, \mathrm{x})=F$, but $\bar{I}_{r}^{\prime \prime}(\mathrm{x}, \mathrm{x})=G>F$. In this case, there is, in general, a set $X$ of states which are represented by $\bar{I}_{r}^{\prime \prime}$ but not by $\bar{I}_{r}^{\prime}$. In such states, this happens because of paths from $\mathrm{x}$ to $\mathrm{x}$ which p-satisfy $G$ but not $F$. However, states in $X$ are incompatible with $\bar{I}_{c}$ since a path from $\mathrm{x}$ to $\mathrm{x}$ is a cycle, but $\bar{I}_{c}(\mathrm{x})=F$ would not be $\mathrm{p}$-satisfied by such a path. Therefore, the difference $X$ between $\bar{\gamma}_{r}\left(\bar{I}_{r}^{\prime}(\mathrm{x}, \mathrm{x})\right)$ and $\bar{\gamma}_{r}\left(\bar{I}_{r}^{\prime \prime}(\mathrm{x}, \mathrm{x})\right)$ is a set of concrete states which are not represented by $\bar{\gamma}_{c}\left(\bar{I}_{c}(\mathbf{x})\right)$, so that $\bar{\gamma}_{r}\left(\bar{I}_{r}^{\prime}\right) \cap \bar{\gamma}_{c}\left(\bar{I}_{c}\right)=\bar{\gamma}_{r}\left(\bar{I}_{r}^{\prime \prime}\right) \cap \bar{\gamma}_{c}\left(\bar{I}_{c}\right)$. As a conclusion, both $\bar{I}_{r c}^{\prime}$ and $\bar{I}_{r c}^{\prime \prime}$ actually represent the same states.

Definition 3.23 ((normal form)). An abstract value $\left(\bar{I}_{r}, \bar{I}_{c}\right)$ is in normal form if, for every $v \in \tau, \bar{I}_{c}(v) \geq \bar{I}_{r}(v, v)$. The normalization $\mathcal{N}\left(\bar{I}_{r}, \bar{I}_{c}\right)$ is defined as

$$
\left(\mathcal{N}\left(\bar{I}_{r}, \bar{I}_{c}\right)\right)(v)=\bar{I}_{c}(v) \vee \bar{I}_{r}(v, v) \quad\left(\mathcal{N}\left(\bar{I}_{r}, \bar{I}_{c}\right)\right)(v, w)=\bar{I}_{r}(v, w)
$$

The reduced product of the reachability and cyclicity domains is the set of normal-form pairs $\left(\bar{I}_{r}, \bar{I}_{c}\right)$, as proved in the following lemma.

LemMa 3.24 ((REDUCED PRODUCT)). The lattice based on $\left\{\left(\bar{I}_{r}, \bar{I}_{c}\right) \mid \bar{I}_{r} \in \overline{\mathcal{R}}_{,} \bar{I}_{c} \in \overline{\mathcal{Y}}_{,},\left(\bar{I}_{r}, \bar{I}_{c}\right)\right.$ is in normal form $\}$

with $\bar{\gamma}_{r c}\left(\bar{I}_{r}, \bar{I}_{c}\right)=\bar{\gamma}_{r}\left(\bar{I}_{r}\right) \cap \bar{\gamma}_{c}\left(\bar{I}_{c}\right)$ is the reduced product between $\bar{I}_{r}$ and $\bar{I}_{c}$.

In the following, operators on abstract values will be extended to the reduced product. For example, since their domains are disjoint, $\bar{I}_{c} \sqcup \bar{I}_{r}$ will be the function $f$ such that (1) $f(v)=\bar{I}_{c}(v)$; and $(2) f(v, w)=\bar{I}_{r}(v, w)$. Moreover, reachability and cyclicity abstract values can be mixed: e.g., $\bar{I}_{r c} \overline{\bar{I}} \bar{I}_{r}$ will be the function $g$ such that $(1) g(v)=\bar{I}_{r c}(v)$ (i.e., the cyclicity component); and $(2) g(v, w)=\bar{I}_{r c}(v, w) \vee \bar{I}_{r}(v, w)$.

\subsection{Comparison with other approaches to the problem}

This section refers to reachability; its extension to cyclicity is straightforward. The domain $\overline{\mathcal{I}}_{r}$ presented in Section 3.3 is very expressive since it can predicate a number of interesting 
facts about paths. This section compares $\overline{\mathcal{I}}_{r c}$ with a number of abstract domains which are meant to tackle the same problem.

3.6.1. An abstract domain without field information. Such an abstract domain was inspired by a static analysis for C programs [Ghiya and Hendren 1996], and formalized as an abstract domain by [Genaim and Zanardini 2013]. It is structurally similar to $\overline{\mathcal{I}}_{r}$, but field information is not considered.

Definition 3.25 ((without fields Genaim and Zanardini 2013])). This abstract domain is the complete lattice $\mathcal{I}_{r}^{\tau}=\left\langle\wp\left(X^{\rightsquigarrow}\right), \subseteq, \emptyset, X, \cap, \cup\right\rangle$, where

$$
X^{\rightsquigarrow}=\left\{\begin{array}{l|l}
v \rightsquigarrow w & \begin{array}{l}
v, w \in \operatorname{dom}(\tau), \text { and there exist } \kappa_{1} \leq \tau(v) \text { and } \kappa_{2} \leq \tau(w) \\
\text { such that } \kappa_{2} \text { is reachable from } \kappa_{1}
\end{array}
\end{array}\right\}
$$

where the notion of reachability between classes is taken from |Secci and Spoto 2005|: " $\kappa_{2}$ is reachable from $\kappa_{1}$ " means that it is possible to have a heap where an object of class $\kappa_{1}$ reaches an object of class $\kappa_{2}$.

An abstract value $I_{r}$ is a set of statements _ $\rightsquigarrow_{-}$: if $v \rightsquigarrow w \notin I_{r}$, then the concretization of $I_{r}$ will not include any state where $v$ reaches $w . \mathcal{I}_{r}^{\tau}$ is an abstraction of $\overline{\mathcal{I}}_{r}$.

Lemma 3.26. The abstract domain $\mathcal{I}_{r}^{\tau}$ is an abstraction of $\overline{\mathcal{I}}_{r}$.

As already mentioned, $\overline{\mathcal{I}}_{r}$ is also able to represent aliasing [Hind 2001]. Indeed, it is also a refinement of the standard abstract domain for aliasing analysis.

Lemma 3.27. The abstract domain $\overline{\mathcal{I}}_{r}$ is a refinement of the aliasing domain.

A sound abstract semantics based on $\mathcal{I}_{r}^{\tau}$ has been proposed by Genaim and Zanardini 2013]; very similar analyses can be found in the works by [Ghiya and Hendren 1996], and by [Nikolic and Spoto 2014]. In terms of precision, $\overline{\mathcal{I}}_{r}$ is more precise than $I_{r}^{i}$ since the field information can rule out states where paths do not p-satisfy a given formula. As discussed in the introduction, this is more evident when dealing with cyclicity, since such an extra information about cycles can lead to prove the termination of algorithms which traverse cyclic data structures. In terms of efficiency, it is clear that an abstract semantics based on $\overline{\mathcal{I}}_{r}$ and $\overline{\mathcal{I}}_{c}$ instead of $\mathcal{I}_{r}^{\tau}$ and $\mathcal{I}_{c}^{\tau}$ is more expensive since (1) operators on path-formulæ are more complex (e.g., $\odot$ in Section 4 ); and (2) the convergence of the global fixpoint (Section 4.4) can be slower; in fact, for every pair $(v, w)$, $\bar{I}_{r}$ allows ascending chains of path-formulæ whose length is exponential on the number of fields, while $\mathcal{I}_{r}^{\tau}$ only allows 2-long chains ("does not reach" < "reaches").

3.6.2. An abstract domain based solely on class reachability. Another abstract domain which can be studied is the one where just the class hierarchy is considered: a variable $v$ is regarded as potentially reaching $w$ whenever the class of $w$ is reachable from the class of $v$ [Secci and Spoto 2005]. Such a domain will be denoted by ${ }^{k} \mathcal{I}_{r}$; needless to say, it is an abstraction of both $\mathcal{I}_{r}^{\tau}$ and (by transitivity) $\overline{\mathcal{I}}_{r}$.

Definition 3.28 ((class-based)). The domain ${ }^{k} \mathcal{I}_{r}$ is defined as the lattice

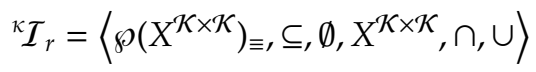

where (1) $X^{\mathcal{K} \times \mathcal{K}}$ is the set of all pairs $\left(\kappa_{1}, \kappa_{2}\right)$ s.t. $\kappa_{2}$ is reachable from $\kappa_{1}$; and (2) the equivalence relation is such that $S_{1} \equiv S_{2}$ are equivalent if they have the same downward closure w.r.t. $\leq$. Formally: let $S^{\leq}=\left\{\left(\kappa_{1}^{\prime}, \kappa_{2}^{\prime}\right) \mid \exists\left(\kappa_{1}, \kappa_{2}\right) \in S, \kappa_{1}^{\prime} \leq \kappa_{1}, \kappa_{2}^{\prime} \leq \kappa_{2}\right\}$, i.e., pairs obtained by adding all subclasses of classes belonging to a pair. Then $S_{1} \equiv S_{2}$ iff $S_{1}^{\leq}=S_{2}^{\leq}$. 
An abstract value ${ }^{1} T_{r}$ contains pairs of classes, and the intended meaning is that it represents all the states where a path goes from a $\kappa_{1}$ object to a $\kappa_{2}$ object only if $\left(\kappa_{1}, \kappa_{2}\right) \in{ }^{\mathcal{T}} T_{r}$. As discussed below, this is a very rough approximation of the concrete semantics. Due to how the equivalence relation is defined, abstract values can be considered as being closed on subclasses, like $S^{\leq}$.

Lemma 3.29. ${ }^{k} \mathcal{I}_{r}$ is an abstraction of $\mathcal{I}_{r}^{\tau}$.

It is straightforward to see that an abstract semantics based on ${ }^{k} \mathcal{I}_{r}$ and the corresponding ${ }^{K} \mathcal{I}_{c}$ would be much less precise than any other approaches to reachability/cyclicity analysis discussed in this paper: a variable of type $\kappa$ is condemned to be potentially cyclic as long as there is some possibility to create a cycle starting from a $\kappa$ object. On the other hand, the analysis is fully computable: it is only necessary to take the class hierarchy into account. This also implies that the reachability/cyclicity information does not depend on the program point: it can be computed once and used whenever needed. The interest of ${ }^{\kappa} \mathcal{I}_{c}$ is mainly theoretical because its lack of precision makes it impractical as the core of a static analyzer. However, it could be used as a first approximation which rules out some paths or cycles without the need of running more precise, but more expensive analyses like the other ones discussed here.

3.6.3. Abstract domains with restrictions on path-formulæ. The abstract domains introduced in this section are very similar to $\overline{\mathcal{I}}_{r}$, the only difference being the restriction of pathformulæ to some specific class of propositional formulæ. Domains ${ }^{\mathrm{P}} \mathcal{I}_{r}^{\tau},{ }^{\mathrm{M}} \mathcal{I}_{r}^{\tau}$, and ${ }^{\mathrm{D}} \mathcal{I}_{r}^{\tau}$ restrict path-formulæ to, respectively, positive, monotone, and definite Boolean functions (Section 3.1).

The domain ${ }^{\mathrm{P}} \mathcal{I}_{r}^{\tau}$ deals with positive Boolean functions with the addition of the bottom element false. The class of path-formulæ that can be represented includes monotone functions (note that the addition of false is needed to have this property), so that ${ }^{\mathrm{P}} \mathcal{I}_{r}^{\tau}$ can be easily proved to be a refinement of ${ }^{\mathrm{M}} \mathcal{I}_{r}^{\tau}$.

The restriction to monotone Boolean functions makes sense because a monotone function (with the exception of false, which is $p \wedge \neg p$ for some $p$, and true) can be represented by a conjunctive normal form where all literals are positive. In terms of paths and fields, a monotone formula can say that paths have to traverse a field, but not that they do not have to. Monotonicity implies that if a path $\pi$ p-satisfies a monotone path-formula $F$, then any path which contains $\pi$ as a part of it will also p-satisfy $F$.

Finally, the use of ${ }^{\mathrm{D}} \mathcal{I}_{r}^{\tau}$ can be motivated by the fact that, given a definite formula $F$, and two paths p-satisfying it and sharing a common part in the heap, their common part is guaranteed to p-satisfy $F$. For example, let $\mathcal{F}$ be $\{f, g, h\}$; in this case, the formula $g$ is definite. Consider the heap depicted below: both $\pi_{1}$ and $\pi_{2}$ p-satisfy $g$, and their intersection $\pi$ is also guaranteed to p-satisfy it.

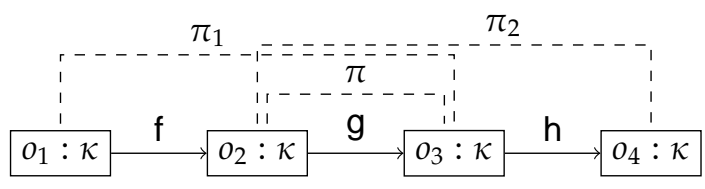

The rest of this section will formally define ${ }^{\mathrm{M}} \mathcal{I}_{r}^{\tau}$ and demonstrate that it is a strict abstraction of $\overline{\mathcal{I}}_{r}$; similar results can be also proved for ${ }^{\mathrm{P}} \mathcal{I}_{r}^{\tau}$ and ${ }^{\mathrm{D}} \mathcal{I}_{r}^{\tau}$.

Definition 3.30 ((monotone reachability)). The monotone field-reachability abstract domain is the complete lattice

$$
{ }^{\mathrm{M}} \mathcal{I}_{r}^{\tau}=\left\langle{ }^{\mathrm{M}} \mathcal{R}^{\tau},{ }^{\mathrm{M}} \unrhd_{r},{ }^{\mathrm{M}} \perp_{r},{ }^{\mathrm{M}} \mathrm{T}_{r},{ }^{\mathrm{M}} \sqcap_{r},{ }^{\mathrm{M}} \sqcup_{r}\right\rangle
$$


$-{ }^{n} \mathcal{R}^{\tau}$ is the set of functions from $\mathcal{X} \times \mathcal{X}$ to monotone path-formulæ, equipped with an equivalence relation similar to $\equiv^{v, w}$;

${ }^{\mathrm{M}}{ }_{r}$ is $\leq$ on path-formulæ, applied point-wise;

$-{ }^{\mathrm{M}_{\perp}}{ }_{r}=\lambda(v, w)$.false, and ${ }^{\mathrm{M}} \mathrm{T}_{r}=\lambda(v, w)$.true;

${ }^{\mathrm{M}}{ }^{\mathrm{M}} \eta_{r}$ is $\wedge$ applied point-wise, and ${ }^{\mathrm{M}} \sqcup_{r}$ is $\vee$ applied point-wise.

Lemma 3.31. The following abstraction and concretization functions define a Galois connection between $\overline{\mathcal{I}}_{r}$ and ${ }^{\mathrm{M}} \mathcal{I}_{r}^{\tau}$ : the latter strictly abstracts the former.

$$
\begin{aligned}
& \begin{cases}\text { false } & \text { if } \bar{I}_{r}(v, w) \models \text { false }\end{cases} \\
& \left(\alpha\left(\bar{I}_{r}\right)\right)(v, w)= \begin{cases}\text { true } & \text { if true } \equiv \bar{I}_{r}(v, w) \\
\bigwedge\left\{f l d_{1} \vee . . \vee \text { fld } \vec{k}_{k} \mid \bar{I}_{r}(v, w) \models f l d_{1} \vee . . \vee \text { fld } \vec{k}_{k}\right\} & \text { otherwise }\end{cases} \\
& \gamma\left({ }^{\mathrm{M}} \mathrm{I}_{r}\right)={ }^{\mathrm{M}} \mathrm{I}_{r}
\end{aligned}
$$

${ }^{\mathrm{M}} \mathcal{I}_{r}^{\tau}$ is strictly more abstract than $\overline{\mathcal{I}}_{r}$, as shown by the following example.

Example 3.32 ((monotone reachability)). Part (a) of the figure below shows a heap where $v$ can reach $w$ by traversing two paths.

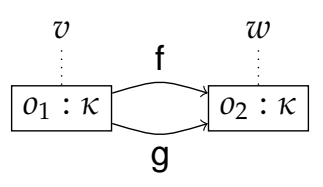

(a)

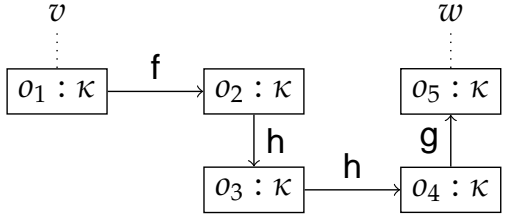

(b)

The abstract value which best represents such a heap in $\overline{\mathcal{I}}_{r}$ is $\bar{I}_{r}$ such that $\bar{I}_{r}(v, w)=\left(f^{\top} \mathrm{V}\right.$ $\left.g^{\prime}\right) \wedge\left(\neg f^{\wedge} \vee \neg g\right)\left(\right.$ exclusive disjunction). On the other hand, the best abstract value from ${ }^{\mathrm{M}} \mathcal{I}_{r}^{\tau}$ would be such that ${ }^{\mathrm{M}} I_{r}(v, w)=f^{\top} \vee g$. It can be easily seen that ${ }^{\mathrm{M}} I_{r}$ also represents heaps like part (b), where a path traverses both $f$ and $g$, whereas $\bar{I}_{r}$ does not.

As mentioned before, ${ }^{\mathrm{M}} \mathcal{I}_{r}^{\tau}$ is an abstraction of ${ }^{\mathrm{P}} \mathcal{I}_{r}^{\tau}$, while ${ }^{\mathrm{D}} \mathcal{I}_{r}^{\tau}$ can be compared with neither ${ }^{\mathrm{M}} \mathcal{I}_{r}^{\tau}$ nor ${ }^{\mathrm{P}} \mathcal{I}_{r}^{\tau}$ : for example, (1) $p \vee q$ is monotone but not definite, whereas $\neg p \vee q$ is definite but not monotone; and (2) $p \vee q$ is positive but, again, not definite, whereas $\neg p \wedge \neg q$ is definite but not positive.

3.6.4. An domain excluding fields from paths. The abstract domain introduced by [Scapin 2012], which will be denoted by $A_{\tau}$ in this paper, also considers field information to improve on existing techniques [Ghiya and Hendren 1996; Nikolic and Spoto 2014: Genaim and Zanardini 2013|. The property tracked by $A_{\tau}$ is "there are no paths from $v$ to $w$ which traverse any field belonging to a set $\mathrm{F}^{\prime \prime}$. The following definition is taken from [Scapin 2012, Def. 5.1], and slightly modified in order to adapt notation and only consider reachability.

Definition 3.33 ((Scapin's)). The complete lattice $\mathrm{A}_{\tau}$ is $\left\langle\mathrm{UR}_{\tau}, \sqsubseteq_{A}, \Pi_{A}, \sqcup_{A}\right\rangle$, where $\mathrm{UR}_{\tau}$ is $\wp(\mathcal{X} \times \mathcal{X} \times \wp(\mathcal{F})), \sqsubseteq_{A}$ is $\subseteq, \Pi_{A}$ is $\cap$, and $\sqcup_{A}$ is $\cup$.

An abstract value containing a triple $(v, w, \mathrm{~B})$, originally expressed as $v, \sim_{\rightarrow} \mathrm{B} w$, represents states where $v$ can only reach $w$ without traversing any fld $\in \mathrm{B}$.

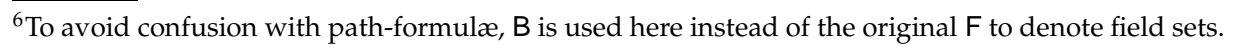


Lemma 3.34. The following functions define a Galois insertion between $\overline{\mathcal{I}}_{r}$ and $\mathrm{A}_{\tau}$ : the latter is a strict abstraction of the former.

$$
\begin{aligned}
& \alpha\left(\bar{I}_{r}\right)=\left\{v \not \rightarrow^{\mathrm{B}} w \mid \forall f l d \in \mathrm{B} . \bar{I}_{r}(v, w) \vDash \neg \text { flet }\right\} \\
& \gamma\left(I_{A}\right)=\lambda v, w . \bigwedge_{\text {fld } \in \mathrm{B}} \neg \text { flet } \quad \text { where } \mathrm{B} \text { is the maximal set s.t. } v \not \boldsymbol{B}^{\mathrm{B}} w \in I_{A}
\end{aligned}
$$

The abstract semantics and the complete analysis based on this domain is described by [Scapin 2012]. Importantly, it is not able to express the property that every cycle has to traverse certain fields, so that termination of the double-linked-list or the cyclic-tree example cannot be proved. However, the convergence of the global fixpoint is likely to be faster since $A_{\tau}$ only allows ascending chains of path-formulæ whose length is linear on the number of fields.

3.6.5. An analysis detecting that all paths have to traverse certain fields. The analysis presented by [Brockschmidt et al. 2012] uses some kind of field-sensitive information in order to prove termination. In fact, it is able to detect situations where all cycles which can occur in a data structure must traverse a certain set of fields, as in the example of Section 1.3 It is easy to see that such a piece of information, which is obtained by a component of their work, can be formalized into an abstract domain which is strictly less refined than ${ }^{\mathrm{M}} \mathcal{I}_{r}^{\tau}$. Unlike the other domains discussed in this section, the following definition refers to cyclicity instead of reachability since cyclicity is represented more explicitly by [Brockschmidt et al. 2012].

Definition 3.35. The complete lattice $Q_{\tau}$ is $\left\langle U_{\tau}, \sqsubseteq_{Q}, \Pi_{Q}, \sqcup_{Q}\right\rangle$, where $U_{\tau}$ is the set of partial functions from $\mathcal{X}$ to $\wp(\mathcal{F})$. An abstract value $I_{Q}$ represents concrete states where (1) for every $v \in \operatorname{dom}\left(I_{Q}\right)$ such that $I_{Q}(v)=\mathrm{B}, v$ can only be cyclic by means of paths which traverse all $f l d \in \mathrm{B}$; and (2) for every $w \notin \operatorname{dom}\left(I_{Q}\right)$, $w$ cannot be cyclic. Moreover,

$-I_{Q}^{1} \sqsubseteq_{Q} I_{Q}^{2}$ iff, for every $v \in \operatorname{dom}\left(I_{Q}^{1}\right)$, it holds that $v \in \operatorname{dom}\left(I_{Q}^{2}\right)$ and $I_{Q}^{2}(v) \subseteq I_{Q}^{1}(v)$ (i.e., $I_{Q}^{1}$ allows less variables to be cyclic and, in this case, puts stricter conditions on paths);

$-I_{Q}=I_{Q}^{1} \Pi_{Q} I_{Q}^{2}$ is such that $\operatorname{dom}\left(I_{Q}\right)=\operatorname{dom}\left(I_{Q}^{1}\right) \cap \operatorname{dom}\left(I_{Q}^{2}\right)$, and, for every $v \in \operatorname{dom}\left(I_{Q}^{1}\right) \cap$ $\operatorname{dom}\left(I_{Q}^{2}\right)$, it holds that $I_{Q}(v)=I_{Q}^{1}(v) \cup I_{Q}^{2}(v)$;

$-I_{Q}=I_{Q}^{1} \sqcup_{Q} I_{Q}^{2}$ is such that $\operatorname{dom}\left(I_{Q}\right)=\operatorname{dom}\left(I_{Q}^{1}\right) \cup \operatorname{dom}\left(I_{Q}^{2}\right)$, and (1) for every $v \in \operatorname{dom}\left(I_{Q}^{1}\right) \cap$ $\operatorname{dom}\left(I_{Q}^{2}\right)$, it holds that $I_{Q}(v)=I_{Q}^{1}(v) \cap I_{Q}^{2}(v) ;(2)$ for every $v \in \operatorname{dom}\left(I_{Q}^{1}\right) \backslash \operatorname{dom}\left(I_{Q}^{2}\right)$, it holds that $I_{Q}(v)=I_{Q}^{1}(v)$; and (3) for every $v \in \operatorname{dom}\left(I_{Q}^{2}\right) \backslash \operatorname{dom}\left(I_{Q}^{1}\right)$, it holds that $I_{Q}(v)=I_{Q}^{2}(v)$.

Lemma 3.36. The following functions define a Galois insertion between $\overline{\mathcal{I}}_{c}$ and $\mathrm{Q}_{\tau}$ : the latter is a strict abstraction of the former.

$$
\begin{aligned}
& \alpha\left(\bar{I}_{c}\right)= I_{Q} \text { with domain } D=\left\{v \mid \bar{I}_{c}(v) \neq \text { false }\right\} \text { and such that } \\
& I_{Q}(v)=\left\{\text { fld } \mid \bar{I}_{c}(v) \vDash \text { flet }\right\} \\
& \gamma\left(I_{Q}\right)=\lambda v \cdot \begin{cases}\bigwedge_{\text {fld } \in I_{Q}(v)} \text { flet } & \text { if } v \in \operatorname{dom}\left(I_{Q}\right) \\
\text { false } & \text { otherwise }\end{cases}
\end{aligned}
$$

As a matter of fact, $\mathrm{Q}_{\tau}$ is also an abstraction of the cyclicity counterpart of ${ }^{\mathrm{M}} \mathcal{I}_{r}^{\tau}$ since monotone boolean functions can capture the desired property. Indeed, the path formulæ returned by the function $\gamma$ presented in Lemma 3.36 (i.e., either false or $\bigwedge_{f l d \in I_{Q}(v)}$ flet $)$ are monotone.

3.6.6. Even more expressive abstract domains. Most domains discussed so far follow a similar pattern: an abstract value assigns to a pair of variables $(v, w)$ (or to a single variable, in the case of cyclicity) a logical formula which is in charge of describing a property of all 
paths between $v$ and $w$. This observation leads to consider more refined logics capturing finer-grained properties of paths.

For example, one could be interested in the order in which a path traverses fields. Such an order could be either a total or a partial order, stating that, for example, every path from $v$ to $w$ only traverses $f l d^{\prime \prime}$ after traversing $f l d^{\prime}$. Another potentially interesting property is the (minimum or maximum) number of occurrences of a given field in a path. In principle, these properties can be combined to represent even more precise properties such as all paths traverse fld' at least once, and fld" at least twice; the first occurrence of fld" comes before the first of $\mathrm{fld}^{\prime}$; the second occurrence of $\mathrm{fld} \mathrm{d}^{\prime \prime}$ comes after the first of fld'.

To define such domains and discuss their applicability is beyond the scope of this paper. Anyway, it is likely that this kind of properties of paths could be represented by first-order logic or some version of temporal logic or separation logic.

\section{THE FIELD-SENSITIVE ABSTRACT SEMANTICS}

This section defines an abstract semantics $\mathcal{Y}_{f l d}$ based on $\bar{I}_{r c}$. The semantics has to take into account any modification to the heap which may occur at runtime. In particular, paths can be created and removed by means of field updates. On the contrary, updating a reference variable (not one of its fields) does not modify the heap structure, but has to be reflected anyway in the resulting abstract values. An abstract denotation $\xi$ from $\tau_{1}$ to $\tau_{2}$ is a partial map from $\overline{\mathcal{I}}_{r c}^{\tau_{1}}$ to $\overline{\mathcal{I}}_{r c}^{\tau_{2}}$. It describes how the abstract input state changes when a piece of code is executed. The set of all abstract denotations from $\tau_{1}$ to $\tau_{2}$ is denoted by $\Xi\left(\tau_{1}, \tau_{2}\right)$. As in the concrete setting, interpretations provide abstract denotations for methods in terms of their input and output arguments. An interpretation $\zeta$ maps method signatures to abstract denotations, and is such that $\zeta(\mathrm{mth}) \in \Xi\left(\mathrm{mth}^{i}, \mathrm{mth}^{i} \cup\{\right.$ out $\left.\}\right)$ for every $\mathrm{mth}$. Note that the range of denotations is $\mathrm{mth}^{i} \cup\{$ out $\}$, unlike the concrete semantics where only out is needed since changes in the memory are directly observable in the heap. The set of all abstract interpretations is denoted by $\Psi$.

\subsection{Preliminaries}

4.1.1. Auxiliary analyses. $\boldsymbol{Y}_{\text {fld }}$ uses deep-sharing and purity [Genaim and Spoto 2008] analyses as pre-existent components; i.e., programs are assumed to have been analyzed w.r.t. these properties using state-of-the-art tools. Two reference variables $v$ and $w$ deepshare in $\sigma$ iff they both reach a common location by traversing non-empty paths, i.e., $R^{+}\left(\sigma^{h}, \sigma^{f}(v)\right) \cap R^{+}\left(\sigma^{h}, \sigma^{f}(w)\right) \neq \emptyset$, where $R^{+}(\cdot, \cdot)$ is like $R(\cdot, \cdot)$ but excludes empty paths. This property, written as $\langle v \backslash w\rangle$, is different from standard sharing [Secci and Spoto 2005] since paths from $\sigma^{f}(v)$ and $\sigma^{f}(w)$ to the common location must have length $\geq 1$. A variable deepshares with itself if the depth of the data structure pointed to by it is at least 2; the relation is symmetric.

Example 4.1 ((deep-sharing)). In the following heap, $x$ deep-shares with itself and with $y$; $y$ deep-shares with itself, with $x$, and with $z$; $z$ only deep shares with itself and with $y ; m_{1}$ and $\mathrm{m}_{2}$ alias but do not deep-share, not even with themselves.

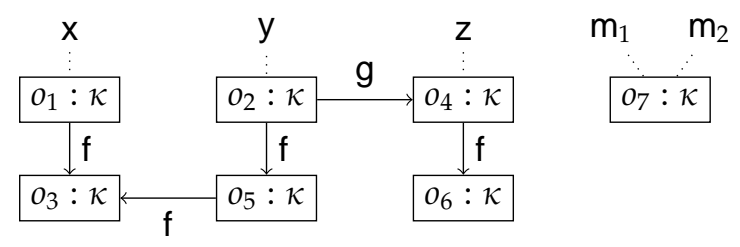

Note that two variables may deep-share without being reachable from each other, and one may reach the other without deep-sharing with it. This property is not exactly like $\downarrow$ of 
[Brockschmidt et al. 2012] since it requires both paths to have length $\geq 1$, not only one of them. However, it can be (and actually is, see Section (5) easily implemented as a variation of standard sharing analysis. Importantly, it is a possible analysis, i.e., a deep-sharing statement has to be added to the abstract description of the heap whenever there is the possibility of deep-sharing.

The $i$-th argument of a method mth is said to be pure if mth does not update the data structure to which the argument initially pointed. The analysis proposed by [Genaim and Spoto 2008], based on previous work by [Secci and Spoto 2005], can be used as purity analysis.

For each $\mathrm{mth}$, a denotation $\mathrm{SP}_{\mathrm{mth}}$ is given: for $I_{s p}\langle\mathrm{mth}\rangle$ safely describing the deep-sharing and purity between actual arguments in the input state, $I_{s p}^{\prime}\langle\mathrm{mth}\rangle=\mathrm{SP}_{\mathrm{mth}}\left(I_{s p}\langle\mathrm{mth}\rangle\right)$ is such that (1) if $\langle v \backslash w\rangle \in I_{s p}^{\prime}\langle\mathrm{mth}\rangle$, then $v$ and $w$ might become deep-sharing during the execution of mth; and (2) $\dot{v}_{i} \in I_{s p}^{\prime}\langle\mathrm{mth}\rangle$ means that the $i$-th argument might be impure. In the following, the domain $\mathcal{I}_{s p}^{\tau}$ will combine deep-sharing and purity information: $\langle v \backslash w\rangle \in I_{s p}$ means that $I_{s p}$ allows $v$ and $w$ to deep-share; and $\dot{v}_{i} \in I_{s p}$ means that $I_{s p}$ allows the $i$-th argument of the method under consideration to be impure.

4.1.2. Operations on abstract values. Projection $\exists v \bar{I}_{r c}$ (easily extensible to sets of variables) of $\bar{I}_{r c}$ sets $\bar{I}_{c}(v), \bar{I}_{r}(v, v)$, any $\bar{I}_{r}\left(w_{1}, v\right)$, and any $\bar{I}_{r}\left(v, w_{2}\right)$ to false, leaving the rest unchanged.

Renaming $\bar{I}_{r c}[v / w]$ replaces $v$ by $w$ : the result $\bar{I}_{r c}^{\prime}$ is such that $\bar{I}_{c}^{\prime}(w)=\bar{I}_{c}(v)$ and $\bar{I}_{c}^{\prime}(v)=$ false; $\bar{I}_{r}^{\prime}(v, v)=\bar{I}_{r}^{\prime}\left(v^{\prime}, v\right)=\bar{I}_{r}^{\prime}\left(v, v^{\prime}\right)=$ false for every $v^{\prime} ;$ moreover, $\bar{I}_{r}^{\prime}(w, w)=\bar{I}_{r}^{\prime}(v, v), \bar{I}_{r}^{\prime}\left(w, v^{\prime}\right)=\bar{I}_{r}\left(v, v^{\prime}\right)$ and $\bar{I}_{r}^{\prime}\left(v^{\prime}, w\right)=\bar{I}_{r}\left(v^{\prime}, v\right)$ for every $v^{\prime} \neq v$.

Copy $\bar{I}_{r c}[v+w]$ is similar to renaming but $v$ is not removed: the result $\bar{I}_{r c}^{\prime}$ is s.t.

$-\bar{I}_{c}^{\prime}(w)=\bar{I}_{c}^{\prime}(v)=\bar{I}_{c}(v)$;

$-\bar{I}_{r}^{\prime}(w, w)=\bar{I}_{r}^{\prime}(v, v)=\bar{I}_{r}(v, v)$ and $\bar{I}_{r}^{\prime}(v, w)=\bar{I}_{r}^{\prime}(w, v)=\bar{I}_{r}(v, v)$;

$-\bar{I}_{r}^{\prime}\left(w, v^{\prime}\right)=\bar{I}_{r}^{\prime}\left(v, v^{\prime}\right)=\bar{I}_{r}\left(v, v^{\prime}\right)$ and $\bar{I}_{r}^{\prime}\left(v^{\prime}, w\right)=\bar{I}_{r}^{\prime}\left(v^{\prime}, v\right)=\bar{I}_{r}\left(v^{\prime}, v\right)$ if $v^{\prime} \notin\{v, w\}$.

Finally, update $\bar{I}_{r c}[(v, w) \leftarrow F]$ sets $\bar{I}_{r}(v, w)$ to $F$, leaving the rest unchanged, and $\bar{I}_{r c}[v \leftarrow F]$ sets $\bar{I}_{c}(v)$ to $F$.

4.1.3. Path-formulæ. The path-concatenation operator $\odot: \mathcal{P F} \times \mathcal{P F} \mapsto \mathcal{P F}$ is used to combine formulæ when concatenating paths. The path-formula $F \odot G$ has the following models: $\left\{\omega^{\prime} \cup \omega^{\prime \prime} \mid \omega^{\prime} \in \operatorname{models}_{\mathcal{P}}(F) \wedge \omega^{\prime \prime} \in\right.$ modelsp $_{\mathcal{P}}(G) \wedge \omega^{\prime}$ and $\omega^{\prime \prime}$ are viable $\}$. In other words, the models of $F \odot G$ are obtained by "concatenating" the models of $F$ with those of $G$. This makes sense because of the following lemma.

Lemma 4.2 ((Path-CONCATENation)). Let $\pi^{\prime}$ and $\pi^{\prime \prime}$ be two paths such that the last location of $\pi^{\prime}$ is the first of $\pi^{\prime \prime}$. Then, $\pi^{\prime} \rightarrow F$ and $\pi^{\prime \prime} \rightarrow G$ imply $\pi^{\prime} \cdot \pi^{\prime \prime} \rightarrow F \odot G$.

It is easy to see that $\odot$ preserves equivalence of path-formulæ: if $F_{1} \equiv F_{2}$ and $G_{1} \equiv G_{2}$, then $F_{1} \odot F_{2} \equiv G_{1} \odot G_{2}$ since only viable models are considered.

The path-difference operator $\ominus: \mathcal{P F} \times \mathcal{P F} \mapsto \mathcal{P F}$ defines $\operatorname{models}_{\mathcal{P}}(F \ominus G)$ to be $\left\{\omega^{\prime} \backslash X \mid \omega^{\prime} \in\right.$ modelsp $(F) \wedge \omega^{\prime \prime} \in \operatorname{modelsp}(G) \wedge X \subseteq \omega^{\prime \prime} \wedge \omega^{\prime}$ and $\omega^{\prime \prime}$ are viable $\}$

Note that every model of $F$ is still a model of $F \ominus G$, since $\emptyset$ is a subset of all sets. The use of this operation is motivated by Lemmas 4.3 and 4.4. $\ominus$ models path difference.

Lemma 4.3. Let $\pi$ be $\pi^{\prime} \cdot \pi^{\prime \prime}$; let $\pi \rightarrow F$ and $\pi^{\prime} \rightarrow G$. Then, $\pi^{\prime \prime} \rightarrow F \ominus G$.

Lemma 4.4. Let $\pi$ be $\left\langle\ell_{0}, \ell_{1} . ., \ell_{k}\right\rangle$ and $\pi^{\prime}$ be $\left\langle\ell_{1}, . ., \ell_{k}\right\rangle$. Let the path from $\ell_{0}$ to $\ell_{1}$ traverse fld, and $\pi p$-satisfy $F$. Then, $\pi^{\prime} \rightarrow F \ominus\langle$ flet $\rangle$. 
$\left(1_{e}\right) \quad \mathcal{E}_{\zeta} \llbracket n \rrbracket\left(\bar{I}_{r c}\right)=\bar{I}_{r c}$

$\left(2_{e}\right) \quad \mathcal{E}_{\zeta} \llbracket$ null $\|\left(\bar{I}_{r c}\right)=\bar{I}_{r c}$

$\left(3_{e}\right) \quad \mathcal{E}_{\zeta} \llbracket$ new $\kappa \rrbracket\left(\bar{I}_{r c}\right)=\left(\bar{I}_{r}[(\rho, \rho) \leftarrow\langle\emptyset\rangle], \bar{I}_{c}[\rho \leftarrow\langle\emptyset\rangle]\right)$

$\left(4_{e}\right) \quad \mathcal{E}_{\zeta} \llbracket v \|\left(\bar{I}_{r c}\right)=$ if $\tau(v)=$ int then $\bar{I}_{r c}$ else $\bar{I}_{r c}[v+\rho]$

$\left(5_{e}\right) \mathcal{E}_{\zeta} \llbracket \exp _{1} \oplus \exp _{2} \rrbracket\left(\bar{I}_{r c}\right)=\exists \rho \cdot \mathcal{E}_{\zeta} \llbracket \exp _{2} \rrbracket\left(\exists \rho \cdot \mathcal{E}_{\zeta} \llbracket \exp _{1} \rrbracket\left(\bar{I}_{r c}\right)\right)$

$\left(6_{e}\right) \quad \mathcal{E}_{c} \llbracket$ Iv.fld $\rrbracket\left(\bar{I}_{r c}\right)=$ if fld has type int then $\bar{I}_{r c}$ else $\bar{I}_{r c} \overline{\bar{I}} \bar{I}_{r c}^{\prime}$ where

$$
\begin{aligned}
& \bar{I}_{c}^{\prime}(\rho)=\bar{I}_{c}(v) \\
& \bar{I}_{c}^{\prime}(w)=\text { false } \quad \text { for every } w \neq \rho \\
& \bar{I}_{r}^{\prime}(\rho, \rho)=\bar{I}_{c}(v) \\
& \bar{I}_{r}^{\prime}(\rho, w)=\bar{I}_{r}(v, w) \ominus\langle\text { flet }\rangle \quad \text { for every } w \neq \rho \\
& \bar{I}_{r}^{\prime}(w, \rho)= \begin{cases}\bar{I}_{r}(w, v) \odot\left\langle f l d^{2}\right\rangle & \text { if }\langle w \backslash v\rangle \notin I_{s p} \\
\text { true } & \text { if }\langle w\rangle v\rangle \in I_{s p}\end{cases} \\
& \bar{I}_{r}^{\prime}\left(w_{1}, w_{2}\right)=\text { false } \quad \text { in all the other cases }
\end{aligned}
$$

Fig. 2. The abstract semantics for expressions

$\left(7_{e}\right) \mathcal{E}_{\zeta} \llbracket\left[v_{0} \cdot m t h\left(v_{1}, . ., v_{n}\right) \rrbracket\left(\bar{I}_{r c}\right)=\bar{I}_{r c} \bar{\square} \bar{I}_{r c}^{\prime \prime} \bar{\square} \bar{I}_{r c}^{\prime \prime \prime} \overline{I_{r c}^{\prime \prime \prime}} \bar{I}_{r c}^{\prime \prime \prime}\right.$ where $\bar{v}=\left\{v_{0}, . ., v_{n}\right\}$ and

$\bar{I}_{r c}^{\prime}=\exists(\tau \backslash \bar{v}) \cdot \bar{I}_{r c} \quad I_{s p}^{\prime}=\exists(\tau \backslash \bar{v}) \cdot I_{s p}$

$\bar{I}_{r c}^{\prime \prime}=\bar{\square}\left\{\left(\zeta(\mathrm{mth})\left(\bar{I}_{r}^{\prime}\left[\bar{v} / \mathrm{mth}^{i}\right]\right)\right)\left[\mathrm{mth}^{i} / \bar{v}\right.\right.$, out $\left./ \rho\right] \mid \mathrm{mth}$ can be called here $\}$

$I_{s p}^{\prime \prime}=\cup\left\{\mathrm{SP}_{\mathrm{mth}}\left(I_{s p}^{\prime}\left[\bar{v} / \mathrm{mth}^{i}\right]\right)\left[\mathrm{mth}^{i} / \bar{v}\right.\right.$, out $\left./ \rho\right] \mid \mathrm{mth}$ can be called here $\}$

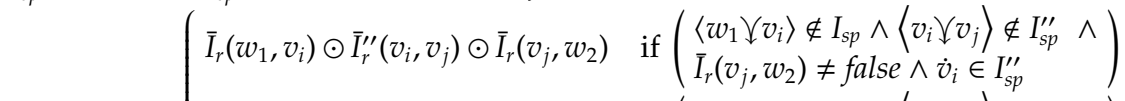



$$
\begin{aligned}
& \bar{I}_{r}^{\prime \prime \prime}=\bar{I}\left\{I_{r}^{i j} \mid i, j \in\{1 . . n\}\right\}
\end{aligned}
$$$$
\bar{I}_{r}^{\prime \prime \prime \prime}=\bar{I}_{r}^{\prime \prime \prime}\left[(\rho, w) \leftarrow \vee_{0 \leq i \leq n} F_{i}\right] \quad \text { for each } w
$$$$
\text { where } F_{k}= \begin{cases}\text { true } & \text { if }\left\langle v_{k} \backslash \rho\right\rangle \in I_{s p}^{\prime \prime} \\ \left(\bar{I}_{r}^{\prime \prime}\left(\rho, v_{k}\right) \odot \bar{I}_{r}\left(v_{k}, w\right)\right) \vee\left(\bar{I}_{r}\left(v_{k}, w\right) \ominus \bar{I}_{r}^{\prime \prime}\left(v_{k}, \rho\right)\right) & \text { otherwise }\end{cases}
$$$$
\bar{I}_{c}^{i}(w)= \begin{cases}\bar{I}_{c}^{\prime \prime}\left(v_{i}\right) & \text { if } \dot{v}_{i} \in I_{s p}^{\prime \prime} \wedge\left(\left\langle w \backslash v_{i}\right\rangle \in I_{s p} \vee \bar{I}_{r}\left(w, v_{i}\right) \neq \text { false } \vee \bar{I}_{r}\left(v_{i}, w\right) \neq \text { false }\right) \\ \text { false } & \text { otherwise }\end{cases}
$$$$
\bar{I}_{c}^{\prime \prime \prime}=\square\left\{\bar{I}_{c}^{i} \mid i \in\{1 . . n\}\right\}
$$$$
\bar{I}_{c}^{\prime \prime \prime \prime}=\bar{I}_{c}^{\prime \prime \prime}\left[\rho \leftarrow \bigvee\left\{\bar{I}_{c}\left(v_{k}\right) \mid 0 \leq k \leq n, \bar{I}_{r}^{\prime \prime}\left(v_{k}, \rho\right) \neq \text { false }\right\}\right]
$$

Fig. 3. The abstract semantics for method calls

\subsection{Expressions}

Figures 2 and 3 describe how the abstract semantics $\mathcal{E}_{\zeta} \llbracket$ I- $\rrbracket$ works on expressions. It is based on a type environment $\tau$ (left implicit) and an interpretation $\zeta$ on methods. The special variable $\rho$ represents the result of evaluating the expression. It is easy to see that $\mathrm{p}$-formulæ which are not in normal form are never generated.

Easy cases. As expected, the evaluation of an int value (case $1_{e}$ ) or null (case $2_{e}$ ) does not modify the current abstract value (i.e., there is no new reachability/cyclicity) since $\rho$ will not have any relation with any existing variable (either because it is a number or because it does not point to a valid heap location). When a new object is created (case $3_{e}$ ), the sharing information does change, but this is left implicit. More importantly, $\rho$ is correctly represented as (1) reaching itself through an empty path (only this kind of paths can p- 
satisfy $\langle\emptyset\rangle)$, which means that it aliases with itself; and (2) similarly, being cyclic because of an empty path.

In case $4_{e}$, information about $v$ is copied to $\rho$, without removing the original information as $v$ is still accessible. In case $5_{e}, \oplus$ stands for a binary operation on int; side effects are the only possible source of new information.

Field access. Case $6_{e}$ is harder: if the declared type of fld is a reference type, then the new abstract value is obtained by adding to the old one the following information.

- The cyclicity information about $v$ contained in $\bar{I}_{r c}$ affects $\bar{I}_{r c}^{\prime}$ in two ways: the path-formula is "copied" into both $\bar{I}_{c}^{\prime}(\rho)$ and $\bar{I}_{r}^{\prime}(\rho, \rho)$ indicating that the cyclicity of $v$ implies that (1) the data structure reachable from $v$.fld is still possibly cyclic; and (2) it is also possible that the location pointed to by $v$.fld is part of the cycle, so that it can be reachable from itself. The corresponding path-formula is copied as it is: the new path-formula $\bar{I}_{c}^{\prime}(\rho)=\bar{I}_{r}^{\prime}(\rho, \rho)$ is not greater than $\vec{I}_{c}(v)$ because the set of cycles reachable from $v$.fld is a subset of those reachable from $v$, so that they will satisfy the same condition (i.e., p-satisfy the same path-formula); on the other hand, it cannot be smaller because, by soundness, it is not possible to refine the condition. Note that the definition also works if $\bar{I}_{c}(v)=$ false: in this case, $v$ was guaranteed to be acyclic, so that $\rho$ is still guaranteed to be acyclic.

- The cyclicity of all the other variables is not modified: $\bar{I}_{c}^{\prime}(w)=$ false implies that the final cyclicity information $\bar{I}_{c}(w) \bar{\Xi}_{c} \bar{I}_{c}^{\prime}(w)$ for any $w$ is still the old $\bar{I}_{c}(w)$.

- If $v$ can $F$-reach some $w$ in $\bar{I}_{r c}$, then $v$.fld can also reach $w$ since it could be exactly on the path from $v$ to $w$. Therefore, $\bar{I}_{r}^{\prime}(\rho, w)$ is set to $F^{\prime}$ where $F^{\prime}$ is obtained from $\bar{I}_{r}(v, w)$ via $\ominus$ (Section 4.1.3). This means that it is no longer possible to guarantee that fld will be traversed by a path from $\sigma^{f}(\rho)$ to $\sigma^{f}(w)$.

- Every $w$ possibly deep-sharing with $v$ may reach $\rho$. In fact, deep-sharing means that there is a location which is reachable from both, and such a location could be exactly the one pointed to by $\rho$. In this case, the corresponding path-formula is true because it is not possible to put any condition on paths (deep-sharing as it is used by $\boldsymbol{y}_{\text {fld }}$ is fieldinsensitive). On the contrary, if $w$ and $v$ do not deep-share, then the following information is added: if $w F$-reaches $v$, then $\bar{I}_{r}^{\prime}(w, \rho)$ is set to $F \odot\langle f l d\rangle$, indicating that any new path from $\sigma^{f}(w)$ to $\sigma^{f}(\rho)$ will traverse fld (old paths are already accounted for by $\bar{I}_{r}$ ). Note that false $\odot F=$ false, so that any $w$ not reaching $v$ will not reach $\rho$, as expected.

Example 4.5 ((field access)). Consider the heap depicted in the right-hand side of the figure, which is the result of executing the program on the left-hand side.


Let the abstract value $\bar{I}_{r c}^{7}$ computed by the analysis after line 7 be such that $\bar{I}_{r}^{7}(\mathrm{x}, \mathrm{z})=\left\langle\mathrm{f}^{\mathrm{r}}, \mathrm{h}^{\top}\right\rangle$ (the computation of such an abstract value will be explained in Example 4.8). The new path-formula $\bar{I}_{r}^{8}(\mathrm{x}, \mathrm{z})$ after line 8 is obtained by $\ominus$ : first, $\bar{I}_{r}^{7}(\rho, \mathrm{z})$ is updated with $\bar{I}_{r}^{7}(\mathrm{x}, \mathrm{z}) \ominus\left\langle f^{\prime}\right\rangle$; afterward (see case $2_{c}$ in Figure 4), the new path-formula is copied to $\bar{I}_{r}^{8}(\mathrm{x}, \mathrm{z})$. The final path-formula will be $h^{\top} \wedge \wedge\left\{\neg f^{\prime} \mid f l d \notin\{f, h\}\right\}$ since it is no longer possible to guarantee that all paths from $x$ to $z$ traverse $f$. Anyway, note that $f$ does not appear as a negative literal either, as it is still possible that some path traverses it. 
Method call. Finally, case $7_{e}$ in Figure 3 describes the behavior of $\boldsymbol{y}_{\text {fld }}$ on method calls. Note that methods without return value are not included in the language; however, they could be easily dealt with by slightly modifying this case. As usual in Object-Oriented programs, a reference variable $v$ with declared type $\delta(v)=\kappa$ may store at runtime any object of type $\kappa^{\prime} \leq \kappa$. The set of possible runtime types of $v$ can be computed statically by class analysis [Spoto and Jensen 2003] whenever needed; if such an analysis is not available, then it can be taken, conservatively, as $\{\kappa \mid \kappa \leq \delta(v)\}$. Abstract values $\bar{I}_{r c}^{\prime}$ and $I_{s p}^{\prime}$ are obtained by restricting the corresponding initial values to the actual parameters $\bar{v}$ of $m t h$. Also, $\bar{I}_{r c}^{\prime \prime}$ and $I_{s p}^{\prime \prime}$ come from applying the denotation of mth for, resp., reachability/cyclicity (see Section 4.4) and deep-sharing/purity (which is taken as pre-computed information, see Section 4.1.1).

For every two actual parameters $v_{i}$ and $v_{j}$, the non-purity of $v_{i}$ implies that it is possible to create a path in mth from $v_{i}$ (or any $w_{1}$ reaching it or deep-sharing with it) to $v_{j}$ (or any $w_{2}$ reachable from it). This is taken into account by $\bar{I}_{r}^{i j}$ (note that there is an abstract value $\bar{I}_{r}^{i j}$ for every pair of parameters $\left(v_{i}, v_{j}\right)$, and all the values are combined into $\left.\bar{I}_{r}^{\prime \prime \prime}\right)$, and happens because modifying the data structure pointed to by $v_{i}$ during the execution of $m t h$ can possibly create a new path from $w_{1}$ to $w_{2}$. Four cases (plus an "otherwise" fifth case) are considered, depending on whether deep-sharing between $w_{1}$ and $v_{i}$ before the call or between $v_{i}$ and $v_{j}$ after the call is possible; all cases apply only if $v_{j}$ may reach $w_{2} \mathrm{~V}^{7}$ and $v_{i}$ is not pure. Basically, each of the four cases deals with the different scenarios with respect to deep-sharing: since this property is not field-sensitive, some field information about paths is lost whenever two variables may deep-share, since there can be complex paths in the heap for which no field-sensitive information is available (and which may be hidden from reachability analysis).

- The first case models a scenario where all new paths from $w_{1}$ to $v_{i}$ and from $v_{i}$ to $v_{j}$ are captured by reachability path-formulæ, so that it is possible to say that any path from $w_{1}$ to $w_{2}$ must traverse a sub-path captured by $\bar{I}_{r}\left(w_{1}, v_{i}\right)$, then another sub-path captured by $\bar{I}_{r}^{\prime \prime}\left(v_{i}, v_{j}\right)$ and, finally a third one captured by $\bar{I}_{r}\left(v_{j}, w_{2}\right)$. In order to account for this situation, the path-formula $\bar{I}_{r}\left(w_{1}, v_{i}\right) \odot \bar{I}_{r}^{\prime \prime}\left(v_{i}, v_{j}\right) \odot \bar{I}_{r}\left(v_{j}, w_{2}\right)$ is returned.

- The second case models a partial loss of information due to possible deep-sharing between $v_{i}$ and $v_{j}$. In this case, it is still possible to say that the first part of any new path from $w_{1}$ to $w_{2}$ is captured by $\bar{I}_{r}\left(w_{1}, v_{i}\right)$, but nothing can be inferred about the rest of the path.

- The third case is dual: here, the loss of information occurs in the first part of the path, since there is no field-sensitive information about paths starting from $w_{1}$ and reaching $v_{i}$.

- Finally, the fourth case happens when deep-sharing is possible both between $w_{1}$ and $v_{i}$ and between $v_{i}$ and $v_{j}$. In this case, reachability from $w_{1}$ to $w_{2}$ must be admitted as a possibility, but no field-sensitive information can be gathered, so that true has to be returned.

The following example describes the loss of information due to deep-sharing.

Example 4.6 ((method call)). Consider the figure below (only solid lines): let $v_{i}$ and $v_{j}$ be parameters of $m t h$ which are initially not sharing; let $w_{1}$ (which is not a parameter) deep-share with $v_{i}$, and $v_{j}$ reach $w_{2}$.

\footnotetext{
${ }^{7}$ Actually, in the first and third case there is no need of such a condition since false is the absorbing element of $\odot$; for instance, in the first case, $\bar{I}_{r}\left(w_{1}, v_{i}\right) \odot \bar{I}_{r}^{\prime \prime}\left(v_{i}, v_{j}\right) \odot$ false comes to be false anyway.
} 


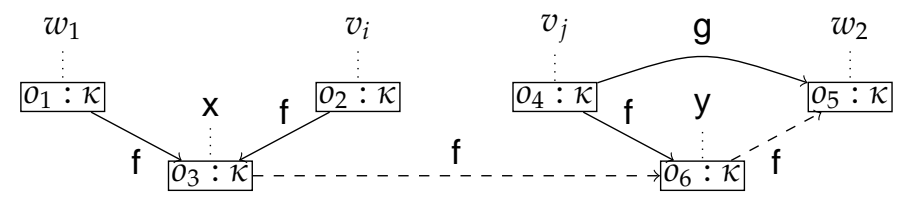

If the instructions $\mathrm{x}:=v_{i} . \mathrm{f} ; \mathrm{y}:=v_{j} . \mathrm{f} ; \mathrm{x} . \mathrm{f}:=\mathrm{y}$ are executed in $m t h$, then $v_{i}$ and $v_{j}$ become deep-


lines in the figure above. Note that (1) the new deep-sharing between $v_{i}$ and $v_{j}$ is reflected by the denotation of $m t h$; (2) there is never any reachability between $v_{i}$ and $v_{j}$, so that field-sensitive information is not available outside $m$ th (i.e., when applying its denotation); and (3) there is no way to create the path unless $v_{j}$ is reaching $w_{2}$. This situation falls into the fourth case of the semantics, so that true is returned as the reachability from $w_{1}$ to $w_{2}$.

Note that, in the example, $o_{3}$ happens to be a cutpoint [Rinetzky et al. 2005], i.e., an object which is (a) reachable from a parameter of $m t h$ in at least one step; and (b) also reachable by traversing a path which does not include any object which is reachable from any parameter of $m t h$. The existence of a cutpoint is possible in the third and fourth case above, where $\left\langle w_{1} \bigvee v_{i}\right\rangle \in I_{s p}$. Techniques similar to [Rinetzky et al. 2005] can be used to deal with such cases; alternatively, the analysis could be limited to cutpoint-free programs [Kreiker et al. 2013], since cutpoint-freeness is a decidable property.

Observation (3) in Example 4.6 shows that the reachability between two variables $w_{1}$ and $w_{2}$ will be certainly taken into account by some of the $\bar{I}_{r}^{i j}$, namely, the ones where $v_{i}$ and $v_{j}$ become sharing (in the normal sense, not deep-sharing), and $w_{1}$ shares with $v_{i}$, and $v_{j}$ reaches $w_{2}$.

The next step is to propagate the information about some $v_{i}$ in $\bar{I}_{r}^{\prime \prime \prime}$ to $\rho$ whenever $v_{i}$ (standard-)shares with $\rho$ after the call. This is needed in order to take into account some cases similar to Example 4.7 .

Example 4.7 ((return value)). Consider the following fragment:

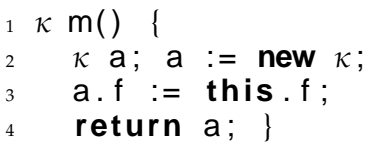

The abstract semantics computes the formula $F=\left\langle f^{\prime}, g\right\rangle$ for the reachability from $\times 2$ to $y$ after line 6 . After line $7, z$ should be reaching $y$, but this cannot be taken into account by the denotation of $m$ since $y$ is not a parameter. In fact, the denotation of $m$ can only detect that $\rho$ and this share at the end of $m$, i.e., that $z$ and $x 2$ share after the call. The only way to be sound here (note that this is pure, so that this situation is not detected by any $\bar{I}_{r}^{i j}$ ) is to copy the reachability from $\mathrm{x} 2$ to $\mathrm{y}$ to the reachability from $\rho$ to $\mathrm{y}$, which is in turn copied into $\bar{I}_{r}(\mathrm{z}, \mathrm{y})$. However, this is still unsound because the condition on paths could change: the two cases for $F_{k}$ in the abstract semantics account for the different scenarios: (1) if $\rho$ reaches this, then the new path is obtained by using $\odot$; (2) if this reaches $\rho$, then $\ominus$ is used; or (3) if they only deep-share, as in the example, then no information can be gathered, and true is returned.

As for cyclicity, each $\bar{I}_{c}^{i}$ deals with cases where a cycle is built in $m$ th which is reachable from $v_{i}$, and $w$ was sharing 8 with $v_{i}$; in this case, $w$ also becomes possibly cyclic. The rest of the treatment of cyclicity is similar to reachability.

${ }^{8}$ Note that standard sharing is the disjunction between deep-sharing and both directions of reachability. 


$$
\begin{aligned}
& C_{\zeta} \llbracket \mathbf{s k i p} \rrbracket\left(\bar{I}_{r c}\right)=\bar{I}_{r c} \\
& C_{\zeta} \llbracket v:=\exp \rrbracket\left(\bar{I}_{r c}\right)=\left(\exists v \cdot \mathcal{E}_{\zeta} \llbracket \exp \rrbracket\left(\bar{I}_{r c}\right)\right)[\rho / v] \\
& C_{c} \llbracket v \cdot f l d:=\exp \rrbracket\left(\bar{I}_{r c}\right)=\exists \rho \cdot\left(\bar{I}_{r c}^{\prime} \sqcup \overline{I_{r}^{\prime}} \bar{I}_{r}^{\prime} \overline{I_{c}^{\prime \prime}}\right) \text { where } \\
& \bar{I}_{r c}^{\prime}=\mathcal{E}_{c} \llbracket \exp \rrbracket\left(\bar{I}_{r c}\right) \\
& \left.\bar{I}_{r}^{\prime \prime}\left(w_{1}, w_{2}\right)=\bar{I}_{r}^{\prime}\left(w_{1}, v\right) \odot\left(\langle\text { flet }\rangle \vee(\langle f \mid l d\rangle) \odot \bar{I}_{r}^{\prime}(\rho, v)\right)\right) \odot \bar{I}_{r}^{\prime}\left(\rho, w_{2}\right) \\
& \bar{I}_{c}^{\prime \prime}(w)= \begin{cases}\left(\bar{I}_{r}^{\prime}(\rho, v) \odot\langle\text { flet }\rangle\right) \vee \bar{I}_{c}(\rho) & \text { if } \bar{I}_{r}^{\prime}(w, v) \neq \text { false } \\
\text { false } & \text { otherwise }\end{cases} \\
& \bar{I}_{r c}^{1}=C_{\zeta} \llbracket c o m_{1} \rrbracket\left(\bar{I}_{r c}\right) \\
& \bar{I}_{r c}^{2}=C_{\zeta} \llbracket \operatorname{com}_{2} \rrbracket\left(\bar{I}_{r c}\right)
\end{aligned}
$$

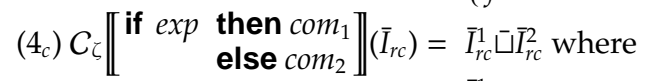

(5 $\left.5_{c}\right) \mathcal{C}_{\zeta} \llbracket$ while $\exp$ do $\operatorname{com} \rrbracket\left(\bar{I}_{r c}\right)=\xi\left(\bar{I}_{r c}\right)$ where $\xi$ is the least fixpoint of $\lambda w . \lambda \bar{I}_{r c} \cdot w\left(C_{\zeta} \llbracket \operatorname{com} \rrbracket\left(\bar{I}_{r c}\right)\right)$

(6) $\left.6_{c}\right) \quad C_{\zeta} \llbracket \operatorname{com}_{1} ; \operatorname{com}_{2} \rrbracket\left(\bar{I}_{r c}\right)=C_{\zeta} \llbracket \operatorname{com}_{2} \rrbracket\left(C_{\zeta} \llbracket \operatorname{com}_{1} \rrbracket\left(\bar{I}_{r c}\right)\right)$

(7c) $\quad C_{\zeta} \llbracket$ return $\exp \rrbracket\left(\bar{I}_{r c}\right)=\mathcal{E}_{\zeta} \llbracket \exp \rrbracket\left(\bar{I}_{r c}\right)[\rho /$ out $]$

Fig. 4. The abstract semantics for commands

\subsection{Commands}

Figure 4 shows the behavior of the abstract semantics $C_{\zeta} \llbracket-\rrbracket$ on commands. Easy cases are considered first, leaving field update at the end.

Case $1_{c}$ is trivial. Case $2_{c}$ for variable assignment is also easy: the semantics evaluates exp, which could have side effects, and copies the information about $\rho$ to $v$, after removing the information about $v$ since its initial value will be lost. Note that the information about the location pointed to by $v$ needs not be lost, since there could be other variables pointing to it.

In cases $4_{c}$ and $5_{c}$, standard principles for the design of abstract semantics are followed.

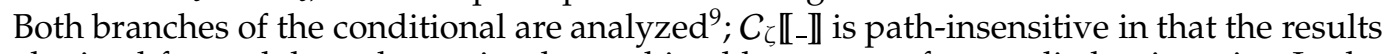
obtained for each branch are simply combined by means of $\vee$, applied point-wise. In the case of loops, standard fixpoint design is used. Termination of the fixpoint is guaranteed by the fact that $\overline{\mathcal{I}}_{r c}$ does not allow infinite ascending chains $\left\langle F_{0}, . ., F_{i}, ..\right\rangle$ where $F_{j} \underline{\underline{E}}_{r c} F_{j+1}$ for each $j \geq 0$. However, in principle, there can be chains whose length is exponential on the cardinality of $\mathcal{F}$, as discussed in Section 3.6, so that convergence can be slow unless some mechanism for speeding it up is used (e.g., some widening [Cousot and Cousot 1979] operator mapping path-formulæ to true whenever abstract values have been updated more than $k$ times for some fixed number $k$ ).

The last two cases, $6_{c}$ and $7_{c}$, are straightforward.

Field update. In $v$.fld:=exp, the heap is modified, and new paths can be created. In particular, a path $\pi$ from the location pointed to by $v$ to the location pointed to by $\rho$ is created, which p-satisfies flet? The information about $\pi$ must be joined with the original abstract value by means of $\bar{\square}$.

The abstract semantics focuses on two kinds of variables: the first kind, pre-variables, contains those $w_{1}$ which can reach $v$. The second kind of variables, post-variables, contains those $w_{2}$ which can be reached from $\rho$ after $\exp$ has been evaluated, and the abstract value $\bar{I}_{r c}^{\prime}$ has been computed. Clearly, $v$ is a pre-variable since it reaches itself. Moreover, a variable may belong to both kinds; in this case, it will be considered twice. The new reachability information $\bar{I}_{r}^{\prime \prime}$ must take into account paths from all pre-variables $w_{1}$ to all-post variables $w_{2}$, due to the creation of $\pi$. The new paths certainly p-satisfy $\bar{I}_{r}^{\prime}\left(w_{1}, v\right) \odot(\langle$ flet $\rangle \vee(\langle$ flet $\rangle \odot$ $\left.\left.\bar{I}_{r}^{\prime}(\rho, v)\right)\right) \odot \bar{I}_{r}^{\prime}\left(\rho, w_{2}\right)($ see Lemma 4.2).

\footnotetext{
${ }^{9}$ Recall that guards have no side effects.
} 
Note that both the newly-created path (represented by $\langle$ flet $\rangle$ ) and the possible cycle (represented by $\langle$ flet $\rangle \odot \bar{I}_{r}^{\prime}(\rho, v)$ ) which is created by the update are considered; this will become more clear when discussing the example in Section 4.7 Note also that this formula will not necessarily be the final reachability information about $w_{1}$ and $w_{2}$. In fact, suppose that there existed another path from $w_{1}$ to $w_{2}$, completely disjoint from the new one, and p-satisfying $G$ : in this case, the final reachability between $w_{1}$ and $w_{2}$ will be $G \vee\left(\bar{I}_{r}^{\prime}\left(w_{1}, v\right) \odot\right.$

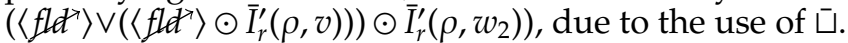

The cyclicity information comes from two cases: the definition of $\bar{I}_{c}^{\prime \prime}$ considers cycles created by closing existing paths from $\rho$ to $v$. Every $w$ reaching $v$ will become possibly cyclic, and the associated path-formula will be the combination of the old path with flet. Note that the conditions on the cyclicity of $w$ do not need to take into account $\bar{I}_{r}^{\prime}(w, v)$, since, in general, it is not in the cycle (if it is, then this information is already contained in $\bar{I}_{c}^{\prime}$ ).

Example 4.8 ((field update)). Consider the code of Example 4.5 it is easy to see that the abstract value $\bar{I}_{r c}^{5}$ after line 5 is such that $\bar{I}_{r}^{5}(\mathrm{x}, \mathrm{y})=\langle\neq\rangle$. Moreover, $\bar{I}_{r}^{5}(\mathrm{x}, \mathrm{x})=\bar{I}_{r}^{5}(\mathrm{y}, \mathrm{y})=$ $\bar{I}_{r}^{5}(\mathrm{z}, \mathrm{z})=\langle\emptyset\rangle$, and $\bar{I}_{r}^{5}\left({ }_{-},-\right)=$false everywhere else. Such a value is obtained by observing that neither $\mathrm{x}$ nor y reach any other variable before line 5 . Consequently, $\bar{I}_{r}^{5}(\mathrm{x}, \mathrm{y})$ comes to be $\langle\emptyset\rangle \odot\left\langle f^{\top}\right\rangle \odot\langle\emptyset\rangle=\left\langle f^{\top}\right\rangle$ since $\langle\emptyset\rangle$ is the neutral element for $\odot$. Similarly, $\bar{I}_{r c}^{6}(y, z)=\left\langle h^{\top}\right\rangle$. The path-formula $\bar{I}_{r c}^{6}(\mathrm{x}, \mathrm{z})=\bar{I}_{r c}^{7}(\mathrm{x}, \mathrm{z})$ is computed as $\left\langle f^{\top}\right\rangle \odot\left\langle\mathrm{h}^{\top}\right\rangle=\left\langle\boldsymbol{f}^{\top}, \mathrm{h}^{\top}\right\rangle$, and its only model is $\left\{f^{\top}, h^{\top}\right\}$.

\subsection{Global fixpoint}

The following definition defines the abstract denotational semantics of a program $P$ as the least fixpoint (lfp) of an (abstract) transformer of interpretations. Variables $\bar{u}$ play the role of shallow variables. Note that shallow variables appear at the level of the semantics, rather than as a result of program transformation; they are introduced in order to keep track of the data structures to which input variables point at the beginning of a method, since otherwise they could be lost if the corresponding variables are updated.

Example 4.9. Consider the following method mth (lines 1-4), invoked in line 5:



Here, the reachability from the first to the second parameter of mth after its execution would be lost if a copy of them is not maintained as a shallow variable. On the other hand, copying the information about $x 1$ and $\times 2$ into shallow variables $u_{1}$ and $u_{2}$ allows detecting that $u_{1}$ is reaching $u_{2}$ and $\rho$ at the end of mth. Afterward, this is copied back to actual parameters y1 and $y 1$ (line 5) in order to be available after the call.

Definition 4.10. The abstract denotational semantics of a program $P$ is the lfp of

$$
\mathcal{T}_{P}(\zeta)=\left\{\mathrm{mth} \mapsto \lambda \bar{I}_{r c} \in \mathcal{I}_{r c}^{\mathrm{mth}^{i}}\left(\exists X . C_{\zeta} \llbracket \mathrm{mth}^{b} \rrbracket\left(\bar{I}_{r c}[\bar{w}+\bar{u}]\right)\right)[\bar{u} / \bar{w}] \mid \mathrm{mth} \in P\right\}
$$

where $\mathrm{mth}^{i}=\left\{\right.$ this, $\left.w_{1}, \ldots, w_{n}\right\}$, and $\bar{u}$ is a variable set $\left\{u_{1}, \ldots, u_{n}\right\}$ such that $\bar{u} \cap \mathrm{mth}^{s}=\emptyset$; moreover, $\operatorname{dom}(\tau)=\mathrm{mth}^{l} \cup \bar{u}$, and $X=\operatorname{dom}(\tau) \backslash(\bar{u} \cup\{$ this, out $\})$.

The operator $\mathcal{T}_{P}$ is quite standard, and transforms the interpretation $\zeta$ by assigning a new denotation for each $\mathrm{mth}$ defined in $P$, using existing denotations from $\zeta$. The new denotation maps a given input value $\bar{I}_{r c} \in \bar{I}_{r c}^{\mathrm{mth}^{i}}$ to an output value from $\overline{\mathcal{I}}_{r c}^{\mathrm{mth}^{\mathrm{i}} \cup\{o u t\}}$, as follows: 
(1) it obtains an abstract value $\bar{I}_{r c}^{0}=\bar{I}_{r c}[\bar{w}+\bar{u}]$ in which the parameters $\bar{w}$ are cloned into the shallow variables $\bar{u}$;

(2) it applies the denotation of the code of mth to $\bar{I}_{r c}^{0}$, getting $\bar{I}_{r c}^{1}=C_{\zeta} \llbracket \llbracket \mathrm{mth}^{b} \rrbracket\left(\bar{I}_{r c}^{0}\right)$;

(3) all variables but $\bar{u} \cup\{$ this, out $\}$ are eliminated from $\bar{I}_{r c}^{1}$ (using $\exists X$ ); and

(4) shallow variables $\bar{u}$ are finally renamed back to $\bar{w}$.

\subsection{Soundness}

This section discusses the soundness of $\boldsymbol{y}_{\text {fld }}$. Consider a command C: soundness amounts to say that, for every initial state $\sigma$ correctly represented by the initial abstract value (i.e., such that $\left.\sigma \in \bar{\gamma}_{r c}\left(\bar{I}_{r c}\right)\right)$, the final concrete state $\sigma^{*}=C_{\tau}^{\iota} \llbracket C \rrbracket(\sigma)$ is such that $\sigma^{*} \in \bar{\gamma}_{r c}\left(\bar{I}_{r c}^{*}\right)$ where $\bar{I}_{r c}^{*}=C_{\zeta} \llbracket C \rrbracket\left(\bar{I}_{r c}\right)$. This means that every possible path from $w_{1}$ to $w_{2}$ created by $C$, and traversing a set $X$ of fields, has to be reflected by a model $\omega=\{$ flet $\mid$ fld $\in X\}$ of $\bar{I}_{r c}^{*}\left(w_{1}, w_{2}\right)$. A similar observation holds for cyclicity. Most of the evidence for soundness has been given while discussing the abstract semantics; this section summarizes and completes the proof. Only the most interesting cases are presented.

Field update, reachability. Consider a field update $v \cdot f:=v^{\prime}$. Given two variables $w_{1}$ and $w_{2}$, every path $\pi$ from $\sigma^{f}\left(w_{1}\right)$ to $\sigma^{f}\left(w_{2}\right)$ is either an old one (already in the heap before the update) or a newly-created one traversing $f$ at $\sigma^{f}(v)$. If $\pi$ was already in the heap, then, by hypothesis, it is represented by $\bar{I}_{r}$, and also by $\bar{I}_{r}^{*}$ since $\bar{I}_{r}^{*} \bar{\Xi}_{r} \bar{I}_{r}$. On the other hand, if $\pi$ is new, then it is the concatenation of the following sub-paths:

- a path $\pi_{1}$ from $\sigma^{f}\left(w_{1}\right)$ to $\sigma^{f}(v)$, which, by hypothesis, is correctly represented by the initial abstract value $\bar{I}_{r}\left(w_{1}, v\right)$;

- one of the following: either the path $\pi^{\prime}$ of length 1 going from $\sigma^{f}(v)$ to $\sigma^{f}\left(v^{\prime}\right)$ and psatisfying $\left\langle f^{\prime}\right\rangle$; or the path $\pi^{\prime \prime}$ going from $\sigma^{f}(v)$ to $\sigma^{f}\left(v^{\prime}\right)$, then to $\sigma^{f}(v)$ and back to $\sigma^{f}\left(v^{\prime}\right)$, and p-satisfying $\left\langle f^{\prime}\right\rangle \odot \bar{I}_{r}\left(v^{\prime}, v\right)$; and

- a path $\pi_{2}$ from $\sigma^{f}\left(v^{\prime}\right)$ to $\sigma^{f}\left(w_{2}\right)$, which is correctly represented by $\bar{I}_{r}\left(v^{\prime}, w_{2}\right)$.

The alternative of $\pi^{\prime \prime}$ has to be taken into account because it is possible that the field update closes a cycle, i.e., that there was already a path from $\sigma^{f}\left(v^{\prime}\right)$ to $\sigma^{f}(v)$ which has now become a cycle from $\sigma^{f}\left(v^{\prime}\right)$ to $\sigma^{f}\left(v^{\prime}\right)$. In this case, there is a path from $\sigma^{f}\left(w_{1}\right)$ to $\sigma^{f}\left(w_{2}\right)$ which goes until $\sigma^{f}(v)$ and $\sigma^{f}\left(v^{\prime}\right)$, then traverses the cycle until reaching $\sigma^{f}\left(v^{\prime}\right)$ again, and finally reaches $\sigma^{f}\left(w_{2}\right)$. Note that false is the absorbing element for $\odot$, so that $\pi^{\prime \prime}$ will be guaranteed to p-satisfy false (i.e., not to exist) if the initial information was able to exclude paths from $\sigma^{f}\left(v^{\prime}\right)$ to $\sigma^{f}(v)$ (i.e., if $\bar{I}_{r}\left(v^{\prime}, v\right)=$ false).

Example 4.11. Consider the following heap before executing $v . f:=v^{\prime}$ (which is the dashed line). After the field update, $w_{2}$ is reachable from $w_{1}$ either directly or "touching" $o_{5}$ any number of times. Note that a path touching $o_{5}$ once p-satisfies the same path-formula as one touching $o_{5}$ more than once. Reachability from $w_{1}$ to $w_{2}$ p-satisfies $\left\langle f^{\prime}\right\rangle \vee\left\langle f^{\prime}, g\right\rangle$.

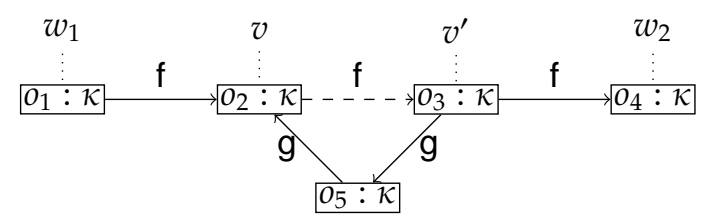

It is easy to see that both cases $\left(\pi^{\prime}\right.$ and $\left.\pi^{\prime \prime}\right)$ are dealt with by the definition of $\bar{I}_{r}^{\prime \prime}$ in case $\left(3_{c}\right)$ of Figure 4 This follows from the definition of $\odot$ and Lemma 4.2 .

It is worth noting that the field update could break some old paths. Conservatively, this is not taken into account by the semantics: the removal of a path from a heap can never 
imply that a path-formula is no longer p-satisfied, so that to update abstract values is not needed. More formally, it is possible to consider a field update as the combination of two operations: first set $v$.f to null, then assigning it to $v^{\prime}$. Let $\sigma$ and $\sigma^{-}$be two states such that the heap of $\sigma^{-}$is obtained by breaking (e.g., by setting some object fields to null) one or more paths in the heap of $\sigma$; then, $\sigma \in \bar{\gamma}_{r c}\left(\bar{I}_{r c}\right)$ implies $\sigma^{-} \in \bar{\gamma}_{r c}\left(\bar{I}_{r c}\right)$. This choice is sound but could lead, in some cases, to losing precision. A possible improvement (already discussed by [Genaim and Zanardini 2013] for $\boldsymbol{Y}_{r c}$ ) would be to distinguish cases where the class of $v$ only declares the field $f$; in this case, to update $f$ would be guaranteed to break all existing paths starting from $\sigma^{f}(v)$, and this information could be used in order to set all $I_{r c}(v, w)$ to false for every $w$ before computing the final $\bar{I}_{r c}^{*}$.

Field update, cyclicity. As for cyclicity, the initial information $\bar{I}_{c}(w)$ about a variable $w$ is updated only if $w$ can possibly reach $v$. In fact, new cycles reachable from $w$ can appear only if (1) an existing cycle is made reachable from $v$ by the field update; or (2) a new cycle is created which touches both $\sigma^{f}(v)$ and $\sigma^{f}\left(v^{\prime}\right)$. In both cases, the cycle under study will not be reachable from $w$ unless $v$ is reachable from $w$ : (1) in the first case, because the new path from $w$ to the cycle has to touch $\sigma^{f}(v)$; and (2) in the second case, because a variable reaching a cycle must reach every single heap location belonging to the cycle itself.

Example 4.12. Consider the following heap before the field update (dashed line): the cycle which will be created will not be reachable from $w$, even though it deep-shares with (but does not reach) $v$.



On the other hand, reachability from $w$ to $v$ implies that new cycles can become reachable from $w$. The path-formula $\left(\bar{I}_{r}\left(v^{\prime}, v\right) \odot\left\langle f^{\prime}\right\rangle\right) \vee \bar{I}_{c}\left(v^{\prime}\right)$, where $v^{\prime}$ plays the role of $\rho$, accounts exactly for both kinds of loops discussed above: (1) newly-created loops p-satisfying $\bar{I}_{r}\left(v^{\prime}, v\right) \odot\langle f\rangle$; and (2) existing loops which were reachable from $v^{\prime}$ and now are reachable from $v$ and (by transitivity) from $w$.

Method call, reachability. Consider a method call $v:=v_{0} \cdot \operatorname{mth}\left(v_{1} . . v_{k}\right)$, and two variables $w_{1}$ and $w_{2}$ which are visible from the caller. Let the interest be on the reachability from $w_{1}$ to $w_{2}$ after executing $\mathrm{mth}$. Let also $M_{i}$ be the portion of the heap which is reachable from some $v_{i}$ : it is clear that nothing will be modified by mth outside $M=M_{0} \cup . . \cup M_{k}$.

The path-formula describing paths from $w_{1}$ to $w_{2}$ which are possibly created inside mth depends on the position of $\ell_{1}=\sigma^{f}\left(w_{1}\right)$ and $\ell_{2}=\sigma^{f}\left(w_{2}\right)$ w.r.t. $v_{0} . . v_{k}$ and $M_{0} . . M_{k}$. The abstract semantics analyzes the position of $\ell_{1}$ and $\ell_{2}$ with respect to each pair $\left(v_{i}, v_{j}\right)$ separately, and joins the results together. The definition of $\bar{I}_{r}^{i j}\left(w_{1}, w_{2}\right)$ in Figure 3$)$, consists of five cases, named (a), (b), (c), (d), and (e) in the following. If $\ell_{2}$ does not belong to $M_{j}$, then no path can be created which reaches it, and the abstract semantics correctly returns false (case (e)). Also, $v_{i}$ must not be pure, otherwise there is no way to create new paths in mth. Cases (a), (b), (c), and (d) capture the different scenarios w.r.t. $\ell_{1}, v_{i}$ and $v_{j}$.

- If $\ell_{1}$ reaches $v_{i}$, but does not deep-share with it, and $v_{i}$ does not deep-share with $v_{j}$ after the call, then the information is completely captured by reachability abstract values, i.e., no field-sensitive information is lost. The formula returned in case (a) correctly represent the reachability between $w_{1}$ and $w_{2}$ since new paths will touch $\sigma^{f}\left(v_{i}\right)$ (there can be new paths not touching $\sigma^{f}\left(v_{i}\right)$, but they will be captured by some different $\left.\bar{I}_{r}^{i^{\prime} j^{\prime}}\right)$. Note that 
a new path does not need to touch any other parameter than $v_{i}$; in this case, it will be accounted for, at least, when $j$ coincides with $i$. Note also that false is the absorbing element for $\odot$, so that non-reachability between $w_{1}$ and $v_{i}$, or between $v_{i}$ and $v_{j}$, or between $v_{j}$ and $w_{2}$, implies that no path is actually created.

- If $\ell_{1}$ reaches $v_{i}$, but does not deep-share with it, and $v_{i}$ may deep-share with $v_{j}$, then some field-sensitive information may be lost after mth. This is reflected by case (b), where the second part of the formula is set to true. The first part is still the reachability from $w_{1}$ to $v_{i}$.

- If $\ell_{1}$ deep-shares with $v_{i}$, and $v_{i}$ does not deep-share with $v_{j}$, then, again, some fieldsensitive information may be lost after mth. This is accounted for by case (c), where the first part of the formula is true. On the other hand, the second part is the reachability from $v_{j}$ to $w_{2}$ since it is possible to guarantee that the last part of the new path will follow reachability paths between these two variables.

- Case $(\mathrm{d})$ is trivially sound since true is returned.

Method call, cyclicity. Consider, again, the method call $v:=v_{0} \cdot \operatorname{mth}\left(v_{1} . . v_{k}\right)$ and a variable $w$ of the caller. Suppose the interest is on the cyclicity of $w$ after executing mth. Again, the portion $M=M_{0} \cup . . \cup M_{k}$ of the heap which can be affected by the execution of mth is the set of locations which are reachable from some of the $v_{i}$ in zero or more steps. The location $\ell=\sigma^{f}(w)$ can fall into one of the following cases:

$-\ell \notin M_{i}$ and no location of $M_{i}$ is reachable from $\ell$; in this case, no new cycles will be reachable from $w$, and there is nothing to prove.

$-\ell \notin M_{i}$ and some $v_{i}$ is reachable from it; in this case, suppose that a new cycle is created in the heap by the execution of $\mathrm{mth}$, which is reachable from $v_{i}$ : soundness requires that the cycle has to be also reachable from $w$ after the call. This is satisfied by the abstract semantics since:

$-v_{i}$ is impure (otherwise, no new cycle can be created); and

- there is reachability from $w$ to $v_{i}$, by hypothesis.

Under these circumstances, the path-formula returned for the cyclicity of $w$ will be $\bar{I}_{c}^{\prime \prime}\left(v_{i}\right)$, which is correct since every cycle reachable from $v_{i}$ will be also inferred to be reachable from $w$.

$-\ell \in M_{i}$ : in this case, $\ell$ is reachable from $v_{i}$ and, again, the path-formula $\bar{I}_{c}^{\prime \prime}\left(v_{i}\right)$ is a correct description of cycles reachable from $w$. In fact, $\bar{I}_{c}^{i}(w)$ is not greater than $\bar{I}_{c}^{\prime \prime}\left(v_{i}\right)$ since the portion of the heap which is reachable from $w$ is a subset of the portion reachable from $v_{i}$, so that the same path-formula is certainly satisfied; on the other hand, it cannot be smaller because, in general, it is not possible to identify any cycle reachable from $v_{i}$ which is not reachable from $w$.

$-\ell \notin M_{i}$ and $\left\langle w \backslash v_{i}\right\rangle$; in this case, the first location reachable from both happens to be a cutpoint [Rinetzky et al. 2005|. Anyway, cycles reachable from $w$ are still the same cycles reachable trom $v_{i}$ (this is different w.r.t. reachability because path-formulæ for cyclicity ignore the acyclic part of the path).

\subsection{Back to the cyclic-tree example}

Consider the code of Section 1.2. Suppose that the input parameters of join are represented by the abstract values depicted on the right-hand side of the figure, probably inferred by previous steps of the analysis. Different input abstract values would yield comparable results. 


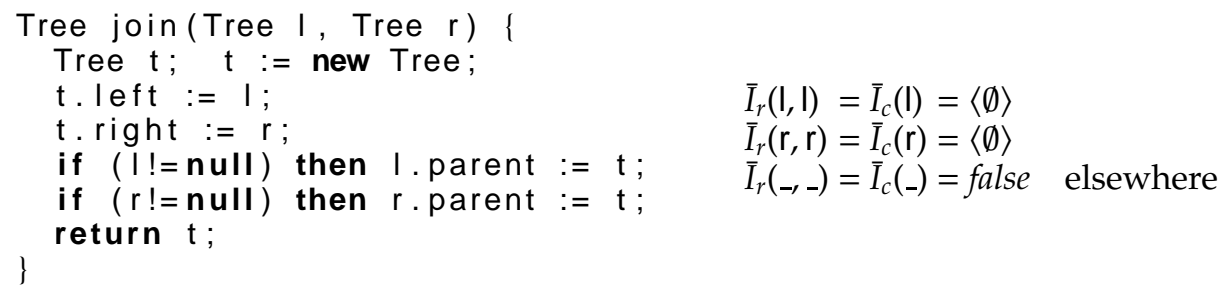

This information indicates that both parameters, which will be the sub-trees of the tree created by join, represent trees of at most one node (they could be also null pointers representing 0 -node trees, since the previous analysis could have been overly conservative). Next tables show the result of the analysis. $\bar{I}_{r c}^{i}=\left(\bar{I}_{r}^{i}, \bar{I}_{c}^{i}\right)$ is the abstract value computed after line $i$, while $\stackrel{\circ}{\mathrm{L}} \stackrel{\circ}{\mathrm{R}}$ and $\stackrel{\circ}{\mathrm{P}}$ stand, resp., for, left , right, and parent.

\begin{tabular}{|c|c|c|c|c|c|c|c|c|c|}
\hline$i$ & $\bar{I}_{r}^{i}(\mathrm{I}, \mathrm{I})$ & $\bar{I}_{r}^{i}(\mathrm{l}, \mathrm{r})$ & $\bar{I}_{r}^{i}(\mathrm{l}, \mathrm{t})$ & $\bar{I}_{r}^{i}(\mathrm{r}, \mathrm{l})$ & $\bar{I}_{r}^{i}(\mathrm{r}, \mathrm{r})$ & $\bar{I}_{r}^{i}(\mathrm{r}, \mathrm{t})$ & $\bar{I}_{r}^{i}(\mathrm{t}, \mathrm{l})$ & $\overline{\bar{I}}_{r}^{i}(\mathrm{t}, \mathrm{r})$ & $\bar{I}_{r}^{i}(\mathrm{t}, \mathrm{t})$ \\
\hline 6 & $\langle\emptyset\rangle$ & false & false & false & $\langle\emptyset\rangle$ & false & false & false & false \\
\hline 7 & $\langle\emptyset\rangle$ & false & false & false & $\langle\emptyset\rangle$ & false & false & false & $\langle\emptyset\rangle$ \\
\hline 8 & $\langle\emptyset\rangle$ & false & false & false & $\langle\emptyset\rangle$ & false & 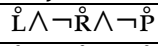 & false & $\langle\emptyset\rangle$ \\
\hline 9 & $\langle\emptyset\rangle$ & false & false & false & $\langle\emptyset\rangle$ & false & 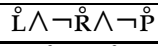 &  & $\langle\emptyset\rangle$ \\
\hline 10 & 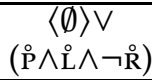 & false & $\stackrel{\circ}{\mathrm{P} \wedge} \wedge \neg \stackrel{\circ}{R}$ & false & $\langle\phi\rangle$ & false & $\stackrel{\circ}{\mathrm{L} \wedge} \neg \stackrel{\circ}{\mathrm{R}}$ & $\stackrel{\circ}{\mathrm{R}} \wedge \neg \stackrel{\circ}{\mathrm{L}} \wedge \neg \stackrel{\circ}{\mathrm{P}}$ & $\begin{array}{c}\langle\emptyset\rangle \vee \\
(\stackrel{\circ}{\mathrm{P}} \wedge \stackrel{\circ}{\mathrm{L}} \wedge \neg \stackrel{\circ}{\mathrm{R}})\end{array}$ \\
\hline 11 & $\langle\emptyset\rangle \vee(\stackrel{\circ}{\mathrm{P}} \wedge \mathrm{L})$ & $\stackrel{\circ}{\mathrm{P}} \wedge \stackrel{\circ}{\mathrm{R}}$ & $\stackrel{\circ}{P}$ & 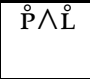 & \begin{tabular}{|c|}
$\langle\emptyset\rangle \vee$ \\
$(\stackrel{\circ}{\mathrm{P}} \wedge \stackrel{\circ}{\mathrm{R}})$
\end{tabular} & $\stackrel{\circ}{P}$ & $\stackrel{\mathrm{L}}{\mathrm{L} \wedge(\stackrel{\circ}{\mathrm{R}} \rightarrow \stackrel{\circ}{\mathrm{P}})}$ & $\stackrel{\circ}{\mathrm{R}} \wedge(\stackrel{\circ}{\mathrm{L}} \rightarrow \stackrel{\circ}{\mathrm{P}})$ & $\begin{array}{c}\langle\emptyset\rangle \vee \\
(\stackrel{\circ}{\mathrm{P}} \wedge(\stackrel{\circ}{\mathrm{L}} \vee \stackrel{\circ}{R}))\end{array}$ \\
\hline
\end{tabular}

\begin{tabular}{|c|c|c|c|}
\hline$i$ & $\bar{I}_{c}^{i}(\mathrm{l})$ & $\bar{I}_{c}^{i}(\mathrm{r})$ & $\bar{I}_{c}^{i}(\mathrm{t})$ \\
\hline 6 & $\langle\emptyset\rangle$ & $\langle\emptyset\rangle$ & false \\
\hline 7 & $\langle\emptyset\rangle$ & $\langle\emptyset\rangle$ & $\langle\emptyset\rangle$ \\
\hline 8 & $\langle\emptyset\rangle$ & $\langle\emptyset\rangle$ & $\langle\emptyset\rangle$ \\
\hline 9 & $\langle\emptyset\rangle$ & $\langle\emptyset\rangle$ & $\langle\emptyset\rangle$ \\
\hline 10 & $\langle\emptyset\rangle \vee(\stackrel{\circ}{\mathrm{P}} \wedge \stackrel{\circ}{\mathrm{L}} \wedge \neg \stackrel{\circ}{\mathrm{R}})$ & $\langle\emptyset\rangle$ & $\langle\emptyset\rangle \vee(\stackrel{\circ}{\mathrm{P}} \wedge \stackrel{\circ}{\mathrm{L}} \wedge \neg \stackrel{\circ}{\mathrm{R}})$ \\
\hline 11 & $\langle\emptyset\rangle \vee(\stackrel{\circ}{\mathrm{P}} \wedge \mathrm{L})$ & $\langle\emptyset\rangle \vee(\stackrel{\circ}{\mathrm{P}} \wedge \stackrel{\circ}{\mathrm{R}})$ & $\langle\emptyset\rangle \vee(\stackrel{\circ}{\mathrm{P}} \wedge(\stackrel{\circ}{\mathrm{L}} \vee \mathrm{\circ}))$ \\
\hline
\end{tabular}

The tables give an idea of how abstract values are obtained; however, line 11 alone is enough to appreciate the results.

The final value for $\bar{I}_{c}(\mathrm{I})$ indicates that a cycle starting at $\sigma^{f}(\mathrm{I})$ is either an empty path or one involving both $\stackrel{\circ}{\mathrm{P}}$ and $\stackrel{\circ}{\mathrm{L}}$. In fact, to traverse $\stackrel{\circ}{\mathrm{P}}$ and $\stackrel{\mathrm{L}}{\mathrm{i}}$ is needed to reach $\sigma^{f}(\mathrm{t})$ and go back to $\sigma^{f}(\mathrm{I})$. There are also cycles which touch $\sigma^{f}(\mathrm{r})$ and traverse all fields at least once; this is also taken into account since the truth assignment \{left, right, parent\} is still a model of $\bar{I}_{c}(\mathrm{I})$. A similar reasoning holds for $\bar{I}_{c}(r)$.

Finally, $\bar{I}_{c}(\mathrm{t})$ represents all kinds of cycles starting from $\sigma^{f}(\mathrm{t})$, each of them corresponding to a model of the path-formula: (1) an empty one; (2) one that reaches $\sigma^{f}(\mathrm{I})$ at least once, without reaching $\sigma^{f}(r)$; (3) a dual one which only reaches $\sigma^{f}(r)$; and (4) one that reaches both $\sigma^{f}(\mathrm{I})$ and $\sigma^{f}(\mathrm{r})$ at least once.

It is important to point out that it is not possible to precisely describe the heap structure by simply inferring a set of fields which have to be traversed by every cycle [Brockschmidt et al. 2012]. In fact, parent is the only field which is involved in every cycle, but this information alone would not be enough to guarantee that a loop going from a leaf to the root terminates. 


\subsection{Back to the double-linked-list example}

Consider again the code discussed in Section 1.3 The following annotated code shows the deep-sharing information after each line upon reaching the fixpoint.

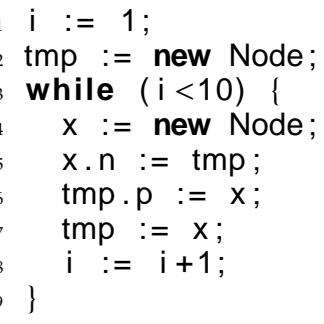

Next table shows how the present analysis works on this example. Again, the first column refers to the program line; primed numbers correspond to the second time a given line is considered; $\bar{I}_{r c}^{i}=\left(\bar{I}_{r}^{i}, \bar{I}_{c}^{i}\right)$ is the abstract value computed after line $i$.

\begin{tabular}{|c|c|c|c|c|c|c|}
\hline$i$ & $\overline{\bar{I}_{r}^{i}(\mathrm{X}, \mathrm{x})}$ & $\overline{\bar{I}_{r}^{i}(\mathrm{x}, \mathrm{tmp})}$ & $\bar{I}_{r}^{i}(\mathrm{tmp}, \mathrm{x})$ & $\bar{I}_{r}^{i}(\mathrm{tmp}, \mathrm{tmp})$ & $\overline{\bar{I}_{c}^{i}(\mathrm{x})}$ & $\overline{\bar{I}_{c}^{i}(\mathrm{tmp})}$ \\
\hline 1 & false & false & false & false & false & false \\
\hline 2,3 & false & false & false & $\langle\emptyset\rangle$ & false & $\langle\phi\rangle$ \\
\hline 4 & $\langle\emptyset\rangle$ & false & false & $\langle\emptyset\rangle$ & $\langle\emptyset\rangle$ & $\langle\emptyset\rangle$ \\
\hline 5 & $\langle\emptyset\rangle$ & $\langle A\rangle$ & false & $\langle\emptyset\rangle$ & $\langle\emptyset\rangle$ & $\langle\emptyset\rangle$ \\
\hline 6 & $\langle\emptyset\rangle \vee\left\langle A \rightarrow, p^{\top}\right\rangle$ & $A$ & $p^{x}$ & $\langle\emptyset\rangle \vee\left\langle A \rightarrow, p^{x}\right\rangle$ & $\langle\emptyset\rangle \vee\left\langle A \geq, p^{x}\right\rangle$ & $\left.\langle\emptyset\rangle \vee\langle A\rangle, p^{x}\right\rangle$ \\
\hline 7,8 & $\langle\emptyset\rangle \vee\left\langle A, p^{\prime}\right\rangle$ & $\langle\emptyset\rangle \vee\left\langle A, p^{\prime}\right\rangle$ & $\langle\emptyset\rangle \vee\left\langle A, p^{x}\right\rangle$ & $\langle\emptyset\rangle \vee\left\langle A, p^{\prime}\right\rangle$ & $\langle\emptyset\rangle \vee\left\langle A^{\prime}, p^{x}\right\rangle$ & $\langle\emptyset\rangle \vee\left\langle A^{\prime}, p^{x}\right\rangle$ \\
\hline $3^{\prime}$ & $\langle\emptyset\rangle \vee\left\langle A, p^{\prime}\right\rangle$ & $\left.\langle\emptyset\rangle \vee\langle A\rangle, p^{\top}\right\rangle$ & $\langle\emptyset\rangle \vee\left\langle A, p^{x}\right\rangle$ & $\langle\emptyset\rangle \vee\left\langle A, p^{\prime}\right\rangle$ & $\langle\emptyset\rangle \vee\left\langle A^{\prime}, p^{x}\right\rangle$ & $\langle\emptyset\rangle \vee\left\langle A^{\prime}, p^{x}\right\rangle$ \\
\hline $4^{\prime}$ & $\langle\emptyset\rangle$ & false & false & $\langle\emptyset\rangle \vee\left\langle A, p^{-}\right\rangle$ & $\langle\emptyset\rangle$ & $\left.\langle\emptyset\rangle \vee\langle A\rangle, p^{-}\right\rangle$ \\
\hline $5^{\prime}$ & $\langle\emptyset\rangle$ & $A$ & false & $\langle\emptyset\rangle \vee\left\langle A \rightarrow, p^{-}\right\rangle$ & $\left.\langle\emptyset\rangle \vee\langle A\rangle, p^{-}\right\rangle$ & $\langle\emptyset\rangle \vee\left\langle A \rightarrow, p^{-}\right\rangle$ \\
\hline $6^{\prime}$ & $\langle\emptyset\rangle \vee\langle A\rangle, p\rangle$ & $A$ & $p^{2}$ & $\langle\emptyset\rangle \vee\left\langle A, p^{\prime}\right\rangle$ & $\left.\langle\emptyset\rangle \vee\langle A\rangle, p^{-}\right\rangle$ & $\left.\langle\emptyset\rangle \vee\langle A\rangle, p^{\top}\right\rangle$ \\
\hline $7^{\prime}, 8$ & $\langle\emptyset\rangle \vee\langle A, p\rangle\rangle$ & $\langle\emptyset\rangle \vee\left\langle A>, p^{\top}\right\rangle$ & $\langle\emptyset\rangle \vee\left\langle A, p^{\prime}\right\rangle$ & $\langle\emptyset\rangle \vee\left\langle A, p^{-}\right\rangle$ & $\langle\emptyset\rangle \vee\langle A>, p\rangle$ & $\left.\langle\emptyset\rangle \vee\langle A\rangle, p^{\top}\right\rangle$ \\
\hline
\end{tabular}

The first reachability information is added at line 2: the creation of an object implies that tmp is reachable from itself and cyclic by means of an empty path. The same happens at line 4 with $x$. The update of $x . n$ implies that tmp is reachable from $x$, and it can be guaranteed that the new path will only traverse $n$. In the following line, a cycle is created by the field update of tmp.p. At this point, both variables are cyclic, and it is possible to guarantee that a cycle will either (1) be empty; or (2) traverse both fields. The value for $\bar{I}_{r}^{6}(\operatorname{tmp}, \mathrm{x})$ is computed as the disjunction between $\bar{I}_{r}^{5}(\mathrm{tmp}, \mathrm{x})=$ false and the new formula $\langle\emptyset\rangle \odot\left(\left\langle p^{\prime}\right\rangle \vee\left(\left\langle p^{\prime}\right\rangle \odot I_{r}^{5}(x, t m p)\right)\right) \odot\langle\emptyset\rangle=p^{\prime}$, giving $p^{\prime}$ as the final result. Note that the disjunction between $\langle p\rangle\rangle$ and $\langle p\rangle\rangle \odot \bar{I}_{r}^{5}(x, t m p)$ takes into account both ways to go from tmp to $x$ : either directly, or traversing the new cycle and touching $x$ twice. Therefore, it is not possible to guarantee that no path will traverse $n$. Line 7 assigns $x$ to tmp, so that the reachability information $I_{r}^{7}(\mathrm{x}, \mathrm{tmp})$ and $I_{r}^{7}(\mathrm{tmp}, \mathrm{x})$ is copied from $I_{r}^{6}(\mathrm{x}, \mathrm{x})$. The second iteration is needed since line 3 is entered the second time with a different abstract value (row $3^{\prime}$ of the table). At row $4^{\prime}$, reachability and cyclicity information about $\mathrm{x}$ is removed, but non-empty cyclicity of tmp is still admitted. A fixpoint is reached after the second iteration, as shown by the fact that row 8 and row $8^{\prime}$ of the table are equal. The final result, which is finally copied to $\bar{I}_{r c}^{9}$, correctly shows that any non-empty cycle reachable from either $\mathrm{x}$ or tmp has to involve both $\mathrm{n}$ and $\mathrm{p}$, so that a loop which only traverses one of these fields (as the one depicted in Section 1.3, lines 10-12) is guaranteed to terminate. 


\section{PRACTICAL ISSUES}

The analysis has been partially implemented 10 in its intra-procedural part, based on the Chord Java bytecode analyzer [Naik 2011]. The implementation covers most sequential Java bytecode instructions which may occur in the single method the analysis focuses on. The examples of Sections 1.2 and 1.3 have been analyzed, and the result appears in the tables of Sections 4.6 and 4.7

A path-formula is explicitly represented as the set of its models, which are, in turn, sets of fields: for example, $\left\{\left\{f l d_{1}\right\},\left\{f l d_{2}\right\}\right\}$ represents the exclusive disjunction $\left(f l d_{1} \wedge \neg f l d_{2}\right) \vee$ $\left(\mathrm{fld}_{2} \wedge \neg \mathrm{fld}_{1}\right)$ if $\mathrm{fld}_{1}$ and $\mathrm{fld}_{2}$ are the only fields in the program. During the fixpoint computation, path-formulæ are always combined by disjunction, which amounts to add new sets of fields (models) to their representation.

Example 5.1 ((implementation of $\odot))$. Consider the field update $v$.fld: $=v^{\prime}$. Let $F_{1}$ and $F_{2}$ be the reachability information from some $w_{1}$ to $v$ and from $v^{\prime}$ to some $w_{2}$, respectively. Both formulæ are represented as sets of sets of fields:

$$
F_{i}=\left\{\left\{f l d_{11}^{i}, . ., f l d_{1 k_{1}}^{i}\right\}, . .,\left\{f l d_{n 1}^{i}, . . ., f l d_{1 k_{n}}^{i}\right\}\right\}
$$

where each truth assignment $\left\{f l d_{j 1}^{i}, . ., f l d_{j k_{j}}^{i}\right\}$ is a model of $F_{i}$. The newly-computed formula

$$
\bar{I}_{r}^{\prime \prime}\left(w_{1}, w_{2}\right)=\bar{I}_{r}^{\prime}\left(w_{1}, v\right) \odot\left(\langle\text { flet }\rangle \vee\left(\langle\text { flet }\rangle \odot \bar{I}_{r}^{\prime}\left(v^{\prime}, v\right)\right)\right) \odot \bar{I}_{r}^{\prime}\left(v^{\prime}, w_{2}\right)
$$

can be obtained by taking each pair of sets $\left(X_{1}, X_{2}\right)$ belonging to $F_{1} \times F_{2}$, and each $Y$ belonging to (the set representation of) $G=\bar{I}_{r}^{\prime}\left(v^{\prime}, v\right)$, and computing the unions $X_{1} \cup\{$ fld $\} \cup X_{2}$ and $X_{1} \cup\{f l d\} \cup Y \cup X_{2}$. More formally,

$$
\bar{I}_{r}^{\prime \prime}\left(w_{1}, w_{2}\right)=\left\{X_{1} \cup\{f l d\} \cup X_{2} \mid X_{i} \in F_{i}\right\} \cup\left\{X_{1} \cup\{\text { fld }\} \cup Y \cup X_{2} \mid X_{i} \in F_{i}, Y \in G\right\}
$$

The global fixpoint terminates when no new models are added to path-formulæ.

\subsection{Scalability}

With respect to similar analyses discussed as related work, the main threats to scalability seem to be the complexity of operations on path-formulæ and the potentially large number of reference fields in a program. The implementation deals with path-formulæ operations such as $\odot$ in the way suggested in Example 5.1. A set of fields can be easily represented as a bit vector, so that union between sets of fields can be efficiently obtained by standard bitwise operations. A large number of fields would imply the need of more memory to store bit vectors, but no significant slowdown in bitwise computations.

Moreover, to have a large number of fields to be tracked does not necessarily mean that operations like the one described in the example above have to deal with a huge number of field sets $X_{i}$ and $Y$ : there is no reason to have path-formulæ with more models if the program has, globally, more fields. Suppose, for example, that the code presented in Section 1.3 is part of a bigger program with many class declarations: the path-formula computed, say, for $\bar{I}_{c}^{8}(\operatorname{tmp})=\langle\emptyset\rangle \vee\left(n>\wedge p^{\prime}\right)$ would have the same two models \{\} and $\{n, p\}$, the difference being that the bit vectors representing them would be much longer vectors with almost all bits set to 0 .

However, there are at least two ways to go in the direction of improving efficiency; both are based on the observation that the analysis of data structures in the heap can benefit from knowledge of what the information to be inferred will be used for. In the case of proving termination of loops traversing such data structures, which is one of the main indirect goals of the present paper, to know in advance which loops will possibly traverse the data structure under study is a valuable piece of information which can significantly improve

$\overline{{ }^{10} \text { Available at http://costa.ls.fi.upm.es/ damiano/reachCycle/. }}$ 
efficiency. The next two paragraphs discuss two improvements relying on the example of Section 1.2. the goal is to gather information on the structure of the tree built by $x:=$ join (tree1, tree2)

knowing in advance that it will be traversed by the loop

while $(x !=$ null $) x:=x$. left

and that the final goal is to prove termination of such a loop.

Backward program slicing. In order to reduce the portion of code to be analyzed, backward static slicing [Xu et al. 2005] can be used. A slice can be computed backwards from the value of $x$ at the program point before the loop, which includes the part of the program potentially affecting the value of the variable $x$ at that point. Backward static slicing is a well-known technique which has been applied to programming languages like Java and has reached a considerable level of maturity.

Field abstraction. The problem of dealing with a large number of reference fields can be alleviated by observing that some fields are not really relevant when focusing on specific parts of the code. Consider the example presented above: if it is known in advance that the only loop traversing the tree pointed to by $\mathrm{x}$ will do it on the left field, then the only question to be answered is: "is $\{$ left $\}$ a model of $\bar{I}_{c}(\mathrm{x})$ ?"; in other words, is there the possibility of a cycle which only traverses left ? This question can be given an answer without knowing exactly which fields are involved in cycles and under which circumstances, so that a simplification can be performed on the implementation of the analysis.

If left is the only field to be tracked explicitly, as in this case, then all the other fields can be abstracted to a special field any, so that the analysis only has to take left and any into account instead of all reference fields in the program. Operations on field sets are also simplified: e.g., $\left\{a n y, f l d_{1}\right\} \cup\left\{a n y, f l d_{2}\right\}=\left\{a n y, f l d_{1}, f l d_{2}\right\}$ where $f l d_{1}$ and $f l d_{2}$ are the fields to be tracked explicitly. On the other hand, $\left\{a n y, f l d_{1}\right\} \backslash\left\{f l d_{2}\right\}$ results in both $\left\{a n y, f l d_{1}\right\}$ and $\left\{f l d_{1}\right\}$ since any could be representing, at that point, exactly fld ${ }_{2}$ alone. In the example above, the models of $\bar{I}_{c}(\mathrm{x})$ come to be \{\} , \{any\}, and \{any, left $\}$, thus making it possible to detect that no cycle will only traverse left. This feature has been added to the implementation described in this section: it is possible to specify manually which fields have to be tracked explicitly; if not specified, then all fields are tracked.

\section{CONCLUSIONS}

The present paper describes a novel approach to cyclicity analysis which is able to provide, even for possibly cyclic data structures, information that is useful to prove termination or bounds on the resource usage of programs traversing them. This is accomplished by considering the fields through which a cyclic path goes through. If, for example, it is possible to prove that any cycle has to involve certain fields, then the result of the analysis can be successfully used to prove the termination of a loop which never traverses any cycle completely.

A typical example is a tree with edges to parent nodes: this is clearly a cyclic data structure, but loops traversing it one-way (e.g., from the root to a leaf, or the other way around) terminate. Existing cyclicity analyses cannot give enough information about this example, so that termination of loops which traverse the data structure cannot be guaranteed. On the contrary, this analysis provides the required field-sensitive information in form of propositional formulæ, which are expressive enough to capture relevant properties of cycles.

Future work will be mainly devoted to complete the prototypical implementation discussed in Section 5. This will involve adding more features like static fields, making the analysis interprocedural, and build a user interface. Moreover, the precision of the abstract semantics and the implementation could be improved, especially on method calls, by a 
field-sensitive version of the deep-sharing analysis of Section 4.1.1. More speculative work will look for further insight into the abstract domains mentioned in Section 3.6.6.

\section{A. PROOFS}

Lemмa 3.7 The viability of a truth assignment $\omega$ is decidable.

Proor. The goal is to find a state where there is a path $\pi$ which traverses all and only the fields whose f-proposition belongs to $\omega$, i.e., $\pi$ should traverse all and only the fields in $\varphi=\{$ fld $\mid$ flet $\in \omega\}$.

The first step is to compute, for every class $\kappa \in \mathcal{K}$, the set of types of objects which can be reached from objects of type $\kappa$ by only traversing fields in $\varphi$, i.e., the reflexive and transitive closure $R^{*}$ of the relation

$$
R=\left\{\left(\kappa^{\prime}, \kappa^{\prime \prime}\right) \mid \kappa^{\prime} \in \mathcal{K} \wedge \exists \kappa^{\prime} . \text { fld } \in \varphi . \mathcal{K}^{\prime} . \text { fld has declared type } \kappa \text {, with } \kappa^{\prime \prime} \leq \kappa\right\}
$$

Note that paths of length 0 are also considered. The closure is computable since $\mathcal{K} \times \mathcal{K}$ is finite and $R^{*} \subseteq \mathcal{K} \times \mathcal{K}$, so it is guaranteed that a fixpoint will be reached in a finite number of steps. In the end, $\left(\mathcal{K}^{\prime}, \mathcal{K}^{\prime \prime}\right) \in R^{*}$ means that it is possible to reach a $\mathcal{K}^{\prime \prime}$ object from a $\kappa^{\prime}$ object in zero or more steps, by only traversing $\varphi$.

The second step is to consider, one at a time, all the permutations of $\varphi$ (which, as a subset of $\mathcal{F}$, is finite). For each permutation $\left\langle\kappa_{p_{1}} . f l d_{p_{1}}, . ., \kappa_{p_{n}} . f l d_{p_{n}}\right\rangle$, a path $\pi$ is searched for, which has the following form: it traverses $\kappa_{p_{1}}$ fld $d_{p_{1}}$, then goes through $\varphi$ from the second location to a location where an object of class $\kappa_{p_{2}}$ is stored, then traverses $\kappa_{p_{2}}$. fld $d_{p_{2}}$, then goes through $\varphi$ from the last-obtained location to a location where an object of class $\kappa_{p_{3}}$ is stored, and so on until it traverses $\kappa_{p_{n}} f f_{p_{n}}$. Such a path would have the desired property of traversing all and only the fields in $\varphi$, regardless of how many times every single field is traversed.

The existence of $\pi$ depends on $R^{*}$. Let $\ell_{i}^{\prime}$ and $\ell_{i}^{\prime \prime}$ be, resp., the locations where the objects before and after traversing $\kappa_{p_{i}}$. fld $d_{p_{i}}$ are stored; the object stored at $\ell_{i}^{\prime}$ would have type $\kappa_{p_{i}}$, while the object stored at $\ell_{i}^{\prime \prime}$ would have some type compatible with class declarations. The possibility to connect some $\ell_{i}^{\prime \prime}$ to $\ell_{i+1}^{\prime}$ (i.e., to fill the gap between consecutive fields in the permutation) depends on whether there is some $\left(\kappa_{i}^{?}, \kappa_{p_{i+1}}\right) \in R^{*}$ s.t. $\kappa_{i}^{?}$ is a subclass of the declared type of $\kappa_{p_{i}} f l d_{p_{i}}$. If such a pair exists, then a heap can be picked where the object at $\ell_{i}^{\prime \prime}$ has type $\kappa_{i}^{\text {? }}$.

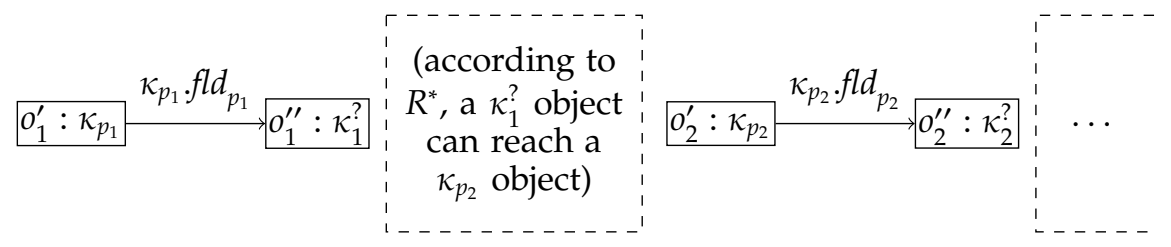

If there is a permutation of $\varphi$ such that all the gaps can be filled, then such a $\pi$ exists, which only traverses fields in $\varphi$, and traverses all of them; in this case, $\omega$ is guaranteed to be viable. On the other hand, if all the permutations have been considered but it was not possible to build $\pi$ for any of them, then $\omega$ is not viable. It is easy to see that the whole process is computable.

Lemma 3.10, The equivalence of two path-formulæ is decidable.

Proof. Truth assignments are finite since $\mathcal{F}$ is also finite. Therefore, it is enough to find a viable one which is a model of one path-formula and a counter-model of the other. To decide if a truth assignment is a model of a formula is easy; moreover, its viability is decidable by Lemma 3.7, so that the whole problem is decidable. 
Lemma A.1. (This lemma is only presented in the appendix) $\bar{\alpha}_{r}$ and $\bar{\gamma}_{r}$ are monotone. Moreover, for every $\bar{I}_{r}^{\prime}, \bar{I}_{r}^{\prime \prime} \in \bar{I}_{r}, \bar{I}_{r}^{\prime} \neq \bar{I}_{r}^{\prime \prime}$ implies $\Rightarrow \bar{\gamma}_{r}\left(\bar{I}_{r}^{\prime}\right) \neq \bar{\gamma}_{r}\left(\bar{I}_{r}^{\prime \prime}\right)$.

Proof. Suppose that there exists a pair of variables $(v, w)$ such that $\bar{I}_{r}^{\prime}(v, w) \not \equiv \bar{I}_{r}^{\prime \prime}(v, w)$; this means that the corresponding path-formulæ do not belong to the same equivalence class, i.e., that there is a viable $\omega$ which is a model of one and only one of them. Suppose $\omega$ is a model of $\bar{I}_{r}^{\prime}(v, w)$ but not of $\bar{I}_{r}^{\prime \prime}(v, w)$ (the dual case is similar). By the definition of the equivalence on $\mathcal{P F}_{\equiv^{v, t}, v}$, there must be a state $\sigma$ where there is a path $\pi$ from $v$ to $w$ traversing all and only the fields belonging to $\left\{f l d \mid f l t^{\prime} \in \omega\right\}$, so that $\pi \rightarrow \bar{I}_{r}^{\prime}(v, w)$, but $\pi \rightarrow \bar{I}_{r}^{\prime \prime}(v, w)$. Obviously, there can be many such states; w.l.o.g., $\sigma$ can be chosen among them, such that $\pi$ is the only path in the heap (note that this is always possible since the other variables are not relevant, and there is no need for any other path from $v$ to $w$ ). Now, $\sigma$ clearly belongs to $\bar{\gamma}_{r}\left(\bar{I}_{r}^{\prime}\right)$, but not to $\bar{\gamma}_{r}\left(\bar{I}_{r}^{\prime \prime}\right)$ (because there is no path-formula $F \leq \bar{I}_{r}^{\prime \prime}(v, w)$ such that $v F$-reaches $w$ in $\sigma$ ), and this concludes the proof.

Lemma $3.16 \bar{\alpha}_{r}$ and $\bar{\gamma}_{r}$ define a Galois insertion between $\overline{\mathcal{I}}_{r}$ and $\mathcal{I}_{b}^{\tau}$.

Proof. We first prove $I_{b} \subseteq \bar{\gamma}_{r}\left(\bar{\alpha}_{r}\left(I_{b}\right)\right)$, i.e., that $\sigma \in I_{b}$ implies $\sigma \in \bar{\gamma}_{r}\left(\bar{\alpha}_{r}\left(I_{b}\right)\right)$. Given a concrete state $\sigma \in I_{b}$, the following holds for every $v$ and $w$ :

$$
G_{v, w}=\bigwedge\{F \mid v F \text {-reaches } w \text { in } \sigma\} \leq\left(\bar{\alpha}_{r}\left(I_{b}\right)\right)(v, w)
$$

since a part of a disjunction always implies the disjunction itself. Now, due to the behavior of path-formulæ, $v G_{v, w}$-reaches $w$ in $\sigma$, so that $G_{v, w}$ is the path-formula $F$ required by $\bar{\gamma}_{r}$ in order to guarantee that $\sigma \in \bar{\gamma}_{r}\left(\bar{\alpha}_{r}\left(I_{b}\right)\right)$.

The second part of the proof demonstrates that $\bar{\alpha}_{r}\left(\bar{\gamma}_{r}\left(\bar{I}_{r}\right)\right) \underline{\Xi}_{r} \bar{I}_{r}$ holds for every $\bar{I}_{r}$. This amounts to saying that

$$
\forall \bar{I}_{r} \cdot \forall v, w \cdot\left(\bar{\alpha}_{r}\left(\bar{\gamma}_{r}\left(\bar{I}_{r}\right)\right)\right)(v, w) \leq \bar{I}_{r}(v, w)
$$

The goal is to prove that any path $\pi$ p-satisfying $\left(\bar{\alpha}_{r}\left(\bar{\gamma}_{r}\left(\bar{I}_{r}\right)\right)\right)(v, w)$ will also p-satisfy $\bar{I}_{r}(v, w)$, since $\leq$ is logical implication. By the definition of $\bar{\alpha}_{r}$ and $\bar{\gamma}_{r}$ :

$$
\begin{aligned}
& \pi \rightarrow\left(\bar{\alpha}_{r}\left(\bar{\gamma}_{r}\left(\bar{I}_{r}\right)\right)\right)(v, w) \\
\Leftrightarrow & \pi \rightarrow \bigvee\left\{\bigwedge\{F \mid v F \text {-reaches } w \text { in } \sigma\} \mid \sigma \in \bar{\gamma}_{r}\left(\bar{I}_{r}\right)\right\} \\
\Leftrightarrow & \pi \rightarrow \bigvee\{\bigwedge\{F \mid v \text {-reaches } w \text { in } \sigma\} \mid \\
& \left.\sigma \in \Sigma_{\tau} \wedge \forall v^{\prime}, w^{\prime} . \exists G \leq \bar{I}_{r}\left(v^{\prime}, w^{\prime}\right) . v^{\prime} G \text {-reaches } w^{\prime} \text { in } \sigma\right\}
\end{aligned}
$$

For every $\sigma$ satisfying $\left(\forall v^{\prime}, w^{\prime} . \exists G \leq \bar{I}_{r}\left(v^{\prime}, w^{\prime}\right) . v^{\prime} G\right.$-reaches $w^{\prime}$ in $\left.\sigma\right)$, the instance $(\exists G \leq$ $\bar{I}_{r}(v, w) . v$ G-reaches $w$ in $\sigma$ ) also holds, where $v$ and $w$ are the variables of interest. This implies $\bigwedge\{F \mid v F$-reaches $w$ in $\sigma\} \leq \bar{I}_{r}(v, w)$ since the mentioned $G$ is actually one of the $F^{\prime}$ s in the conjunction, so that $\bigwedge\{F \mid v F$-reaches $w$ in $\sigma\} \leq G$, and $G \leq \bar{I}_{r}(v, w)$ by hypothesis. Since this holds for every $\sigma$ satisfying the property above (i.e., for all $\sigma \in \bar{\gamma}_{r}\left(\bar{I}_{r}\right)$ ), the disjunction on all such states required by $\bar{\alpha}_{r}$ is still less than or equal to $\bar{I}_{r}(v, w)$. Therefore, since a path-formula

$\bigvee\{\wedge\{F \mid v F$-reaches $w$ in $\sigma\} \mid \sigma$ satisfies the condition above $\}$

has been found such that $\pi$ p-satisfies it, and is less than or equal to $\bar{I}_{r}(v, w)$, the statement $\pi \rightarrow \bar{I}_{r}(v, w)$ holds. Thus, the implication $\pi \rightarrow\left(\bar{\alpha}_{r}\left(\bar{\gamma}_{r}\left(\bar{I}_{r}\right)\right)\right)(v, w) \Rightarrow \pi \rightarrow \bar{I}_{r}(v, w)$ has been proved, and this completes the second part of the proof.

Joining both parts demonstrates that $\bar{\alpha}_{r}$ and $\bar{\gamma}_{r}$ build a Galois connection. Lemma A.1 guarantees that the correspondence is indeed a Galois insertion.

Lemma $3.20 \bar{\alpha}_{c}$ and $\bar{\gamma}_{c}$ define a Galois insertion between $\overline{\mathcal{I}}_{c}$ and $\mathcal{I}_{b}^{\tau}$.

Proof. Very similar to Lemma 3.16. Note that this proof requires to prove monotonicity (easy), and a unicity result for cyclicity similar to Lemma A.1, i.e., that $\bar{I}_{c}^{\prime} \neq \bar{I}_{c}^{\prime \prime}$ implies $\bar{\gamma}_{c}\left(\bar{I}_{c}^{\prime}\right) \neq \bar{\gamma}_{c}\left(\bar{I}_{c}^{\prime \prime}\right)$; such a result is also easy to prove. 
Lemma 3.24. The lattice based on $\left\{\left(\bar{I}_{r}, \bar{I}_{c}\right) \mid \bar{I}_{r} \in \overline{\mathcal{R}}, \bar{I}_{c} \in \bar{Y},\left(\bar{I}_{r}, \bar{I}_{c}\right)\right.$ is in normal form $\}$, with concretization function $\bar{\gamma}_{r c}\left(\bar{I}_{r}, \bar{I}_{c}\right)=\bar{\gamma}_{r}\left(\bar{I}_{r}\right) \cap \bar{\gamma}_{c}\left(\bar{I}_{c}\right)$, is the reduced product of $\overline{\mathcal{I}}_{r}$ and $\overline{\mathcal{I}}_{c}$.

Proof. The goal is to prove that $\bar{\gamma}_{r c}$ is injective, i.e., that $\bar{I}_{r c}^{\prime} \neq \bar{I}_{r c}^{\prime \prime}$ implies $\bar{\gamma}_{r c}\left(\bar{I}_{r c}^{\prime}\right) \neq \bar{\gamma}_{r c}\left(\bar{I}_{r c}^{\prime \prime}\right)$. If $\bar{I}_{r c}^{\prime} \neq \bar{I}_{r c}^{\prime \prime}$, then either (a) $\bar{I}_{r}^{\prime} \neq \bar{I}_{r}^{\prime \prime}$ or (b) $\bar{I}_{c}^{\prime} \neq \bar{I}_{c}^{\prime \prime}$.

(a) Suppose the abstract values differ on the reachability from $v$ to $w$. By Lemma A.1, $\bar{\gamma}_{r}\left(\bar{I}_{r}^{\prime}\right)$ must be different from $\bar{\gamma}_{r}\left(\bar{I}_{r}^{\prime \prime}\right)$. Suppose there are states $\sigma \in \bar{\gamma}_{r}\left(\bar{I}_{r}^{\prime}\right) \backslash \bar{\gamma}_{r}\left(\bar{I}_{r}^{\prime \prime}\right)$ (the dual case is similar). Without loss of generality, $\sigma$ can be taken as follows:

- its behavior on $(v, w)$ is as required by Lemma A.1 there is a path from $v$ to $w$ which p-satisfies $\bar{I}_{r}^{\prime}(v, w)$ but not $\bar{I}_{r}^{\prime \prime}(v, w)$;

- no other variables reach each other;

- if $v$ and $w$ are different variables, then there are no cyclic variables;

- if $v$ and $w$ are the same variable, then the path from $v$ to $w$ is actually a cyclic path, and it is the only cycle in the heap (note that this is allowed since $\bar{I}_{c}^{\prime}(v) \geq \bar{I}_{r}^{\prime}(v, v)$ is required in normal forms).

With this definition, $\sigma \in \bar{\gamma}_{c}\left(\bar{I}_{c}^{\prime}\right)$ and $\sigma \in \bar{\gamma}_{r}\left(\bar{I}_{r}^{\prime}\right)$, so that it belongs to $\bar{\gamma}_{r c}\left(\bar{I}_{r c}^{\prime}\right)$. On the other hand, $\sigma \notin \bar{\gamma}_{r c}\left(\bar{I}_{r c}^{\prime \prime}\right)$ because it does not belong to $\bar{\gamma}_{r}\left(\bar{I}_{r}^{\prime \prime}\right)$.

(b) Suppose the abstract values differ on the cyclicity of $v$. There are results for cyclicity which are similar to their reachability counterpart, and imply $\bar{\gamma}_{c}\left(\bar{I}_{c}^{\prime}\right) \neq \bar{\gamma}_{c}\left(\bar{I}_{c}^{\prime \prime}\right)$. A state $\sigma$ belonging to the set difference $\bar{\gamma}_{c}\left(\bar{I}_{c}^{\prime}\right) \backslash \bar{\gamma}_{c}\left(\bar{I}_{c}^{\prime \prime}\right)$ can be taken as follows:

- its behavior on $v$ is such that there is one and only one cycle on $v$, and such a cycle p-satisfies $\bar{I}_{c}^{\prime}(v)$ but not $\bar{I}_{c}^{\prime \prime}(v)$;

- no variables reach each other, not even $v$ reaches itself (this is possible since the unique cycle on $v$ can be supposed to start from some location reachable from $v$, not from $\sigma^{f}(v)$ itself).

With this definition, $\sigma$ trivially belongs to both $\bar{\gamma}_{r}\left(\bar{I}_{r}^{\prime}\right)$ and $\bar{\gamma}_{r}\left(\bar{I}_{r}^{\prime \prime}\right)$; therefore, it does belong to $\bar{\gamma}_{r c}\left(\bar{I}_{r c}^{\prime}\right)$ although not to $\bar{\gamma}_{r c}\left(\bar{I}_{r c}^{\prime \prime}\right)$.

Lemma 3.26, The abstract domain $\mathcal{I}_{r}^{\tau}$ is an abstraction of $\overline{\mathcal{I}}_{r}$.

Proof. In order to prove this result, an abstraction function $\alpha$ and a concretization function $\gamma$ have to be given, which satisfy the definition of Galois connection. Let $F_{\vee}$ be $\bigvee_{\text {fld } \in \mathcal{F}}$ flet , i.e., the formula having all and only non-empty models.

$$
\begin{aligned}
& \alpha\left(\bar{I}_{r}\right)=\left\{v \rightsquigarrow w \in X^{\rightsquigarrow} \mid \bar{I}_{r}(v, w) \wedge F_{\vee} \neq \text { false }\right\} \\
& \gamma\left(I_{r}\right)=\lambda(v, w) . \begin{cases}\text { true } & \text { if } v \rightsquigarrow w \in I_{r} \\
\neg F_{\vee} & \text { otherwise }\end{cases}
\end{aligned}
$$

$\alpha$ means that $v \leadsto \rightarrow w$ will be included in the abstraction whenever the path-formula $\bar{I}_{r}(v, w)$ has some non-empty model; this is because, unlike $\overline{\mathcal{I}}_{r}, \mathcal{I}_{r}^{\tau}$ only considers paths whose length is at least 1 . On the other hand, $\gamma$ assigns true whenever $v \rightsquigarrow \rightsquigarrow w \in I_{r}$ because $\mathcal{I}_{r}^{\tau}$ does not track conditions on paths; on the other hand, the path-formula returned when $v \leadsto \rightarrow w \notin I_{r}$ still admits paths of length 0 , as expected.

The first thing to prove is that $\gamma\left(\alpha\left(\bar{I}_{r}\right)\right) \bar{\Xi}_{r} \bar{I}_{r}$; this follows easily since (1) if $\bar{I}_{r}(v, w)$ has no non-empty models, then it is either false or equivalent to $\neg F_{\vee}$; therefore, $\left(\gamma\left(\alpha\left(\bar{I}_{r}\right)\right)\right)(v, w)$ is $\neg F_{\vee}$ which is $\geq \bar{I}_{r}(v, w)$. On the other hand, if $(2) \bar{I}_{r}(v, w)$ has non-empty models, then $\left(\gamma\left(\alpha\left(\bar{I}_{r}\right)\right)\right)(v, w)$ is true $\geq \bar{I}_{r}(v, w)$. In both cases, $\left(\gamma\left(\alpha\left(\bar{I}_{r}\right)\right)\right)(v, w) \geq \bar{I}_{r}(v, w)$, which proves the result. Note that, at a first sight, if $\bar{I}_{r}(v, w)=F$ has non-empty models but $v \rightsquigarrow w \notin \notin X^{\rightsquigarrow}$, then the statement will not be added to $\alpha\left(\bar{I}_{r}\right)$, so that $\left(\gamma\left(\alpha\left(\bar{I}_{r}\right)\right)\right)(v, w)$ will be $\neg F_{\vee}$, which seems to be smaller than $F$. However, such case never happens since $v \rightsquigarrow w \notin X^{\rightsquigarrow}$ implies that there is no path from $v$ to $w$ of length $\geq 1$, so that either $F \equiv^{v, w}$ false or $F \equiv^{v, w} \neg F_{\mathrm{V}}$. 
The second part of the proof is to demonstrate that $\alpha\left(\gamma\left(I_{r}\right)\right)$ is smaller than or equal to $I_{r}$. It is straightforward to see that equality holds, so that the correspondence is actually a Galois insertion.

Lemma 3.27 The abstract domain $\overline{\mathcal{I}}_{r}$ is a refinement of the aliasing domain.

Proof. The aliasing domain $\mathcal{I}_{a}$ is defined as the lattice of sets of pairs $\langle v \cdot w\rangle$ of variables, ordered by $\subseteq$, and by the following abstraction and concretization functions w.r.t. the concrete domain:

$$
\begin{aligned}
& \alpha_{a}\left(I_{b}\right)=\left\{\langle v \cdot w\rangle \mid \exists \sigma \in I_{b} \cdot \sigma^{f}(v)=\sigma^{f}(w)\right\} \\
& \gamma_{a}\left(I_{a}\right)=\left\{\sigma \mid \forall v, w \cdot \sigma^{f}(v)=\sigma^{f}(w) \Rightarrow\langle v \cdot w\rangle \in I_{a}\right\}
\end{aligned}
$$

In order to prove the result, the definition of suitable abstraction and concretization functions between $\mathcal{I}_{a}$ and $\overline{\mathcal{I}}_{r}$ is needed.

$$
\begin{aligned}
& \alpha\left(\bar{I}_{r}\right)=\left\{\langle v \cdot w\rangle \mid \bar{I}_{r}(v, w) \wedge\langle\emptyset\rangle \neq \text { false }\right\} \\
& \gamma\left(I_{a}\right)=\lambda v, w \cdot \begin{cases}\text { true } & \text { if }\langle v \cdot w\rangle \in I_{a} \\
\text { false } & \text { otherwise }\end{cases}
\end{aligned}
$$

As usual, it must me proved that $\gamma\left(\alpha\left(\bar{I}_{r}\right)\right) \bar{\beth}_{r} \bar{I}_{r}$ and $\alpha\left(\gamma\left(I_{a}\right)\right)$ is smaller than or equal to $I_{a}$. The first part follows from observing that $\langle v \cdot w\rangle \in \alpha\left(\bar{I}_{r}\right)$ whenever $\emptyset \in \operatorname{models}_{p}\left(\bar{I}_{r}(v, w)\right)$. In this case, $\left(\gamma\left(\alpha\left(\bar{I}_{r}\right)\right)\right)(v, w)$ will be true $\geq \bar{I}_{r}(v, w)$. On the other hand, the equality between $\alpha\left(\gamma\left(I_{a}\right)\right)$ and $I_{a}$ is straightforward.

Lemma $3.29{ }^{k} \mathcal{I}_{r}$ is an abstraction of $\mathcal{I}_{r}^{\tau}$.

Proof. The abstraction and concretization function identifying a Galois insertion between ${ }^{\kappa} \mathcal{I}_{r}$ and $\mathcal{I}_{r}^{\tau}$ are as follows (here, $\delta(v)$ is the declared type of $v$ ):

$$
\begin{aligned}
\alpha\left(I_{r}\right) & =\left\{(\delta(v), \delta(w)) \mid v \leadsto \rightarrow w \in I_{r}\right\} \\
\gamma\left({ }^{(} I_{r}\right) & =\left\{v \leadsto \rightarrow w \mid\left(\kappa_{1}, \kappa_{2}\right) \in{ }^{k} T_{r}, \delta(v) \leq \kappa_{1}, \delta(w) \leq \kappa_{2}\right\}
\end{aligned}
$$

It is straightforward to see that $\gamma\left(\alpha\left(I_{r}\right)\right) \supseteq I_{r}$ since $(1)(\delta(v), \delta(w)) \in \alpha\left(I_{r}\right)$ for every statement $v \rightsquigarrow w \in I_{r} ;(2) v \rightsquigarrow \rightarrow w \in \gamma\left(\alpha\left(I_{r}\right)\right)$ since the declared types of $v$ and $w$ are equal to the $\kappa_{1}$ and $\kappa_{2}$ required in the definition of $\gamma$.

On the other hand, $\alpha\left(\gamma\left({ }^{k} I_{r}\right)\right)={ }^{k} I_{r}$ holds: in fact, applying $\gamma$ adds all statements $v \rightsquigarrow \rightarrow w$ where $v$ and $w$ are any variables with compatible types. Afterward, $\alpha\left(\gamma\left({ }^{\wedge} I_{r}\right)\right)$ is computed as the set of pairs of declared types of all such variables. The last set can be larger, as it could include more subclasses; however, it is equivalent to ${ }^{k} I_{r}$ according to the equivalence relation on $\wp\left(X^{\mathcal{K} \times \mathcal{K}}\right)$.

Lемма 3.31 The following abstraction and concretization functions define a Galois connection between $\overline{\mathcal{I}}_{r}$ and ${ }^{\mathrm{M}} \mathcal{I}_{r}^{\tau}$ : the latter strictly abstracts the former.

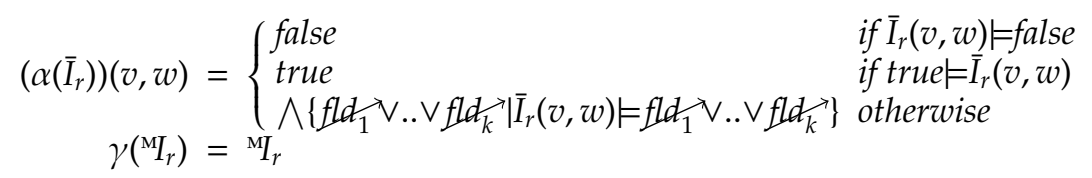

Proof. It is straightforward to see that the path-formula returned by $\alpha$ for every $v$ and $w$ is greater than or equal to $\bar{I}_{r}(v, w)$, so that $\gamma\left(\alpha\left(\bar{I}_{r}\right)\right) \bar{\Xi}_{r} \bar{I}_{r}$.

The second part of the proof, that $\alpha\left(\gamma\left({ }^{\mathrm{M}} I_{r}\right)\right)={ }^{\mathrm{M}} I_{r}$, follows from the fact that ${ }^{\mathrm{M}} I_{r}(v, w)$ is a monotone formula, and, if it is neither true nor false, can be transformed into an equivalent conjunctive normal form: ${ }^{\mathrm{M}} I_{r}[*]=\left(\mathrm{fld}_{11} \vee . . \vee \mathrm{fld}_{\overrightarrow{1 k^{1}}}\right) \wedge . . \wedge\left(\mathrm{fld}_{\overrightarrow{i 1}} \vee . . \vee\right.$ fld $\left._{i k^{i}}\right)$ for some numbers $i$ and $k^{i}$. Each of the $\left(\right.$ fld $_{j j} \vee \vee . \vee$ fld $\left.j k^{j}\right)$ is clearly implied by $\left(\gamma\left({ }^{\mathrm{M}} I_{r}\right)\right)(v, w)$, since the latter is 
logically equivalent to ${ }^{\mathrm{M}} I_{r}[*](v, w)$. Therefore, $\left(\alpha\left(\gamma\left({ }^{\mathrm{M}} I_{r}\right)\right)\right)(v, w)$ is a disjunction of (at least) all the disjunctions (fld $\overrightarrow{i j} \vee . . \vee$ fld $\overrightarrow{j k^{j}}$ ), so that it is less than or equal to (i.e., implies) ${ }^{\mathrm{M}} \mathrm{I}_{r}(v, w)$. If ${ }^{\mathrm{M}} I_{r}(v, w)$ is either true or false, the result is easy.

This proves that there is a Galois insertion between $\overline{\mathcal{I}}_{r}$ and ${ }^{\mathrm{M}} \mathcal{I}_{r}^{\tau}$.

Lemma 3.34. The following functions define a Galois insertion between $\overline{\mathcal{I}}_{r}$ and $\mathrm{A}_{\tau}$ : the latter is a strict abstraction of the former.

$$
\begin{aligned}
& \alpha\left(\bar{I}_{r}\right)=\left\{v \not \sim^{\mathrm{B}} w \mid \forall f l d \in \mathrm{B} . \bar{I}_{r}(v, w) \vDash \neg f l d t\right\} \\
& \gamma\left(I_{A}\right)=\lambda v, w . \bigwedge_{\text {fld } \in \mathrm{B}} \neg \text { flet } \quad \text { where } \mathrm{B} \text { is the maximal set s.t. } v \mu_{\rightarrow}{ }^{\mathrm{B}} w \in I_{A}
\end{aligned}
$$

Proof. The first part demonstrates that $\gamma\left(\alpha\left(\bar{I}_{r}\right)\right) \beth_{r} \bar{I}_{r}$, i.e., that $\bar{I}_{r}(v, w)$ implies $\left(\gamma\left(\alpha\left(\bar{I}_{r}\right)\right)\right)(v, w)$ for every $v$ and $w$. This follows from observing that

$$
\left(\gamma\left(\alpha\left(\bar{I}_{r}\right)\right)\right)(v, w)=\bigwedge\left\{\neg \text { flet } \mid \bar{I}_{r}(v, w) \vDash \neg \text { flet }^{\prime}\right\}
$$

which is clearly implied by $\bar{I}_{r}(v, w)$ since it is a conjunction made of f-propositions which are all implied by $\bar{I}_{r}(v, w)$.

The second part proves that $\alpha\left(\gamma\left(I_{A}\right)\right)=I_{A}$. Let $v \not \rightarrow^{\mathrm{B}} w \in I_{A}$ for some $v$ and $w$. Then, $\left(\gamma\left(I_{A}\right)\right)(v, w)$ will be the conjunction $F$ containing all the negative f-propositions for fields in $\mathrm{B}$ (note that $F$ could contain more literals since $\gamma$ takes the maximal $\mathrm{B}_{0}$ s.t. $v \not \phi_{\rightarrow} \mathrm{B}_{0} w \in I_{A}$ ). It is easy to see that $\alpha\left(\gamma\left(I_{A}\right)\right)$ will contain all statements $v \not x^{\mathrm{B}^{\prime}} w$ such that $\mathrm{B}^{\prime} \subseteq \mathrm{B}_{0}$, thus including $v \quad \mu h \rightarrow \mathrm{B} w$. On the other hand, let $v \quad \mu \rightarrow \mathrm{B} w \in \alpha\left(\gamma\left(I_{A}\right)\right)$ : this means that $\forall$ fld $\in$ B. $\left(\gamma\left(I_{A}\right)\right)(v, w) \vDash \neg f l d$, i.e., that the conjunction $\gamma\left(I_{A}\right)(v, w)$ contains all negated literals $\neg$ flet for fields in $\mathrm{B}$. Therefore, there must be a statement $v \not \boldsymbol{B}^{\prime} \mathrm{B}^{\prime} w \in I_{A}$, with $\mathrm{B} \subseteq \mathrm{B}^{\prime}$. Since abstract values in $A_{\tau}$ are closed under $\subseteq$ [Scapin 2012. Lemma 4.7], $v, \mu \rightarrow^{B} w$ also belongs to $I_{A}$, and the proof is complete.

Lemma 3.36 The following functions define a Galois insertion between $\overline{\mathcal{I}}_{c}$ and $\mathrm{Q}_{\tau}$ : the latter is a strict abstraction of the former.

$$
\begin{aligned}
& \alpha\left(\bar{I}_{c}\right)=I_{Q} \text { with domain } D=\left\{v \mid \bar{I}_{c}(v) \neq \text { false }\right\} \\
& I_{Q}(v)=\left\{f l d \mid \bar{I}_{c}(v) \vDash \text { flet }^{2}\right\} \\
& \gamma\left(I_{Q}\right)=\lambda v \cdot \begin{cases}\bigwedge_{\text {fld } \in I_{Q}(v)} \text { flet } & \text { if } v \in \operatorname{dom}\left(I_{Q}\right) \\
\text { false } & \text { otherwise }\end{cases}
\end{aligned}
$$

Proor. By definition, $\left(\gamma\left(\alpha\left(\bar{I}_{c}\right)\right)\right)(v)$ is false if and only if $\bar{I}_{c}(v)$ was false. On the other hand, if $\bar{I}_{c}(v) \neq$ false, then $\left(\gamma\left(\alpha\left(\bar{I}_{c}\right)\right)\right)(v)$ is the formula $\bigwedge\left\{f \mathrm{fl}^{2} \mid \bar{I}_{c}(v) \vDash\right.$ flet $\}$, which is clearly implied by $\bar{I}_{c}(v)$.

The second part of the proof is also easy: $\alpha\left(\gamma\left(I_{Q}\right)\right)$ is equal to $I_{Q}$ since $v \in \operatorname{dom}\left(\alpha\left(\gamma\left(I_{Q}\right)\right)\right)$ if and only if $\gamma\left(I_{Q}\right) \neq$ false, which holds if and only if $v \in \operatorname{dom}\left(I_{Q}\right)$. Moreover, in this case, $\left(\alpha\left(\gamma\left(I_{Q}\right)\right)\right)(v)$ is exactly the set of fields whose corresponding $\mathrm{f}$-proposition is entailed by $\left(\gamma\left(I_{Q}\right)\right)(v)$. But $\left(\gamma\left(I_{Q}\right)\right)(v)$ comes to be $\bigwedge_{\text {fld } \in I_{Q}(v)} f l l^{\prime}$, and the set of f-propositions entailed by such a formula is exactly $I_{Q}(v)$.

Lemma 4.2. Let $\pi^{\prime}$ and $\pi^{\prime \prime}$ be two paths such that the last location of $\pi^{\prime}$ is the first of $\pi^{\prime \prime}$. Then, $\pi^{\prime} \rightarrow F$ and $\pi^{\prime \prime} \rightarrow G$ imply $\pi^{\prime} \cdot \pi^{\prime \prime} \rightarrow F \odot G$.

Proof. The set of fields traversed by $\pi^{\prime} \cdot \pi^{\prime \prime}$ is the union of the fields traversed by both sub-paths. Since, by hypothesis, the fields traversed by $\pi^{\prime}$ and $\pi^{\prime \prime}$ correspond, respectively, to a model $\omega^{\prime}$ of $F$ and a model $\omega^{\prime \prime}$ of $G$, the union $\omega=\omega^{\prime} \cup \omega^{\prime \prime}$, which is a model of $F \odot G$ 
by definition of $\odot$, is exactly the set of fields traversed by $\pi^{\prime} \cdot \pi^{\prime \prime}$, so that the result clearly holds.

Lemma 4.3. Let $\pi$ be $\pi^{\prime} \cdot \pi^{\prime \prime}$; let $\pi \rightarrow F$ and $\pi^{\prime} \rightarrow G$. Then, $\pi^{\prime \prime} \rightarrow F \ominus G$.

Proor. Let $\varphi$ be the set of fields which are traversed by $\pi$, and $\varphi^{\prime}$ and $\varphi^{\prime \prime}$ be the corresponding field sets for $\pi^{\prime}$ and $\pi^{\prime \prime}$. Clearly, $\varphi=\varphi^{\prime} \cup \varphi^{\prime \prime}$, so that $\varphi^{\prime \prime}$ comes to be the result of removing from $\varphi$ some of the fields belonging to $\varphi^{\prime}$. Such fields are exactly one of the $X$ mentioned in the definition, so that the truth assignment corresponding to $\varphi^{\prime \prime}$ is guaranteed to be a model of $F \ominus G$. Therefore, $\pi^{\prime \prime} \rightarrow F \ominus G$.

Lemma 4.4 Let $\pi$ be $\left\langle\ell_{0}, \ell_{1} . ., \ell_{k}\right\rangle$ and $\pi^{\prime}$ be $\left\langle\ell_{1}, . ., \ell_{k}\right\rangle$. Let the path from $\ell_{0}$ to $\ell_{1}$ traverse fld, and $\pi p$-satisfy F. Then, $\pi^{\prime} \rightarrow F \ominus\langle$ flet $\rangle$.

Proor. If $\pi^{\prime}$ traverses $f l d$ (i.e., this field is traversed at least twice by $\pi$ ), then the set of fields traversed by $\pi^{\prime}$ is the same as $\pi$. Such a set is a model of $F \ominus\left\langle f l t^{\prime}\right\rangle$ since every model of $F$ is also a model of $F \ominus\left\langle f l e^{\prime}\right\rangle$, so that $\pi^{\prime} \rightarrow F \ominus\langle$ flet $\rangle$.

On the other hand, if $\pi^{\prime}$ does not traverse fld, then the set of fields traversed by $\pi^{\prime}$ is a model of $F$ from which fld has been removed. Due to the definition of $\ominus$, such a set is a model of $F \ominus\langle$ flet $\rangle$, so that, again, $\pi^{\prime} \rightarrow F \ominus\left\langle\mathrm{fll}^{2}\right\rangle$.

\section{ACKNOWLEDGMENTS}

This work was funded partially by the European research project FP7-ICT-610582 ENVISAGE: Engineering Virtualized Services (website: http://www.envisage-project.eu), and by the Spanish projects TIN2008-05624 and TIN2012-38137.

\section{REFERENCES}

Albert, E., Arenas, P., Codish, M., Genaim, S., Puebla, G., and Zanardini, D. 2008. Termination analysis of java bytecode. In Int. Conf. on Formal Methods for Open Object-Based Distributed Systems (FMOODS). LNCS, vol. 5051. Springer, 2-18.

Albert, E., Arenas, P., Genaim, S., Puebla, G., and Zanardini, D. 2012. Cost Analysis of Object-Oriented Bytecode Programs. Theoretical Computer Science (Special Issue on Quantitative Aspects of Programming Languages) 413, 1, 142-159.

Albert, E., Genaim, S., And Masud, A.N. 2013. On the inference of resource usage upper and lower bounds. ACM Transactions on Computational Logic 14, 3, 22:1-22:35.

Balaban, I., Pnueli, A., And Zuck, L. D. 2005. Shape Analysis by Predicate Abstraction. In Int. Conf. on Verification, Model Checking, and Abstract Interpretation (VMCAI). LNCS, vol. 3385. Springer, 164-180.

Balaban, I., Pnueli, A., and Zuck, L. D. 2007. Shape Analysis of Single-Parent Heaps. In Int. Conf. on Verification, Model Checking, and Abstract Interpretation (VMCAI). LNCS, vol. 4349. Springer, 91-105.

Bardin, S., Finkel, A., AND NowaK, D. 2004. Toward Symbolic Verification of Programs Handling Pointers. In Int. Workshop on Automated Verification of Infinite-State Systems (AVIS).

Berdine, J., Cook, B., Distefano, D., and O'Hearn, P. 2006. Automatic termination proofs for programs with shape-shifting heaps. In Int. Conf. on Computer Aided Verification (CAV). LNCS, vol. 4144. Springer, 386-400.

Bossi, A., Gabbrielli, M., Levi, G., And Martelli, M. 1994. The s-semantics approach: Theory and applications. Journal of Logic Programming 19E20, 149-197.

Broскsснмidt, M., Musiol, R., Отто, C., and Giesl, J. 2012. Automated Termination Proofs for Java Programs with Cyclic Data. In Int. Conf. on Computer Aided Verification (CAV). LNCS, vol. 7358. Springer, 105-122.

Brotherston, J., Bornat, R., and Calcagno, C. 2008. Cyclic Proofs of Program Termination in Separation Logic. In ACM Symposium on Principles of Programming Languages (POPL). ACM Press, 101-112.

Cook, B., Podelski, A., And Rybalchenko, A. 2006. Termination proofs for systems code. In ACM Conf. on Programming Language Design and Implementation (PLDI). ACM Press, 415-426.

Cousot, P. AND Cousot, R. 1977. Abstract Interpretation: a Unified Lattice Model for Static Analysis of Programs by Construction or Approximation of Fixpoints. In ACM Symposium on Principles of Programming Languages (POPL). ACM Press, 238-252.

Cousot, P. And Cousot, R. 1979. Systematic Design of Program Analysis Frameworks. In ACM Symposium on Principles of Programming Languages (POPL). ACM Press, 269-282. 
Debray, S. K. and Lin, N. W. 1993. Cost analysis of logic programs. ACM Transactions on Programming Languages and Systems 15, 5 (November), 826-875.

Genaim, S. And Spoto, F. 2008. Constancy analysis. In Workshop on Formal Techniques for Java-like Programs.

Genaim, S. and Zanardini, D. 2010. The acyclicity inference of COSTA. In Int. Workshop on Termination.

Genaim, S. and Zanardini, D. 2013. Reachability-based Acyclicity Analysis by Abstract Interpretation. Theoretical Computer Science 474, 0, 60-79.

Ghiya, R. And Hendren, L. J. 1996. Is it a tree, a dag, or a cyclic graph? a shape analysis for heap-directed pointers in c. In ACM Symposium on Principles of Programming Languages (POPL). ACM Press, 1-15.

Giesl, J., Schneider-Kamp, P., and Thiemann, R. 2006. Automatic termination proofs in the dependency pair framework. In Int. Joint Conf. on Automated Reasoning (IJCAR). LNCS (LNAI), vol. 4130. Springer, 281-286.

Gotsman, A., Berdine, J., AND CoOK, B. 2006. Interprocedural shape analysis with separated heap abstractions. In Static Analysis Symposium (SAS). LNCS, vol. 4134. Springer, 240-260.

Graf, S. and Saïd, H. 1997. Construction of abstract state graphs with PVS. In Int. Conf. on Computer Aided Verification (CAV). LNCS, vol. 1254. Springer, 72-83.

Hind, M. 2001. Pointer analysis: haven't we solved this problem yet? In ACM Workshop on Program Analysis For Software Tools and Engineering (PASTE). 54-61.

Jones, R. AND Lins, R. 1996. Garbage collection: algorithms for automatic dynamic memory management. John Wiley \& Sons, Inc., New York, NY, USA.

Kreiker, J., Reps, T., Rinetzky, N., Sagiv, M., Wilhelm, R., and Yahav, E. 2013. Interprocedural Shape Analysis for Effectively Cutpoint-Free Programs. Programming Logics. LNCS. vol. 7797. Springer, 414-445.

Müller-Olm, M., Schmidt, D. A., And Steffen, B. 1999. Model-Checking: A Tutorial Introduction. In Static Analysis Symposium (SAS). LNCS, vol. 1694. Springer, 330-354.

NAIK, M. 2011. Chord: A Versatile Platform for Program Analysis. User Manual.

Nelson, G. 1983. Verifying Reachability Invariants of Linked Structures. In ACM Symposium on Principles of Programming Languages (POPL). ACM Press, 38-47.

Nikolic, D. And Spoto, F. 2014. Reachability analysis of program variables. In ACM Transactions on Programming Languages and Systems 35, 4, . 14:1-14:68.

Reynolds, J. C. 2002. Separation Logic: A Logic for Shared Mutable Data Structures. In IEEE Symposium on Logic in Computer Science (LICS). 55-74.

Rinetzky, N., BAuER, J., Reps, T. W., SAgiv, S., AND Wilhelm, R. 2005. A semantics for procedure local heaps and its abstractions. In ACM Symposium on Principles of Programming Languages (POPL). ACM Press, 296-309.

Rossignoli, S. And Sрото, F. 2006. Detecting Non-Cyclicity by Abstract Compilation into Boolean Functions. In Int. Conf. on Verification, Model Checking, and Abstract Interpretation (VMCAI). LNCS, vol. 3855. Springer, 95-110.

Sagiv, S., Reps, T. W., And Wilhelm, R. 2002. Parametric shape analysis via 3-valued logic. ACM Transactions on Programming Languages and Systems 24, 3, 217-298.

Scapin, E. 2012. Field-Sensitive Unreachability and Non-Cyclicity Analysis. M.S. thesis, Dept. of Computer Science, University of Verona.

Secci, S. and Spoto, F. 2005. Pair-Sharing Analysis of Object-Oriented Programs. In Static Analysis Symposium (SAS). LNCS, vol. 3672. Springer, 320-335.

Spoto, F. And Jensen, T. 2003. Class analyses as abstract interpretations of trace semantics. ACM Transactions on Programming Languages and Systems 25, 5, 578-630.

Spoto, F., Mesnard, F., And Payet, É. 2010. A Termination Analyser for Java Bytecode based on Path-Length. ACM Transactions on Programming Languages and Systems 32, 3. Article 8.

Wegbreit, B. 1975. Mechanical Program Analysis. Communications of the ACM 18, 9, 528-539.

Wilhelm, R., SAgiv, S., ANd RePs, T. W. 2000. Shape analysis. In Int. Conf. on Compiler Construction (CC). LNCS, vol. 1781. Springer, 1-17.

Xu, B., Qian, J., Zhang, X., Wu, Z., and Chen, L. 2005. A Brief Survey of Program Slicing. SIGSOFT Software Engineering Notes 30, 2, 1-36.

Received Month Year; revised Month Year; accepted Month Year 